\title{
Kalman Filter Estimation of Underwater Vehicle Position and Attitude Using a Doppler Velocity Aided Inertial Motion Unit
}

\author{
by \\ Daniel Eugene Leader \\ B.S. in Marine Engineering, United States Naval Academy (1982) \\ Submitted in partial fulfillment of the \\ requirements for the degree of \\ OCEAN ENGINEER \\ at the \\ MASSACHUSETTS INSTITUTE OF TECHNOLOGY \\ and the \\ WOODS HOLE OCEANOGRAPHIC INSTITUTION
}

September 1994

(C) Daniel E. Leader

The author hereby grants to MIT and WHOI permission to reproduce and to distribute copies of this thesis document in whole or in part.

Author . .

Joint Program in Oceanographic Engineering

Massachusetts Institute of Technology/

Woods Hole Oceanographic Institution

12 September 1994

Certified by ...........

Dr. W. Kenneth Stewart, Jr.

Woods Hole Oceanographic Institution

Thesis Supervisor

Accepted by

Professor Arthur B. Baggeroer

Chair, Joint Committee for Oceanographic Engineering Massachusetts Institute of Technology/ Woods Hole Oceanographic Institution 


\title{
Kalman Filter Estimation of Underwater Vehicle Position and Attitude Using a Doppler Velocity Aided Inertial Motion Unit
}

\author{
by \\ Daniel Eugene Leader \\ Submitted to the Massachusetts Institute of Technology and \\ the Woods Hole Oceanographic Institution \\ Joint Program in Oceanographic Engineering \\ in September, 1994, in partial fulfillment of the requirements for the degree of \\ OCEAN ENGINEER
}

\begin{abstract}
This Paper explores the use of an extended Kalman filter to provide real-time estimates of underwater vehicle position and attitude. The types of previously available sensors are detailed including strapdown accelerometers, roll and pitch sensors, gyro and magnetic compasses, depth sensor, and various types of acoustic positioning systems. A doppler velocimeter is added to this sensor suite to improve the performance of the filter. As an integral part of the filter, magnetic compass and gyrocompass biases are estimated to improve vehicle heading accuracy. The filter is designed to account for numerous reallife complications. These include varying rates of sensor output, lengthy gaps in reception of position information, presence of non-Gaussian position fix errors (flyers), and varying probability density functions for sensor errors. Simulated data are used to test the filter with varying availability of data and accuracy of initial conditions, along with actual data from a deployment of the towed DSL-120 vehicle. The increased accuracy obtained by using the doppler velocimeter is emphasized.
\end{abstract}

Thesis Supervisor: Dr. W. Kenneth Stewart, Jr.

Associate Scientist

Woods Hole Oceanographic Institution 


\section{Acknowledgments}

This thesis was completed with the help of many people. At the risk of leaving someone out (for which I ask forgiveness in advance), I would like to mention them:

Dr. W. Kenneth Stewart, Jr., my thesis advisor, who pointed me in the right direction and gave me the guidance I needed to finish this project.

Jon Howland and Marty Marra, who patiently endured my many questions about Deep Submergence Laboratory (DSL) equipment and the computer systems in use there.

My fellow graduate students Diane Di Massa, who offered many good suggestions during numerous chapter rewrites; Hanumant Singh, who unselfishly gave of his time in helping me with the $\mathrm{C}$ computer code needed to translate raw data into usable information; Tad Snow, who served as a sounding board for numerous ideas, both good and bad; and my fellow Navy students Chris Willy and Mike Hajosy, who helped me keep a good perspective through it all.

Dr. Arthur Baggeroer of MIT and the staff of the Woods Hole Oceanographic Institution Education Office for their assistance in handling the administrative requirements of the program.

The staff of the Navy Academic Office at MIT, especially Jennifer Laible, for ably facilitating all liaison with the Navy.

The Unites States Navy for providing this wonderful opportunity.

Finally, my children Christopher and Elizabeth, and most of all my wife Sally, who provided constant encouragement and support, and who always make it all worthwhile. 


\section{Contents}

1 Introduction . . . . . . . . . . . . . . . . 10

1.1 Motivation. . . . . . . . . . . . . . . . 10

1.1.1 Improving Vehicle Position and Attitude Information. . . . . . . . 10

1.1.2 Previous Work on Underwater Vehicle Navigation. . . . . . . . . 11

1.1.3 Cost and Processing Time Reduction. . . . . . . . . . . . . . 12

1.1.4 Resources Available. . . . . . . . . . . . . . . . . . . . . 13

1.2 Advantages and Limitations of the Kalman Filter . . . . . . . . . . . . . 13

1.3 Thesis Outline. . . . . . . . . . . . . . . . . . . . . . . . . 14

2 Sensor Information. . . . . . . . . . . . . . . . . . . 16

2.1 Overview of Sensors. . . . . . . . . . . . . . . . 16

2.2 Coordinate Systems. . . . . . . . . . . . . . . . . . . . 16

2.3 Doppler Velocimeter. . . . . . . . . . . . . . . . . . . . 17

2.3.1 Principles of Operation . . . . . . . . . . . . . . . 18

2.3.2 Inputs to Velocimeter. . . . . . . . . . . . . . . . . . . . 22

2.3.3 Doppler Velocimeter Outputs . . . . . . . . . . . . . . . 23

2.4 Depth Sensor. . . . . . . . . . . . . . . . . . . . . . . . . 24

2.5 External Positioning System . . . . . . . . . . . . . . . . . . . 25

2.5.1 Long-baseline Systems . . . . . . . . . . . . . . . . . . 25

2.5.2 Short-baseline Systems . . . . . . . . . . . . . . . . 27

2.5.3 Ultrashort-baseline Systems . . . . . . . . . . . . . . . . . 27

2.5.4 Other Positioning Systerns . . . . . . . . . . . . . . . . 28

2.6 Gyro. . . . . . . . . . . . . . . . . . . 29

2.7 Flux Gate Magnetic Compass . . . . . . . . . . . . . . . . . . . 29

2.8 Inclinometer. . . . . . . . . . . . . . . . . . . . . 30

2.9 Systron Donner MotionPak (Inertial Motion Unit) . . . . . . . . . . . 31

2.9.1 Accelerometer Operation . . . . . . . . . . . . . . . . . . . 31

2.9.2 Angular Velocity Measurements . . . . . . . . . . . . . . . .32 
3 Development of the Kalman Filter Model. . . . . . . . . . . . . . . . 34

3.1 Characteristics of the Linear Kalman Filter. . . . . . . . . . . . . . 34

3.1.1 System Model. . . . . . . . . . . . . . . . . . . . . . . 35

3.1.2 System Model Dynamics. . . . . . . . . . . . . . . . . 35

3.2 Sensor Outputs and Their Relationship to the State Space . . . . . . . . 37

3.2.1 IMU Package. . . . . . . . . . . . . . . . . . . . . . . . 37

3.2.2 Attitude Package. . . . . . . . . . . . . . . . . . . 38

3.2.3 Doppler Velocimeter Package. . . . . . . . . . . . . . . . . 38

3.2.4 Positioning System Package. . . . . . . . . . . . . . . . .38

3.3 Defining the State Space. . . . . . . . . . . . . . . . . . . 39

3.4 Nonlinearity and the Extended Kalman Filter . . . . . . . . . . . . 40

3.4.1 Choosing the Extended Kalman Filter . . . . . . . . . . . . .41

3.4.2 Filter Propagation. .. . . . . . . . . . . . . . . . . . . .41

3.4.2.1 Update Step. . . . . . . . . . . . . . . . . . 442

3.4.2.2 Prediction Step. . . . . . . . . . . . . . . . . . 43

3.4.3 Implications of the Extended Kalman Filter . . . . . . . . . . . 44

3.5 Modeling System Noise. . . . . . . . . . . . . . . . . . . . . 44

3.5.1 Eliminating Non-Gaussian Position System Errors . . . . . . . . 45

3.5.2 Random Forcing Function Noise . . . . . . . . . . . . . . . .46

3.5.3 Measurement Uncertainty. . . . . . . . . . . . . . . . . .46

3.5.3.1 Linear Accelerometer Uncertainty. . . . . . . . . . . .46

3.5.3.2 Doppler Velocimeter Uncertainty. . . . . . . . . . . . .47

3.5.3.3 Position System Uncertainty. . . . . . . . . . . . . .48

3.5.3.4 Depth Sensor Uncertainty . . . . . . . . . . . . . . 48

3.5.3.5 Angular Velocimeter Uncertainty. . . . . . . . . . . . .49

3.5.3.6 Pitch and Roll Uncertainty. . . . . . . . . . . . . . 49

3.5.3.7 Heading Uncertainty . . . . . . . . . . . . . . . . 49

3.6 Establishing Reference Points and Initial Conditions. . . . . . . . . . 50

3.6.1 Reference Points. . . . . . . . . . . . . . . . . . . . . 50

3.6.2 Initial State Vector and Error Covariance Matrix . . . . . . . . 51

3.6.3 Removing Known Biases. . . . . . . . . . . . . . 51 
3.7 Changes Required to Standard Kalman Filter. . . . . . . . . . . . . . 52

3.7.1 Adjustments Required Due to Varying Filter Update Rate . . . . . 53

3.7.1.1 Determining the Desired Time Step. . . . . . . . . . . 53

3.7.1.2 Predicting the Next State Vector and Error Covariance Matrix 53

3.7.2 Coordinate System Transformations. . . . . . . . . . . . . . 54

3.7.2.1 Removing the Effect of the Gravity Vector . . . . . . . . 54

3.7.2.2 Calculating the $A$ Matrix. . . . . . . . . . . . 55

3.7.3 Varying the $C$ Matrix with the Available Data . . . . . . . 57

3.7.4 Incorporating Fading Memory . . . . . . . . . . . . . . .61

3.8 Observability. . . . . . . . . . . . . . . . . . . . 62

4 Kalman Filter Performance Using Simulated Data . . . . . . . . . .65

4.1 Purpose of Simulation . . . . . . . . . . . . . . . . . . . . 65

4.2 Data Generation. . . . . . . . . . . . . . . . . 65

4.2.1 Method of Data Generation. . . . . . . . . . . . . . . . . 65

4.2.2 Noise-free Data Used for All Simulation Tests. . . . . . . . . 66

4.3 Simulation Tests. . . . . . . . . . . . . . . . . . . . . . . . .69

4.3.1 Test 1: Accurate Initial Conditions . . . . . . . . . . . . . . . .69

4.3.2 Test 2: Inaccurate Initial Conditions Combined with Position Data Gap 72

4.3.3 Test 3: Estimation Without Doppler Velocimeter. . . . . . . . . 73

5 Kalman Filter Performance Using Actual Data. . . . . . . . . . . . . .78

5.1 Description of Data . . . . . . . . . . . . . . . . . . . . . . . .78

5.2 First Data Set. . . . . . . . . . . . . . . . . . . . . . . .78

5.3 Second Data Set . . . . . . . . . . . . . . . . . . . . . . . . .82

6 Conclusions. . . . . . . . . . . . . . . . . 87

6.1 Fulfillment of Objectives. . . . . . . . . . . . . . . . . 87

6.2 Future Work. . . . . . . . . . . . . . . . . . . . . . . . . 88

Bibliography. . . . . . . . . . . . . . . . . . . . 90

Appendix. . . . . . . . . . . . . . . . . . . 92 


\section{List of Figures}

\section{Sensor Information}

2-1 Vehicle-referenced coordinate system . . . . . . . . . . . . . 17

2-2 Broadband ACDP setup for current profiling. . . . . . . . . . . . . 18

2-3 ADCP beam geometry . . . . . . . . . . . . . . . . . . . . . . 19

2-4 Frequency change caused by doppler effect . . . . . . . . . . . 20

2-5 Phase change seen by the broadband system . . . . . . . . . . . 21

2-6 Standard deviation for a single ping using the $150-\mathrm{kHz}$ system . . . . . . 24

2-7 Accelerometer sensor exploded view . . . . . . . . . . . . . . . .31

2-8 Accelerometer system functional diagram . . . . . . . . . . . . . . 32

\section{Development of the Kalman Filter Model}

3-1 System model for $\mathrm{X}$-position propagation. . . . . . . . . . . . . . . 36

3-2 System model for vehicle roll propagation. . . . . . . . . . . . . . 37

3-3 Effect of gravity vector on measured X-acceleration. . . . . . . . . . . 47

3-4 Calculation of true heading and standard deviation. . . . . . . . . 50

3-5 Effect of gravity vector on measured $\mathrm{X}$-acceleration . . . . . . . . . 55

\section{Kalman Filter Performance Using Simulated Data}

4-1 Noise-free measurement of velocity. . . . . . . . . . . . . . . 67

4-2 Noise-free measurement of Y-position. . . . . . . . . . . . . . 67

4-3 Noise-free measurement of vehicle pitch . . . . . . . . . . . .68

4-4 Noise-free measurement of heading by gyro . . . . . . . . . . . 68

4-5 Comparison of measured and estimated $X-Y$ position. . . . . . . . . . 69

4-6 Close-up of $\mathrm{X}-\mathrm{Y}$ position comparison . . . . . . . . . . . . . . 70

4-7 Comparison of actual and estimated $Z$-position . . . . . . . . . . . . 70

4-8 Comparison of actual and estimated roll . . . . . . . . . . . . . .71

4-9 Comparison of actual and estimated heading . . . . . . . . . . . . .71

4-10 Actual and estimated gyro bias . . . . . . . . . . . . . . . . . 72

4-11 Comparison of measured and estimated $\mathrm{X}-\mathrm{Y}$ positions . . . . . . . . . 73 
4-12 Heading estimate . . . . . . . . . . . . . . . . . . 74

4-13 Estimated and actual gyro bias ... . . . . . . . . . . . . 74

4-14 Estimate of magnetic compass bias . . . . . . . . . . . . 75

4-15 Comparison of measured and estimated $X-Y$ position . . . . . . . . . 76

4-16 Estimated heading . . . . . . . . . . . . . . . 76

4-17 Comparison of actual and estimated gyro bias . . . . . . . . . . . 77

4-18 Estimated magnetic compass bias . . . . . . . . . . . . . . . . . 77

\section{Kalman Filter Performance Using Actual Data}

5-1 Output of linear accelerometer . . . . . . . . . . . . . . . 79

5-2 Comparison of estimated and actual $X-Y$ positions. . . . . . . . . . 80

5-3 Close-up of $\mathrm{X}-\mathrm{Y}$ position compurison. . . . . . . . . . . . . . . . . . .80

5-4 Comparison of measured and estimated roll. . . . . . . . . . . . 81

5-5 Estimated heading . . . . . . . . . . . . . . . . . . . . . . . 81

5-6 Estimated compass bias. . . . . . . . . . . . . . . . . . . . .82

5-7 X-Y position estimates compared to position fixes. . . . . . . . . 83

5-8 Comparison of estimated and actual vehicle track. . . . . . . . . . . 84

5-9 Close-up of region of discontinuity following gap in position information . . . 84

$5-10 \mathrm{X}$-position behavior in the vicinity of the discontinuity . . . . . . . 85

5-11 Estimated heading. . . . . . . . . . . . . . . . . . . . . . . 85

5-12 Comparison of measured and estimated Z-position. . . . . . . . . . . 86 


\section{List of Tables}

\section{Sensor Information}

2-1 Comparison of coordinate systems. . . . . . . . . . . . . 17

\section{Kalmán Filter Performance Using Simulated Data}

4-1 Initial conditions for simulations. . . . . . . . . . . . . . 66

4-2 Initial conditions for simulation test 2 . . . . . . . . . . . 72 


\section{Chapter 1}

\section{Introduction}

\subsection{Motivation}

This thesis has two major goals. The first is to provide accurate estimates of underwater vehicle position and attitude and thereby improve the quality of sonar and video information collected. The second is to provide these estimates in real-time.

\subsubsection{Improving Vehicle Position and Attitude Information}

More than two thirds of the Earth is covered by water. Until very recently in history, the ocean depths were mysterious and inaccessible. However, in the past few decades new technologies have dramatically expanded man's abilities to explore the ocean bottom. Discoveries range from the remnants of human incursions, epitomized by the Titanic, to new life on the ocean floor gaining its sustenance from the earth's interior rather than the sun.

One of the primary tools for conducting underwater searches and surveys from unmanned vehicles is the sidescan sonar. The sonar emits a beam of relatively highfrequency sound on either side of the vehicle. When this sound beam hits the bottom or any objects within range, it is reflected and the return is received by transducers mounted on the vehicle. The characteristics of the received sound, primarily magnitude and phase, can then be analyzed to determine the characteristics and contours of the bottom as well as the location and general shape of any objects.

For this system to work at an optimum level, it is vital that the location and attitude of the vehicle be known as accurately as possible. Any position errors lead directly and obviously to an error in the assumed position of all objects mapped by the sonar. Equally important, however, is knowledge of the vehicle's roll, pitch, and heading. Due to the nature of sound propagation in water, a significant time lag occurs between the transmission of a sonar ping and the receipt of any returns. During this time, the vehicle will not only have changed position but may have changed its attitude as well. For proper 
interpretation of the sonar returns, the attitude of the vehicle at the time of sonar transmission must be known, because that determines the precise direction of the beam. This same information is also needed at the time of the sonar return, so the location of any objects found can be accurately determined.

\subsubsection{Previous Work on Underwater Vehicle Navigation}

The invention of feasible inertial navigation produced a great interest in combining inertial measurements with other fix sources to improve navigational accuracy. These early inertial navigation units required gimbal-stabilized platforms to achieve the desired accuracy, which added size and weight. Due to these restrictions, early work was confined to aircraft and ships, including submarines. In the early 1970s, the U.S. Air Force was one of the first to evaluate the use of the Kalman filter to integrate inertial measurements with external fix sources (D'Appolito, 1971).

Later, strapdown inertial systems permitted much smaller and lighter equipment, though at a cost in accuracy. Once again, the military provided a major impetus to developing these systems to their fullest potential. For example, the North Atlantic Treaty Organization's (NATO) Advisory Group for Aerospace Research and Development (AGARD) included much work on these strapdown systems, although still for use primarily in aircraft and spacecraft (VanBronkhorst, 1978; Carford, 1978).

Ever since remotely operated vehicles (ROVs) became feasible, there has been interest in improving their navigational accuracy as well. Early ROVs had only a few sensors available to provide navigational information, since inertial navigational systems were still too heavy and power-intensive to be usable. These included a magnetic compass to provide heading and inclinometers to measure pitch and roll. Additionally, an acoustic transponder network was often used to provide position information for operations in a small area. A later improvement was the gyrocompass, which provides a more consistent output than the magnetic compass but adds new errors caused by drift.

Improvements in the information provided by these acoustic transponder nets has been marked. In her 1992 thesis, Diane Di Massa provided one example with her exploration of the possibilities of hyperbolic navigation to permit long-range acoustic navigation by an Autonomous Underwater Vehicle (AUV) (Di Massa, 1992). Brian 
Tracey contributed another with his improvements to a newer technique for ROV navigation, ultrashort-baseline navigation, in his 1992 thesis (Tracey, 1992).

In the past few years, a number of new devices have been developed to assist in solving this navigational problem. In his 1988 thesis, Gregory Vaughn discussed the use of a lightweight, strapdown inertial motion unit in conjunction with an external acoustic positioning net to improve closed-loop vehicle control (Vaughn, 1988). He concluded that by incorporating this new data into a Kalman filter to provide real-time optimal estimates of vehicle parameters, closed-loop control of ROVs, such as the Woods Hole Oceanographic Institution's Jason, could be improved. However, for his application vehicle attitude was unimportant and therefore was not incorporated into his simulations.

A fairly recent addition to the repertoire of instruments available to the oceanographic engineer is the doppler velocimeter, also known as the acoustic doppler current profiler (ADCP). For the first time, accurate real-time velocity measurements of a ROV could be made relatively cheaply and without exceeding the restrictive power and weight limits inherent in ROV operations. RD Instruments, a doppler velocimeter manufacturer, has been funded by the Otfice of Naval Research to explore the use of the instrument to provide an independent and accurate measurement of vehicle velocity within the context of a Kalman filter. Their initial work verifies the utility of the velocimeter, although so far their research has been confined to inertial navigation systems too large for ROV use (Rowe and Brumley, 1992).

These are but a few examples of the extensive work that has been published on improving underwater vehicle navigation. A search of the literature reveals diverse techniques for improving sensor information and integration, including several variations on Kalman filtering techniques.

\subsubsection{Cost and Processing Time Reduction}

In the real world, cost considerations are almost always important. Cruises are expensive and usually result in computer disks full of unprocessed, raw data, which are essentially worthless until processed. This processing can take many man hours, which translates into a considerable cost. Unfortunately, this sometimes means that procéssing is never completed, since either the time or the money runs out. Therefore, the more real- 
time processing that can be performed the better. Real-time processing means that the important data are immediately available. Also, since it is accomplished at sea while the cruise is in progress (and already paid for), additional expenses are minimized.

Another important application for this capability pertains to autonomous underwater vehicles (AUVs). Much research is being conducted currently on these vehicles, which should be capable of operating independently for days or even weeks. Real-time processing is essential to permit the vehicle to navigate successfully and is also vital in reducing the on-board data storage requirements of AUV deployments.

\subsubsection{Resources Available}

This thesis investigates how the navigational sensors currently available at the Deep Submergence Laboratory (DSL) can be used to improve the real-time position and attitude information available for the operation of a ROV. This summer, DSL personnel participated in a cruise aboard the R/V Knorr: A major purpose of the cruise was to obtain detailed sidescan sonar data from an area of the Mid-Atlantic Ridge where seafloor spreading is occurring. The DSL-120, a towed vehicle carrying a $120-\mathrm{kHz}$ sidescan sonar system and multiple navigational sensors, was used for this survey. Data from these DSL120 deployments are used to demonstrate the performance of the Kalman filter developed in this thesis. By integrating the velocity data provided by the doppler velocimeter into a Kalman filter algorithm, the vehicle's navigational accuracy can be greatly enhanced. This substantially improves the usefulness of the sonar data collected and demonstrates the feasibility of performing real-time navigation sensor processing.

\subsection{Advantages and Limitations of the Kalman Filter}

Sonar data collected by underwater vehicles has often been rendered nearly useless due to inaccurate navigation information and an overly simplistic approach to tracking vehicle parameters. For example, relying solely on a magnetic compass for heading information nearly guarantees that the measured heading will be inaccurate.

The Kalman filter uses a state space model to provide a well defined method for integrating the information obtained from different sensors into a coherent whole. Taking 
advantage of the a priori knowledge of the relative accuracies of these sensors as well as the dynamics of the system, the Kalman filter can provide real-time output without overstressing computer memory or computational limits.

The addition of the doppler velocimeter provides a marked improvement in the ability of a Kalman filter to provide valid position and attitude information. By providing accurate measurements of the magnitude of vehicle velocities in all three dimensions, a more precise, continuous estimate of velicle position and attitude can be provided than would be available by the other sensors alone.

In this application, there are several obstacles to overcome in creating a usable Kalman filter. First, the system is nonlinear and cannot therefore use the simplest and most mathematically precise version of Kalman filter theory. Instead, an extended Kalman filter is required. Second, a method for ignoring obviously faulty measurements must be included. Finally, there are several practical problems involved in using the output of such a wide variety of sensors. These problems and their proposed solutions are discussed in detail later in this thesis.

\subsection{Thesis Outline}

Chapter Two describes the sensors used to provide information for the Kalman filter. Principles of operation for each sensor are discussed, as well as limitations and considerations for each when integrated into the overall system.

Chapter Three explains the development of the Kalman filter and its state space model for this application. A basic review of Kalman filter theory is included, with emphasis on the specifics of the extended Kalman filter required for nonlinear problems. This chapter also discusses the specific problems that were addressed for proper filter operation and how the filter was implemented.

Chapter Four shows the results of filter operation by using both simulated and actual data. The improvement in performance obtained by using the doppler velocimeter measurements of vehicle velocities is emphasized. 
The results are summarized in Chapter Five, along with suggestions for further refinements to the filter. Finally, some possibilities for additional experiments are discussed. 


\section{Chapter 2}

\section{Sensor Information}

\subsection{Overview of Sensors}

Like any mathematical abstraction of a real-world system, the Kalman filter is a simplified model of reality. For the filter to function optimally, the model must be as complete and as accurate as possible. For this application there are two major aspects of the filter that must be considered. The first concerns prediction: based on knowledge of present system parameters, what will happen to these parameters in the future? The second is more basic: how can knowledge of these system parameters be obtained?

In this chapter, the latter issue is addressed. Each sensor providing input to the filter is described in detail. Using our knowledge of the available sensors, the Kalman filter model can then be developed. Before proceeding further, however, a clarification of the coordinate systems used is required.

\subsection{Coordinate Systems}

Within the filter algorithm, two different coordinate systems are used. The first is earth-referenced, with positions measured relative to fixed reference points on the earth. The second is vehicle-referenced, also referred to as body-referenced. In this case, all measurements are made relative to a specific point on the ROV, usually the center of rotation of the vehicle. Table 2-1 and Figure 2-1 explain the differences in more detail.

An additional coordinate system is sensor-referenced, which depends on the location of the individual sensor. For linear acceleration and velocity measurements, a difference in measurement magnitudes between sensor-referenced and vehicle-referenced measurements exists due to the effects of angular velocities when the sensor is mounted away from the vehicle's center of rotation. The orientation of the axes is the same as vehicle-referenced coordinates since the instruments are mounted colinear with the vehicle axes. Compensation for this difference is discussed in Section 3.5.3. 


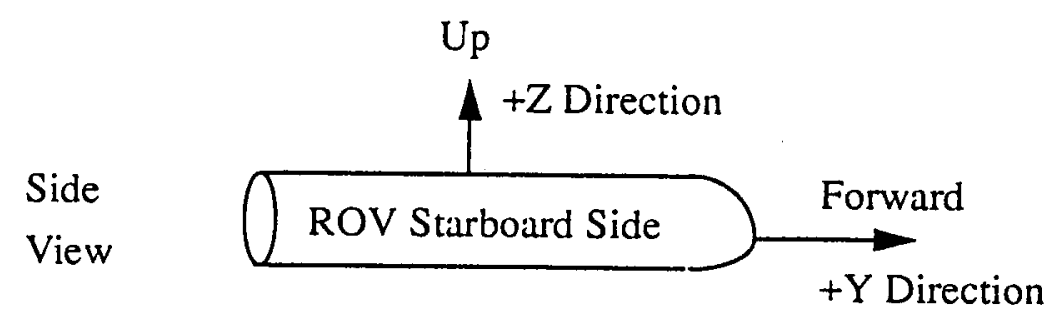

Top

View

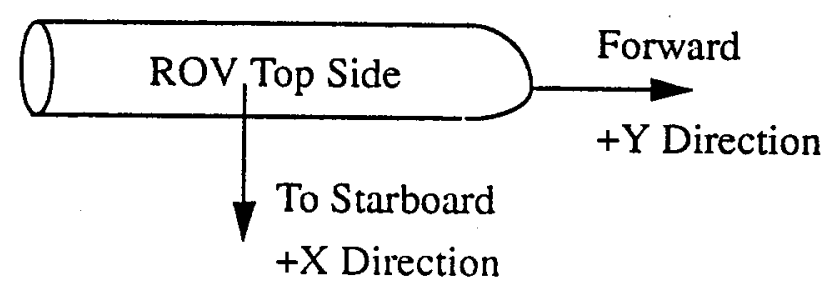

Figure 2-1. Vehicle-referenced coordinate system.

Table 2-1: Comparison of Coordinate Systems

\begin{tabular}{|c||c||c|}
\hline Direction & Earth-Referenced & Vehicle-Referenced \\
\hline $\mathrm{X}$ & Longitude $(+\mathrm{X}=$ east $)$ & $\begin{array}{c}\text { Perpendicular to side of } \\
\text { vehicle }(+\mathrm{X}=\text { to starboard })\end{array}$ \\
\hline $\mathrm{Y}$ & Latitude $(+\mathrm{Y}=$ north $)$ & $\begin{array}{c}\text { In direction of vehicle bow } \\
\text { or stern }(+\mathrm{Y}=\text { forward })\end{array}$ \\
\hline $\mathrm{Z}$ & Depth/Altitude $(+\mathrm{Z}=$ up) & $\begin{array}{c}\text { Perpendicular to top or bot- } \\
\text { tom of vehicle }(+\mathrm{Z}=\text { up })\end{array}$ \\
\hline
\end{tabular}

\subsection{Doppler Velocimeter}

The proper name for this instrument, used to provide vehicle velocities, is the Direct-Reading Broadband Acoustic Doppler Current Profiler (DR-BBADCP). Manufactured by RD Instruments (RDI), the DR-BBADCP is designed to measure current velocities at discrete points through the water column (see Fig. 2-2). Its alternate mode, which is the mode used to gather data for this application, is the bottom-track profiling mode. With the DR-BBADCP mounted on the vehicle looking downward, vehicle 


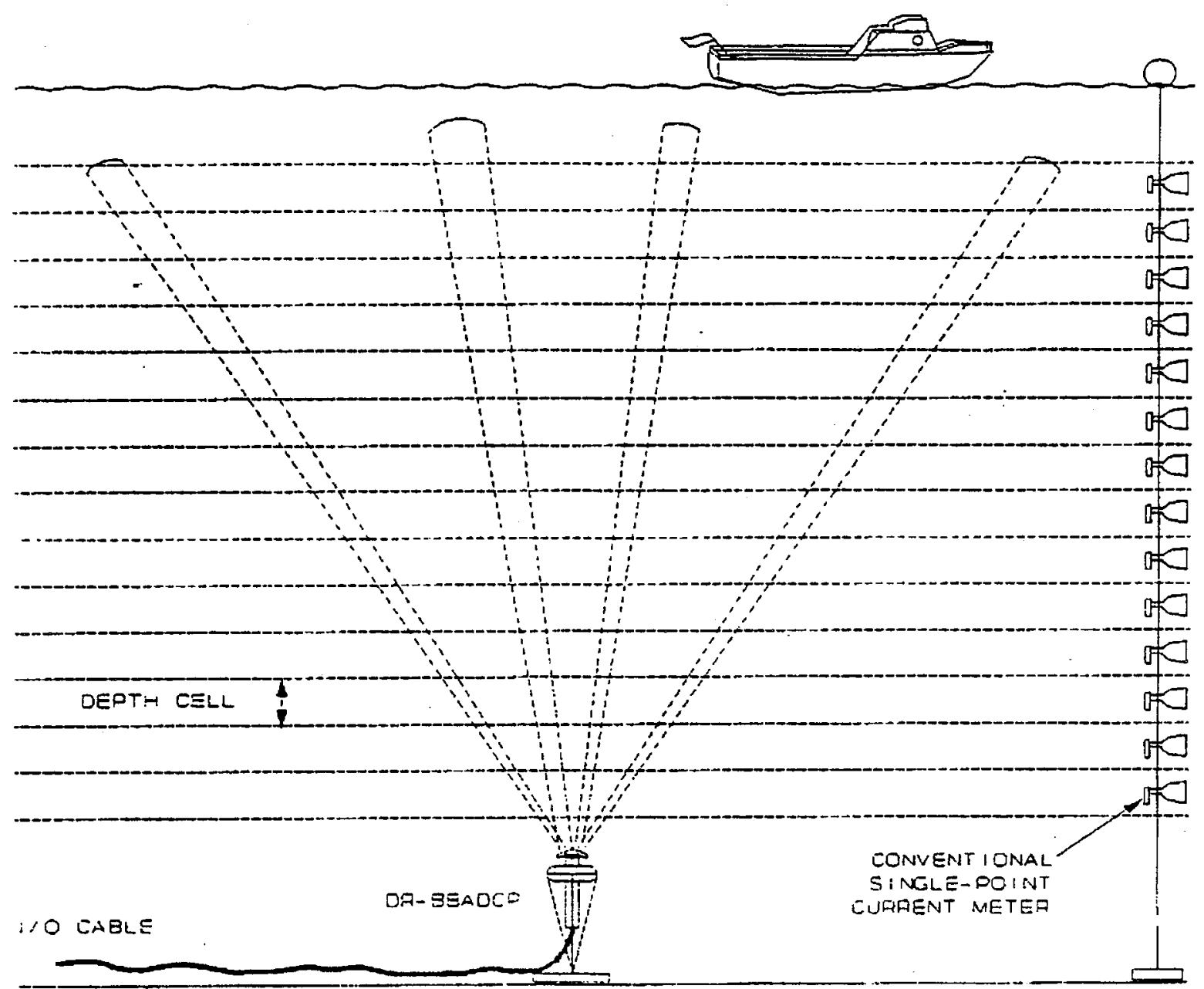

Figure 2-2. Broadband ADCP setup for current profiling (RDI, 1993).

instrument in this application, the more descriptive name of "doppler velocimeter" is used in the remainder of this thesis.

\subsubsection{Principles of Operation}

The doppler velocimeter uses four downward-looking acoustic beams operating at high frequency. Figure 2-3 shows the beam pattern of two of these beams in an upwardlooking mode. RDI manufactures these instruments with six different transmit frequencies: $75,150,300,600,1200$, and $2400 \mathrm{kHz}$. As with any sonar, increasing frequency improves the accuracy but reduces the effective range. Therefore, the frequency 


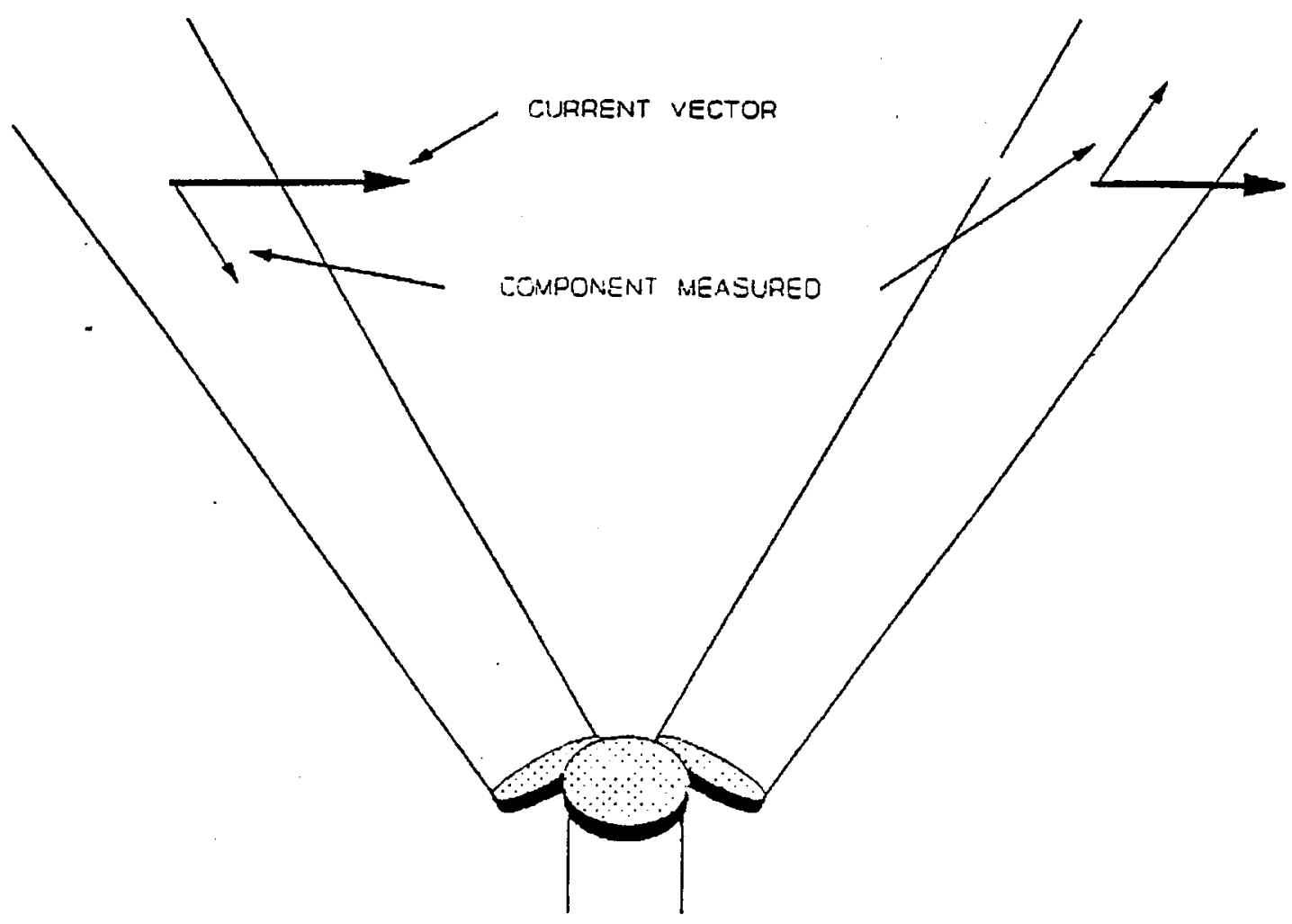

Fig. 2-3. ADCP beam geometry $(R D I, 1993)$.

for a given operation is chosen to be as high as possible while still providing sufficient range to ensonify the bottom based on the expected altitude of the vehicle.

As evidenced by its name, the instrument operates on the doppler principle. Vehicle velocity in the direction of a beam increases the frequency of the returned signal, while velocity away from the beam decreases it. Specifically, the vehicle velocity in the direction of the beam is calculated by $(R D I, 1993)$.

$$
\text { Relative Flow Velocity }(\mathrm{m} / \mathrm{s})=F_{D} \times \frac{c}{2 F_{S}} \text {, }
$$

where

$F_{D}$ is the measured doppler frequency shift in $\mathrm{kHz}$, $c$ is the speed of sound in water at the transducer face in $\mathrm{m} / \mathrm{s}$, $F_{S}$ is the transmitted acoustic frequency in $\mathrm{kHz}$. 


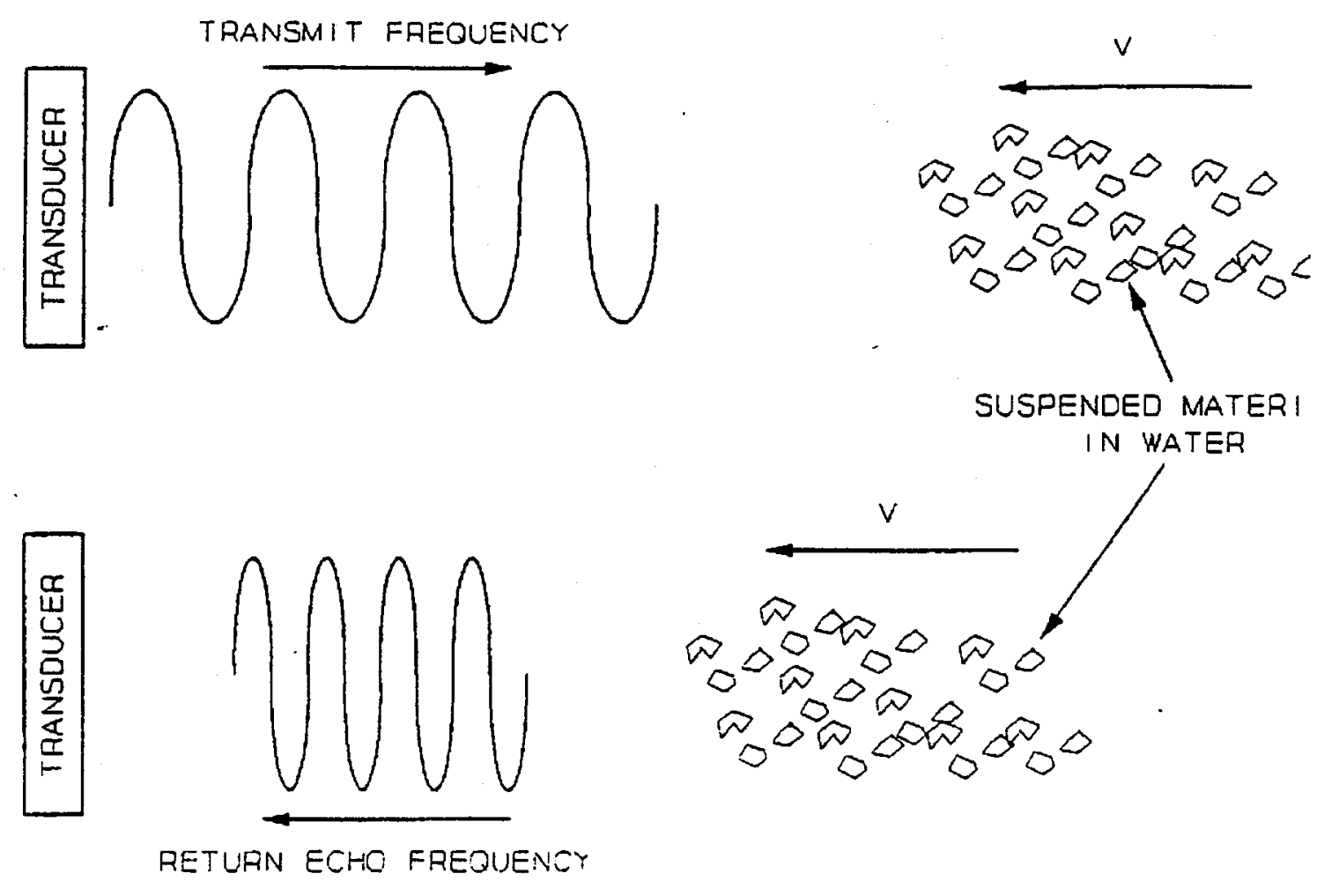

Fig. 2-4. Frequency change caused by doppler effect $(R D I, 1993)$.

Figure 2-4 shows this effect with a fixed transducer and moving material in the water. The effect is the sume with the transducer mounted on a moving vehicle over the fixed bottom. The four beams transmit at different angles so the velocities in each direction can be resolved. Three are needed to solve for velocities in all three directions and the fourth provides redundancy to ensure accuracy.

To interpret the received sonar data, the doppler velocimeter uses an autocorrelation processor. A series of short pulses is transmitted, each with a pulse length of $T_{P}$ with a known lag $T_{L}$ between pulses. When the returns are received, the processor compares the phase change between two distinct pulses, accounting for the difference in time between their respective transmissions. As shown in Fig. 2-5, a zero phase change implies zero velocity. Likewise, a phase change equates to a velocity with magnitude determined by the amount of phase change. Phase changes with a magnitude greater than $2 \pi$ are resolved using a proprietary RDI algorithm using different subsets of the - 


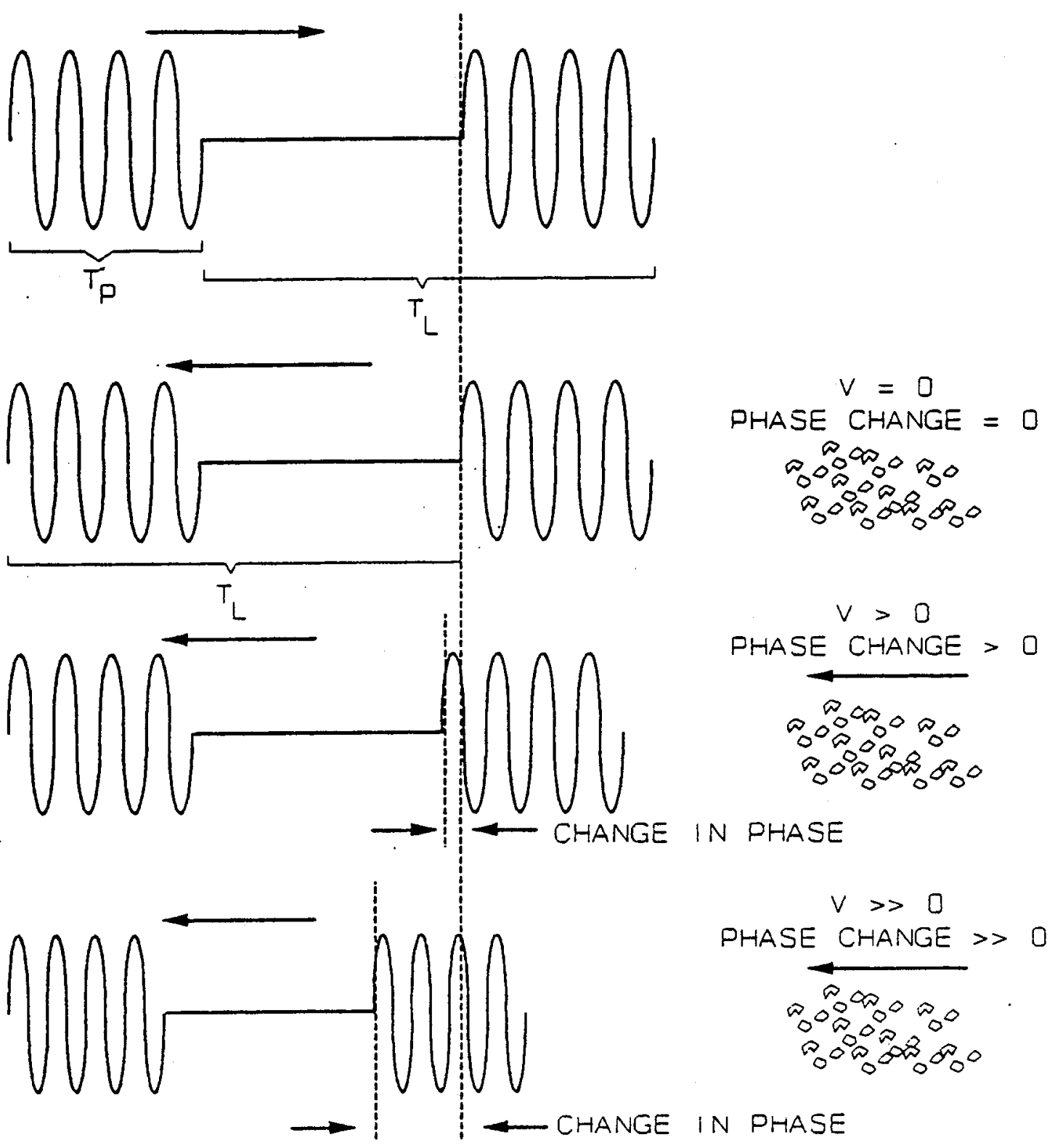

Fig. 2-5. Phase change seen by the broadband system $(R D I, 1993)$.

transmitted pulses. If any datum is below the operator-selector minimum for correlation magnitude, that datum is rejected by the doppler velocimeter's processor.

The output of the velocimeter can be referenced four different ways. The most basic provides measurements of the velocities relative to each of the four beams. Alternatively, the velocimeter's internal processor can resolve the components of the four beams to provide velocity fore or aft, velocity left or right, and velocity up or down 
relative to the instrument. Third, if the instrument is not mounted on a ship or ROV with the same orientation as the platform's reference axes, the velocities can be converted to any desired direction relative to the platform using knowledge of the difference between the instrument axes and platform axes. Finally, the doppler velocimeter can use its internal flux-gate sensor, which uses heading and pendulum sensors to provide pitch and roll. The internal processor can use this roll, pitch, and heading information or similar data from external sensors to convert from vehicle-referenced to earth-referenced velocities.

For this Kalman filter the velocimeter is mounted with axes oriented the same as the vehicle axes described in Section 2.2. Therefore, a coordinate transformation to the platform axes is unnecessary except for any correction due to angular velocities. Instead of allowing the velocimeter's internal processor to convert velocities to earth-referenced coordinates, which would use the current output of the appropriate sensor for attitude measurements, the filter uses the vehicle-referenced values. Using vehicle-referenced velocities allows the filtered estimates of roll, pitch, and heading to be used to make the conversion to earth-referenced coordinates. This improves overall filter performance since the filtered estimates are less noisy than the instantaneous sensor outputs.

\subsubsection{Inputs to Velocimeter}

To provide the desired output, the doppler velocimeter uses the speed of sound in its calculations of vehicle velocity. As is the case for data used in this thesis, this speed of sound is typically entered manually by the operator to permit easier post-processing of the data. However, for more accurate calculations the doppler velocimeter can calculate the current speed of sound using three inputs: sulinity, depth, and temperature.

For most open-ocean operations salinity is constant. Therefore, salinity can be manually inserted prior to operations. If operations are to be conducted in an area of varying salinity, such as near a river mouth, then an external conductivity sensor can be used to provide salinity information to the velocimeter.

Since the majority of vehicle operations occur within a relatively narrow depth band, making speed of sound changes due to depth change negligible, depth is also normally manually inserted. If operations involving large depth changes are expected, 
consideration should be given to providing depth estimates to the velocimeter to calculate a variable speed of sound.

Finally, the doppler velocimeter has an internal temperature sensor. Manual input can be used in this case as well. For operations near the bottom in deep water, where thermal gradients are negligible, manually inputting temperature permits more precise post-processing of the data without introducing significant errors. In areas with larger gradients, using the installed temperature sensor is necessary.

\subsubsection{Doppler Velocimeter Outputs}

Obviously, the primary output desired from the doppler velocimeter is vehiclereferenced velocity. However, there are several operator-selected options that affect the accuracy and the frequency of these measurements.

Frequency of output is determined by three factors: the speed of sound in the water, the range to the bottom (vehicle altitude), and the number of pings per ensemble. The doppler velocimeter averages the results from the pings in each ensemble to improve accuracy. The technical manual recommends four pings per ensemble. A larger number of pings per ensemble results in better quality measurements but at the cost of a lower update rate. Using the recommended four pings per ensemble, an average altitude of $100 \mathrm{~m}$, and the nominal speed of sound in seawater of $1500 \mathrm{~m} / \mathrm{s}$, the time between velocity measurements can be calculated as

4 pings $\times 200 \mathrm{~m} /$ ping $\times 1 \mathrm{~s} / 1500 \mathrm{~m}=0.53 \mathrm{~s} / \mathrm{ensemble}$.

The standard deviation for velocity measurements is computed by the velocimeter's processor based on the BBADCP frequency (for the model in use), the range to the bottom, the number of pings per ensemble, and the size of the depth cells selected prior to operation. The size of the depth cell is important for precision when operating in the water column profiling mode. For bottom tracking, however, the largest depth cell should always be used since this gives the lowest standard deviation for vehicle velocity measurements. For vehicle operations treated in this thesis, the standard deviation is on the order of 1-2 cm/s. Figure 2-6 shows how accuracy varies with range for a single ping using the $150-\mathrm{kHz}$ model. This high accuracy enables precise measurement of 


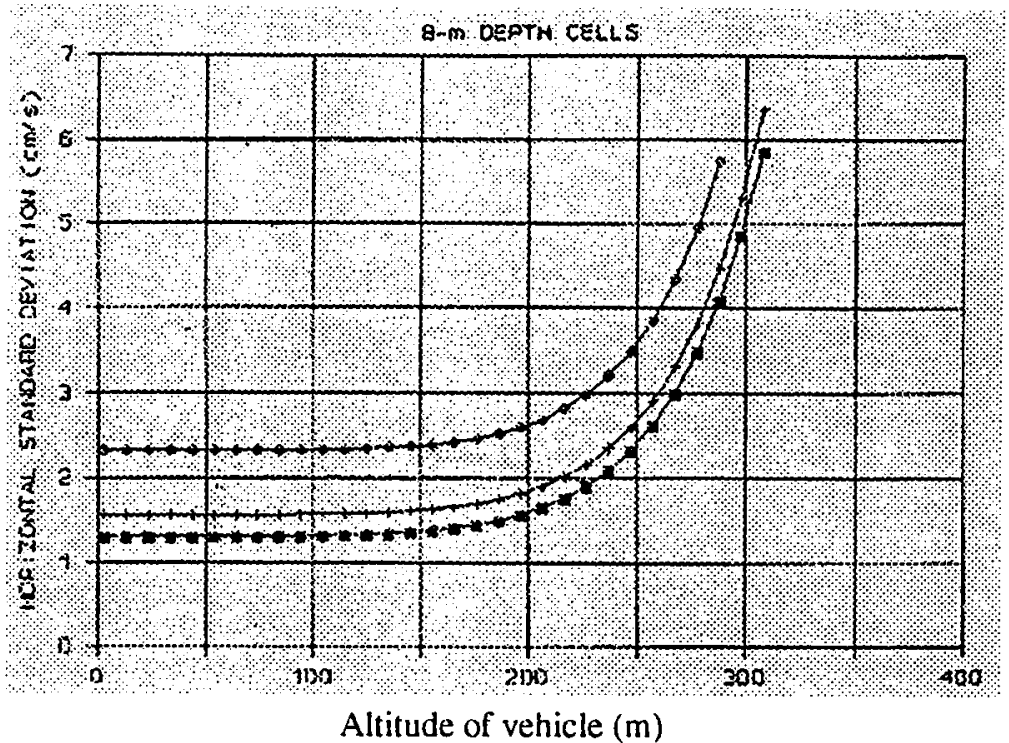

Fig. 2-6. Standard deviation for a single ping using the $150-\mathrm{kHz}$ system (with ADCP velocity, from top to bottom, of 20 knots, 10 knots, and 0 knots) $(R D I, 1993)$.

velocity magnitude. However, overall system accuracy is limited by how well heading, pitch, and roll can be estimated. Since standard deviation is constantly updated by the doppler velocimeter's software, that information can be included in the Kalman filter algorithm. Specifics of this implementation are discussed in Chapter 3.

\subsection{Depth Sensor}

The depth sensor used for this work is manufactured by Paroscientific, Inc. and consists of a quartz-crystal resonator whose frequency of oscillation varies with pressureinduced stress. The sensor also includes thermal compensation using a quartz-crystal temperature signal. Nominal accuracy of the sensor is $0.02 \%$, and a reading can be obtained approximately every 0.25 s. (Paroscientific, 1987).

For the purposes of achieving the best overall estimates of vehicle position and velocity, the absolute error of this sensor is less important than its stability. Previous experience indicates that the depth sensor should be very consistent in its outputs. Therefore, the output of the sensor is a vital element in increasing the accuracy of the 
Kalman filter used to determine state space estimates for depth-dependent vehicle parameters.

The only time the sensor should exhibit less stability than desired is if the sensor is not in thermal equilibrium with the surrounding water. This could occur soon after initial deployment. However, in this case the problem would be foreseen, and thermal equilibrium could be achieved prior to commencing actual search operations. Of more concern is operation in water with strong thermal gradients. This problem could be particularly acute in shallow-water operations, especially at certain times of the day. During such operations, the pressure sensor can be expected to give less accurate results, resulting in greater Kalman filter errors.

\subsection{External Positioning System}

- There are several possible systems that can be used to provide external position information. Historically, nearly all underwater vehicle positioning information has been obtained from some type of acoustic net. These acoustic systems generally fall into three different categories, with the type used for a specific operation dependent on the type of mission and the topography of the bottom.

\subsubsection{Long-Baseline Systems}

In a long-baseline system, an array of acoustic transponders is deployed on the bottom in the vicinity of projected vehicle operations. A transponder is also attached to the vehicle. Vehicle position is determined by measuring the travel times of sound waves between the vehicle and the transponders in the net. For two-way systems, the vehicle sends out a ping, and each transponder responds with a coded return signal when it receives the initial ping from the vehicle. The travel times are then translated into range. One-way systems use the same principle, but travel time is measured only from the vehicle to each transponder in the net. With either system, since the positions of the transponders on the bottom have already been determined when calibrating the acoustic net, the position of the vehicle can be determined (Morgan, 1978). 
An example of such a system is the Sonic High Accuracy Ranging and Positioning System (SHARPS). This commercial system uses three transceivers near the ocean bottom, which are connected to a surface ship by coaxial cables. Another transceiver is mounted on the ROV, which is also connected to the ship. Because the cable connections allow the surface ship to know the exact transmission time of each ping, only one-way travel timies are needed, which allows twice the frequency of updates as a two-way system. Operating at $300 \mathrm{kHz}$, SHARPS is limited to a range of $100 \mathrm{~m}$ in seawater but can provide accuracies on the order of $2 \mathrm{~cm}$ (Somers, 1991).

A disadvantage of SHARPS is the need for multiple cables from the surface ship to the transponder field, which can cause difficulties when maneuvering a ROV in the area. Another system, EXACT, was used by Yoerger and Mindell to control the ROV Jason in the vicinity of an active hydrothermal plume at a depth of 2200 meters. The EXACT system operates similarly to SHARPS and provides comparable accuracies but uses no cable connections to the surface. Instead, the master unit on the ROV communicates with the surface through a low-bandwidth serial link. Because there are no cable connections, two-way travel times are used (Yoerger and Mindell, 1992).

The major advantages of a long-baseline system are its stability and its accuracy when conducting vehicle operations in deep water close to the bottom. Because the transponder array is on the seafloor; it is fixed once placed and calibrated. Therefore, its accuracy is improved over a less stable system. Since the array of transponders is deployed on the bottom near the area of operations, the difference in acoustic travel times between each pinger and the vehicle will be greater than if all pingers were located close to the surface, which also enhances accuracy. Finally, the acoustic net can be made as large as desired. By using lower frequency systems, the maximum range to the ROV can be greatly increased at the cost of reduced accuracy and decreased update frequency.

There are two major disadvantages of this method. First, because a transponder net must be deployed on the bottom and calibrated, significant preparation time is required before ROV operations can begin. Second, vehicle operations are restricted to the vicinity of the acoustic transponders. Therefore, for large-area surveys, this method is impractical. In this case, a short-baseline system may be used (Morgan, 1978). 


\subsubsection{Short-Baseline Systems}

The principles of operation of the short-baseline systems are similar to those having a long baseline. In fact, a system such as SHARPS can be used in either configuration. The obvious difference is that instead of the acoustic net transponders being placed on the ocean floor, they are attached to the surface ship. This method eliminates some disadvantages of the long-baseline system. Since the transponders are mounted on the surface vessel in known locations, no initial surveying is required. Also, the ship can be moved wherever operations are desired, so the range limitations of a bottom-fixed net are negated.

Like the long-baseline system, the short-baseline system measures the difference in arrival time from the acoustic pinger on the ROV to each transponder on the ship. However, because the geometry of the ship-mounted array is precisely known, this difference in arrival times can be converted into an angular direction from the plane of the array to the ROV. Since the ROV is tethered to the ship, the time of each ping is also known precisely. Therefore, both beuring and range information are available, yielding the location of the ROV (Morgan, 1978).

However, the short-baseline system tends to increase position errors because of the more sensitive geometry. Also, because the transponder array is now mounted on a moving platform, surface positioning errors can become the dominant factor in determining vehicle location. The importance of accurately measuring the surface ship's heading, pitch, and roll also become vital, especially when the range to the ROV increases, since errors in these measurements result in an erroneous measurement of bearing to the ROV (Somers, 1991).

\subsubsection{Ultrashort-Baseline Systems}

The ultrashort-baseline system usess a hydrophone array mounted either on the ROV or the surface ship, with only a single pinger on the other platform. Normally, the hydrophone array is on the surface ship. Phase comparisons rather than travel-time measurements are used to determine the position of the vehicle. The acoustic pinger sends out a pulse, and the processor connected to the hydrophone array measures the phase 
difference in the signals received at each hydrophone. This phase difference is converted into a bearing to the pinger. For tethered vehicles, the time of travel can also be computed as in the short-baseline case to obtain range. Therefore, ROV position can be determined. To measure phase difference accurately, the receiving hydrophones are mounted within one wavelength of each other (typically, only centimeters apart), making the array small enough för use on ROVs.

This system has two major disadvantages. First, the installation must be extremely precise, which can be difficult. Second, multipath arrivals of the sound waves via bottombounce or surface-reflection can cause false phase measurements resulting in large inaccuracies. In his 1992 thesis, Brian Tracey explores techniques to minimize the effects of this multipath interference (Tracey, 1992).

\subsubsection{Other Positioning Systems}

Besides traditional acoustic positioning systems, other possibilities for determining vehicle position exist. For example, research is being performed at DSL and other laboratories in making terrain-relative mapping feasible. This method entails matching bottom features seen by the vehicle with known features from an existing database, or with features already found and mapped by the vehicle. Depending on the features present in the vicinity, the quality of the database, and such system characteristics as sonar frequency, position fixes with accuracies on the order of meters are foreseeable.

\subsubsection{Position Information Required for Use in the Kalman Filter}

For the purposes of constructing the filter, any of these system configurations can be used, but allowances must be made to account for the differences between them. A longer range system, using lower frequencies, is typically less accurate. Additionally, since the frequency of fixes is limited by the speed of sound in the water, the longer range system will obtain fixes less frequently. The filter must be adjusted accordingly. 


\subsection{Gyro}

The gyro used at DSL is manufactured by Humphrey, Inc. It is a standard uncompensated model that provides continuous heading information. It maintains excellent short-term precision with little "jitter," or high-frequency fluctuation. However, because it is uncompensated, it has a high drift rate that tends to fluctuate from three to six degrees per hour. This drift introduces a significant and variable bias that can have a debilitating effect on the Kalman filter, which assumes only zero-mean Gaussian errors. To correct for this bias, it is included in the filter state space so that it can be continuously adjusted as necessary based on other heading measurements.

\subsection{Flux-Gate Magnetic Compass}

The magnetic compass used at the DSL is the C-100, manufactured by KVH Industries, Inc. It consists of a toroidal flux-gate sensing element with an associated electronics board. The sensor element is a saturable ring core, which floats in an inert fluid so the sensing element remains horizontal. Windings surround the lexan housing of the sensor element, electrically driving the coil into saturation. Secondary windings then sense pulses generated by the horizontal component of the earth's magnetic field ( $K V H$, 1992).

The compass provides heading information at $10 \mathrm{~Hz}$. The manufacturer's specifications list the accuracy as $0.5^{\circ}$, with a $0.1^{\circ}$ resolution. However, there are three major obstacles to achieving this accuracy when the compass is mounted on a vehicle.

First, the magnetic compass is referenced to magnetic north rather than true north. Therefore, the difference between true and magnetic north, or variation, must be compensated for prior to using the heading information for the filter. This variation is dependent on the location of the vehicle. While the average variation in an area can easily be obtained from the pertinent navigational chart, the actual compass reading can be affected by local concentrations of iron beneath the bottom of the sea. Since the vehicle is usually operating within $200 \mathrm{~m}$ of the ocean bottom, where local magnetic disturbances 
can be present, performance of the magnetic compass must be monitored to ensure that any magnetic disturbances are recognized.

The second error is deviation, or the difference between a perfect magnetic compass and the compass in use. This is influenced by the construction of the compass as well as the presence of ferrous metals on the vehicle in the vicinity of the compass.

The third source of error is more problematic. Whenever the vehicle's thrusters are used, the resulting magnetic field from the energized motors affects the output of the compass, resulting in output instability until the thruster-induced magnetic transients decay. For large-area mapping operations, the vehicle operates mostly on long, straight legs, so the effects of thruster-induced errors are minimized. Operations requiring more frequent maneuvering degrade compass accuracy accordingly.

The characteristics of the magnetic compass are the opposite of the gyro: it does not drift with time but does exhibit significant short-term fluctuations. Because of its lack of drift, it can be used as a reference to reset the gyro when required. However, care must be taken to ensure that the magnetic compass is settled out when performing the reset. Most importantly, the vehicle thrusters should not be in operation during this procedure to avoid the subsequent error in compass heading output. As for the gyro, magnetic compass bias is included in the state space to provide a continuously updated estimate of its value.

\subsection{Inclinometer}

At DSL, the single-axis Watson inclinometer with angular rate sensor is used to provide pitch and roll information. This unit combines accuracy and reliability with small size and low power requirements, making it ideal for vehicle operations.

The inclinometer uses a rate sensor and an integral vertical reference. A position output is obtained by integrating the angular rate output. By comparing this signal with the vertical reference, an error signal is generated. This error signal is filtered and sent back to the rate sensor as a bias. The system is also damped. With the overall system, the effects of inertia, damping, and short-term accelerations are reduced.

The angular rate sensor uses two piezoelectric bender elements, which are resonantly driven in opposite directions. Rotation causes a bending force, which is 


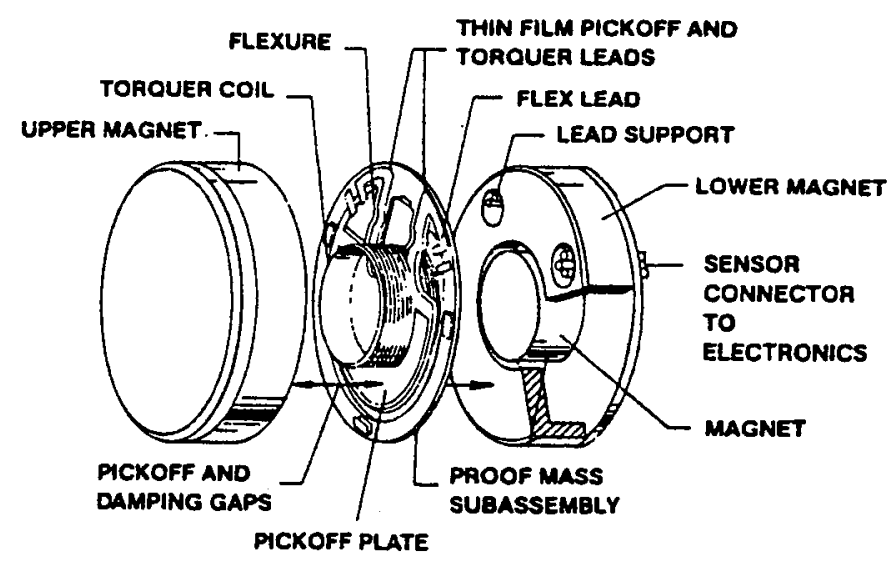

Fig. 2-7. Accelerometer sensor exploded view (Cady, 1984).

demodulated to produce the rotation rate. The vertical reference uses a liquid-capacitive element. With these components, the system has no moving parts, which increases reliability. Overall, the system weighs less than eight ounces, making it easy to mount on a ROV (Watson, 1990). To obtain the necessary data, two of these units are used on the vehicle, mounted orthogonally. One provides pitch, the other roll.

\subsection{Systron Donner MotionPak (Inertial Motion Unit)}

The Systron Donner MotionPak provides the final sensor measurements required for the Kalman filter. It consists of two parts: the linear accelerometers and the angular velocimeters.

\subsubsection{Accelerometer Operation}

The linear accelerometer used for vehicle navigation in this application is the Sundstrand Data Control Q-Flex® accelerometer (see Fig. 2-7). Acceleration produces a torque on the sensor's proof mass. A detector measures the displacement of the mass and produces a proportional output voltage. The resultant signal is amplified and fed to a torquer coil fixed to the proof mass. This current in the coil provides a restoring torque to balance the applied acceleration. The current also goes through a load resistor, generating 


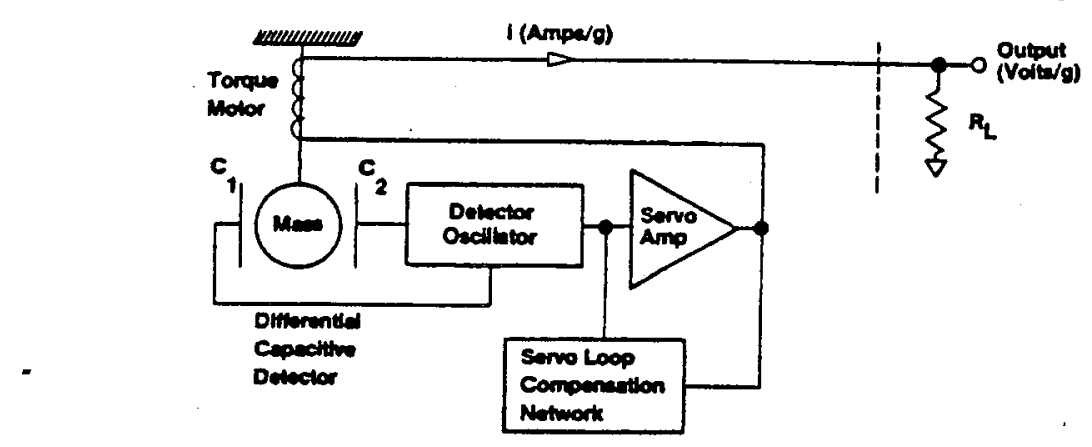

Fig. 2-8. Accelerometer system functional diagram (Cady, 1984).

the output voltage for the detector (Cady, 1984). See Fig. 2-8 for an overall system schematic.

\subsubsection{Angular Velocity Measurements}

Angular measurements are provided within the IMU using the Systron Donner GyroChip $^{\mathrm{TM}}$. This device uses a vibrating quartz tuning fork to sense angular rate by acting as a Coriolis sensor. The vibrating fork drives a similar pickup fork that produces the output signal. These two forks, along with their support flexures and frame, are made from a wafer of single-crystal piezoelectric quartz. The drive tines are driven by an oscillator that causes the tines to move toward and away from each other at high frequency. Each tine has a coriolis force acting on it given by

$$
F=2 m \omega \times V_{r},
$$

where

$\mathrm{m}$ is the tine mass,

$\omega$ is the input rate,

$V_{r}$ is the instantaneous linear radial velocity.

The forces generated are perpendicular to the plane of the fork assembly at each of the tines and in opposite directions, yielding a torque proportional to the input angular velocity. The pickup tines respond to the oscillating torque by moving in and out of the plane, causing output signals to be produced by the pickup amplifier (Systron Donner, 
1993). A separate unit is provided for each axis, resulting in independent measurements for pitch rate, roll rate, and yaw rate. 


\section{Chapter 3}

\section{Development Of The Kalman Filter . Model}

\subsection{Characteristics of the Linear Kalman Filter}

The purpose of the Kalman filter is to produce an unbiased, minimum variance, consistent estimate of a quantity $\underline{x}$ based on a set of measurements, $\underline{z}$, where the relationship between these variables can be represented by

$$
\underline{z}=H \underline{x}+\underline{y} .
$$

Therefore, $z$ is a linear combination of the elements of the vector $x$, plus random noise represented as $\underline{\underline{y}}(\mathrm{Gelb}, 1974)$. The desired properties of such an estimate, as delineated above, are:

Unbiased: the expected value of the estimate is the same as that of the actual quantity being estimated.

Minimum Variance: the error distribution of the estimate is less than or equal to that of any other unbiased estimator.

Consistent: as the number of measurements increases, the estimate converges to the true value of the quantity being estimated.

If the noise is Gaussian and the relationship is linear, the Kalman filter has been proved to meet these criteria (Gelb, 1974).

An additional characteristic of the Kalman Filter is that it is recursive, which means that new estimates can be computed without storing past measurements. Instead, all previous information is summarized in the current estimate and associated error covariance matrix. This recursiveness allows real-time processing of data without excessive memory storage requirements or computational burden. 


\subsubsection{System Model}

For a given real system to be represented using a Kalman filter, it must be put into a standard form. The two governing equations of the system model are:

$$
\underline{\dot{x}}=A \underline{x}+G \underline{w}+L \underline{\underline{u}}
$$

and

$$
\underline{z}=C \underline{x}+D \underline{u}+\underline{v}
$$

where $\underline{u}$ is a deterministic control input, $\underline{w}$ is a random forcing function with error covariance $Q$, and $\underline{y}$ is the measurement noise with error covariance matrix $R . A, G, L, C$, and $D$ are matrices describing the relationship between the different vectors.

The first equation states that the rate of change of the system parameters can be predicted using a linear combination of the present parameters and a linear combination of control inputs. The second states that the measurement vector, $z$, is a linear combination of the system parameters plus a linear combination of the present control inputs. Both equations include the effects of noise by virtue of the $\underline{w}$ and $\underline{w}$ terms.

\subsubsection{System Model Dynamics}

To produce an appropriate Kalman filter model, the state variables that make up the vectors $x$ and $z$ must be defined. The vector $x$ includes all parameters necessary to model vehicle behavior. The vectorzi includes all measurements that can be obtained from the sensors as described in Chapter 2. Both must be determined using knowledge of the system dynamics and the available sensors. In this case, there are two different types of model propagation, one for vehicle motion and one for attitude.

The primary control forces are those induced on the vehicle by the tow cable. However, these àre virtually impossible to predict due to the realities of the towing operation. First, although the surface ship is proceeding predominantly on a known course, wave action produces continuous variations in pitch, roll, and heading. These variations are all propagated down the tow line to some degree, despite various techniques used to isolate vehicle motion from ship motion. Even if these forces on the tow cable at 


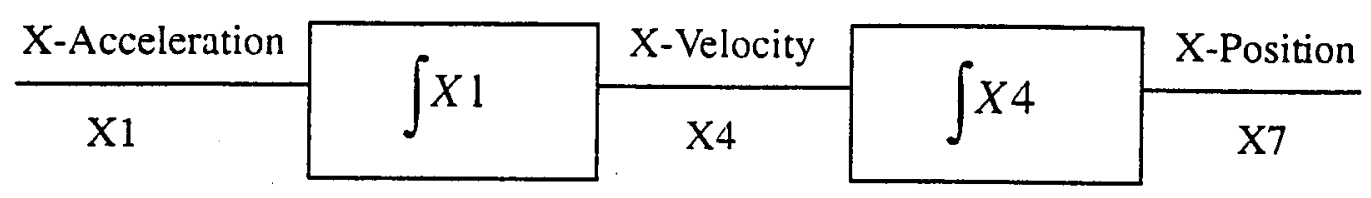

Fig. 3-1. System model for X-position propagation.

the surface could be measured precisely, the cable forces at the vehicle, several thousand meters away, are difficult to model.

The vehicle model is also ill-defined. Changes in equipment configuration on the vehicle, which affect drag and added mass, can have significant effects on the vehicle dynamics. Also, when cable forces induce a change in vehicle attitude, the force of the water acting on the moving vehicle coupled with the designed self-righting characteristics of the vehicle combine to restore the velicle to its mean velocity and attitude. Thus, the vehicle frequently follows a damped sinusoidal path in both vertical and horizontal directions. Therefore, the predominant characteristics of vehicle motion are:

1. It is extremely unpredictable due to the inability to measure cable forces and to properly model vehicle dynamics.

2. The vehicle tends to return to its mean attitude after a cable disturbance has subsided.

As a result, the vehicle accelerations are be considered to be influenced by a whitenoise disturbance and control inputs are not modelled. To help compensate for any errors in these assumptions, linear accelerations are measured by the strapdown inertial motion unit (IMU) to provide current, though noisy, actual accelerations to the filter algorithm.

For thruster-controlled vehicles, control input is often better defined. In these circumstances, operator-controlled inputs can be added as a deterministic vector $\underline{u}$, with an associated matrix $L$ to mathematically relate the inputs to their effect on the state vector.

To estimate vehicle position, the system model is as shown in Figure 3-1. Xposition (latitude) is shown; Y-position (longitude) and Z-position (depth) follow the same principles. 


\begin{tabular}{l|l} 
Roll Rate & Roll \\
\hline X10 & X10
\end{tabular}

Fig. 3-2. System model for vehicle propagation.

\begin{abstract}
The system model for the angular positions is similar but requires only one integrator. Again, the system is modelled as disturbed by white noise. The angular velocity output of the IMU is used as the measurement for angular velocities (roll, pitch, and heading), shown in Figure 3-2.
\end{abstract}

\title{
3.2 Sensor Outputs and Their Relationship to the State Space
}

Before proceeding to the specifics of the state space model for this application, some details of the sensor data output must be described. To produce a useful Kalman filter for this application, it is essential to identify exactly what information is available. Additionally, an expected frequency for receipt of data by the filter is necessary.

During vehicle operations conducted by DSL, four different data packages are collected as the appropriate data are available. These four data streams must be translated into a format usable by the Kalman filter, and any potential gaps in receiving data must be anticipated and accounted for in the filter algorithm.

\subsubsection{IMU Package}

The IMU package consists of the linear accelerometers and angular velocimeters. As is the case for the other three data packages, the raw data stream is translated into a usable form for the filter using a relatively simple program written in $C$ language. The IMU package provides data at a rate of approximately $10 \mathrm{~Hz}$. The IMU's outputs are all in sensor coordinates, which must be converted to body coordinates for use in the filter. 


\subsubsection{Attitude Package}

The attitude package combines the outputs of the depth detector, inclinometers, gyro, and compass to provide depth, roll, pitch, and heading. While individual sensors providing data operate at various frequencies, the overall package operates at approximately $5 \mathrm{~Hz}$.

\subsubsection{Doppler Velocimeter Package}

Included in each data stream from the doppler velocimeter is considerable detail about the operator-selected parameters being used by the velocimeter. However, only the body-referenced velocities in each orthogonal direction and the velocity error measurement are used by the Kalman filter. Again, a $C$ program is used to translate the Hex-ASCII output into usable data, and the sensor-referenced outputs must be converted to body coordinates.

As discussed in Chapter 2, the output frequency of the doppler velocimeter depends primarily on the number of pings per ensemble and the altitude of the vehicle above the bottom. For the sidescan-sonar mapping configuration employed during the DSL-120 deployment used for this thesis, the doppler velocimeter usually provided data approximately every 2-3 s. However, this time period was occasionally shortened or lengthened due to changing altitude and varying bottom conditions.

\subsubsection{Positioning System Package}

The positioning system has the most irregular of the four data streams received for vehicle navigation. Frequency of data reception depends on the type of positioning system in use. For systems using an acoustic transponder array, such as the long-baseline or shortbaseline systems, this depends primarily on the distance from the vehicle to the acoustic transponder array. However, whenever data acquisition is secured (usually when a given track line is completed until the towing ship has maneuvered to get into position for the next track line), the positioning system is frequently secured as well, since precise knowledge of vehicle location is unnecessary except when obtaining sonar data. Of more concern is a loss of position information during sonar operations. There are several 
different possibilities that can cause such a problem. A large bottom feature, such as a ridge, can physically block the path from the acoustic array to the vehicle. Another significant factor is the sound-velocity profile (SVP), which dictates the path sound waves will travel in the water. With an unfavorable SVP, acoustic position information can be lost only a short distance away from the transponder array.

For systems not tied to an array, such as a terrain-relative positioning system, the frequency of obtaining information will be highly unpredictable since it depends on the available terrain and the quality of the database. It is likely that there will be frequent long gaps between fixes and that these gaps will exist whether or not sonar operations are in progress.

This irregularity of position information is an important reason for developing a Kalman filter using the doppler velocimeter information. By filling in the gaps in position fixes with an accurate estimate of vehicle position, the sonar data quality can be greatly enhanced.

Like the attitude data stream, the position data stream is typically easy to decipher. The components used for this Kalman filter are the $X$ and $Y$ positions (in meters from a reference point) and the time the datum was obtained.

\subsection{Defining the State Space}

Now that the system dynamics and available measurements are clearly identified, the state space can be defined. Once the.r vector is labeled, the Kalman filter model can be mathematically developed. Using the coordinate system axes specified in Section 2.2, the components of the state vector.

$X 1: X$-acceleration (All accelerations are in body, or vehicle-referenced, coordinates)

X2: Y-acceleration

X3: Z-acceleration

$\mathrm{X} 4$ : $\mathrm{X}$-velocity (All velocities are in body, or vehicle-referenced, coordinates)

X5: Y-velocity

X6: Z-velocity 
$\mathrm{X} 7: \mathrm{X}$-position (All positions are in earth-referenced coordinates)

X8: Y-position

X9: Z-position

X10: Roll rate (Defined as rotation about the vehicle's Y-axis; positive roll is defined as port side up)

X11: Pitch rate (Defined as rotation about the vehicle's X-axis; positive pitch is defined as bow up)

$\mathrm{X} 12$ : Yaw rate (Defined as rotation about the vehicle's $Z$-axis; heading is measured with normal compass orientation. To maintain the right-handedness of the system, this is converted within the Kalman filter algorithm to measure positive heading rate, usually called yaw rate, as counterclockwise, rather than the normal compass direction of clockwise)

X13: Roll

X14: Pitch

X15: Heading

X16: Gyro bias (Difference between gyro heading and true heading)

X17: Magnetic compass bias (Difference between compass heading and true heading. It includes both variation and deviation)

Also, there is the measurement vector, $z$. When all four data streams are available to the filter, the components of $z$ correspond exactly to the components of the state vector $x$, with the addition of two heading measurements from the gyro and compass. As discussed later, however, this is seldom the case due to the variations in data stream frequency.

\subsection{Nonlinearity and the Extended Kalman Filter}

Unfortunately, the differences in coordinate systems between the various sensors means that the model cannot be treated as linear. With vehicle accelerations and velocities provided in body coordinates and positions provided in earth-referenced coordinates, a coordinate transformation must be made between the two references to allow the data to be used. This coordinate transformation is accomplished using standard trigonometric equations that use vehicle roll, pitch, and heading to relate body coordinates to earth 
coordinates. This transformation is performed within the extended Kalman filter algorithm by altering the $A$ matrix at each filter iteration as explained in Section 3.7.2.2

\subsubsection{Choosing the Extended Kalman Filter}

As stated in Section 3.1, the linear Kalman filter has been proved to provide an unbiased, minimum-variance, consistent estimate of the state vector $x$ as long as the noise is Gaussian. Noise considerations are addressed in Section 3.5.

To compensate for the nonlinearity of the system, several techniques are available. Unfortunately, none of these can be proved to meet the same standards as the linear Kalman filter. In order of complexity, three possible solutions are the extended Kalman filter, the iterated extended Kalman filter, and the second-order filter (Gelb, 1974).

Because it is the simplest and incurs the least computational burden, the extended Kalman filter is used to provide the state estimates for this application. As Gelb states,

There is no guarantee that the actual estimate obtained will be close to the truly optimal estimate. Fortunately, the extended Kalman filter has been found to yield accurate estimates in a number of important practical applications $(\mathrm{Gelb}, 1974)$.

\subsubsection{Filter Propagation}

The basic method of producing a state estimate with the extended Kalman filter is the same as for the linear version. First, some definitions must be provided:

$\underline{x}(t \mid t)$ is the estimate of the state vector using all data up to and including the present time.

$\underline{x}(t+1 \mid t)$ is the predicted estimate of the state vector at the time of the next filter update step using all data up to and including the present time.

$\underline{x}(t \mid t-1)$ is the predicted estimate of the state vector at the present time using data up to and including the time of the last filter step. It is the same as the. $x(t+1 \mid t)$ calculated after the previous filter update step.

$P(t \mid t)$ is the error covariance matrix of the state vector using all data up to and including the present time.

$P(t+1 \mid t)$ is the predicted covariance matrix of the state vector at the time of the next filter update step.

$P(t \mid t-1)$ is the predicted estimate of the error covariance matrix at the present time 
using data up to and including the time of the last filter step. It is the same as the $P(t+1 \mid t)$ calculated after the previous filter update step.

For the linear Kalman filter, the error covariance matrices are deterministic and exact based on the assumptions used for input and measurement noise in the filter model. Therefore, they provide the current uncertainty of each variable in the state vector, assuming the model is correct. For the extended Kalman filter, the error covariance is only an approximation since the actual error covariance matrix cannot be calculated due to the nonlinearity of the system, a condition that can theoretically cause filter divergence.

However, the extended Kalman tilter has been found to work in numerous practical applications, with the only test being whether the filter works in actual use (Gelb, 1974). As demonstrated in Chapter 4, the good results using simulated and actual data support the assumption that the extended Kalman filter is an appropriate choice for this application.

For filter propagation, current estimates of $x(t \mid t-1)$ and $P(t \mid t-1)$ are available from the previous filter iteration. As explained in Section 3.1.1, the governing equations for the system model are:

$$
\underline{\underline{x}}=A \underline{x}+G \underline{w}
$$

and

$$
\Xi=C \underline{x}+D \underline{u} \text {. }
$$

The $D \underline{u}$ term is called the feed-forward term and represents the immediate effect of control inputs on output. As discussed earlier, control inputs are not modeled.

\subsubsection{Update Step}

The first step in the filter propagation is to determine the innovation term $I$, which is defined by the equation:

$$
I=I-(C \times x(t \mid t-1))
$$

This is the difference between the expected measurement vector obtained from the state estimate $x(t+1 \mid t)$ calculated during the previous filter update step and the actual 
measurement vector derived from the vehicle's sensors. Next, the covariance matrix of innovation, $v$, is calculated:

$$
v=C \times P(t \mid t-1) \times C^{\prime}+R,
$$

where $R$ is the error covariance matrix of the measurement vector, $\underline{z}$. The Kalman gain $K$ is then calculated:

$$
K=P(t \mid t-1) \times C^{\prime} \times \operatorname{inv} v(v)
$$

The next step is to calculate the new state vector estimate using the value of $x(t+1 \mid t)$ determined during the previous filter update step:

$$
\underline{x}(t \mid t)=\underline{x}(t \mid t-1)+K \times I
$$

Finally, the new error covariance is computed:

$$
P(t \mid t)=(I d e n t-K \times C) \times P(t \mid t-1) \times(\text { Ident }-K \times C)^{\prime}+K \times R \times K^{\prime}
$$

where Ident in this case is the identity matrix with the same dimensions as $K \times C$.

\subsubsection{Prediction Step}

For the standard Kalman filter, the predicted values of the state vector and the error covariance matrix for the next time step using data up to the present time are now calculated. Since the Kalman filter is implemented as a discrete instead of continuous filter, a conversion of the propagation equations from their continuous forms given in Section 3.1.1 to their discrete counterparts is performed. This transformation uses the MATLAB function "c2d", which takes the $A$ and $G$ matrices and the filter time step as inputs and provides the discrete matrices $\phi$ (translation of the $A$ matrix) and $\Gamma$ (translation of the $G$ matrix) as outputs. Once the conversion to discrete matrices is made, the predictions are computed:

$$
\underline{x}(t+1 \mid t)=\phi \times \underline{I}(t \mid t)+\Gamma \times \underline{w},
$$




$$
P(t+1 \mid t)=\phi \times P(t \mid t) \times \phi^{\prime}+\Gamma \times Q \times \Gamma^{\prime},
$$

where $Q$ is the error covariance matrix for the white noise, $\underline{w}$.

\subsubsection{Implications of the Extended Kalman Filter}

To obtain a new state estimate, the extended Kalman filter uses a first-order Taylor series expansion about the current state estimate to linearize the problem at each update step. In the steady-state version of a linear Kalman filter, the Kalman gain $K$ is a constant. For the extended Kalman filter, it must he recomputed at each update step (Gelb, 1974).

\subsection{Modeling System Noise}

Prior to using the Kalman filter, predictions must be made concerning the estimated errors in the sensors used. All data used by the filter are weighted based on these predicted errors. A measurement considered very accurate has a strong effect on the state estimate. For example, if position information is considered very accurate relative to heading and velocity, the estimates of the latter two are altered from their measured values as necessary to ensure that the estimate of position is close to the measured value. Poor position information places more reliance on heading and velocity measurements. To ensure optimum filter performance, it is essential that the errors be modelled as accurately as possible.

Another important assumption in the Kalman filter is that any errors in the control input vector and the measurement vector are unbiased and follow a Gaussian distribution. For nearly all cases, the assumption of Gaussian errors is valid considering the characteristics of the sensors employed. The prominent exception is the positioning system. While normally the errors can be treated as Gaussian, occasionally the system produces anomalously large errors in position. If these position fixes are treated hy the filter as valid, improper changes in the elements of the state vector are made, resulting in rapid filter degradation. Identifying and ignoring these bad data is an integral part of the modified filter algorithm. 


\subsubsection{Eliminating Non-Gaussian Position System Errors}

The grossly inaccurate position measurements occasionally produced by acoustic positioning systems are frequently referred to as "Alyers". Removing them from the database is vital, but care must be taken. If the allowable tolerance between estimated position and measured position is too tight, valid position fixes can be rejected by the filter. Once this divergence between estimated and actual position occurs, the likelihood of ever accepting another position fix is greatly reduced. This problem is most acute when a long interval exists between fixes. If vehicle heading or velocity is estimated inaccurately during this interval, the difference between estimated and actual vehicle position grows. If the discrepancy becomes large enough, when position information is once again obtained it is considered to be a flyer and rejected. With no further position information, the state estimate of position becomes increasingly inaccurate.

To provide a window that is likely to reject flyers while accepting all valid fixes, the standard allowable error is established at ten times the standard deviation of the position system. To provide for time-dependent system errors, an addition is made to the allowable error based on the time since the last position system fix, given by

Additional allowable error $=0.1 \mathrm{~m} \times \Delta t$,

where

$$
\Delta t=\text { Time since last position fix in seconds. }
$$

Fixes that yield a difference between the current estimated position and the position system fix of greater than this total allowable error are rejected. The method for determining the standard deviation is discussed in Section 3.5.3.2.

The decision to use a cutoff point of ten times the standard deviation is not based on any formula. It is large enough to admit reasonable data, but still should reject any flyers. To help prevent filter divergence between estimated position and valid fix information, a count is maintained within the program of the number of rejected fixes. By monitoring this count during vehicle operations, filter divergence can be recognized by continuous fix rejection. The window of acceptance can then be adjusted as necessary to 
allow the filter to accept new fixes and thus change the state estimates as appropriate to reflect the valid position information.

\subsubsection{Random Forcing Function Noise}

The random forcing function noise is expressed by the error covariance matrix $Q$. It is a $6 \times 6$ matrix since there are six terms that drive the system, three linear accelerations and three angular velocities. It is all zeros except on the main diagonal since each of these - parameters is considered to be independent of the others, making the cross correlation terms zero.

The magnitude of the error covariance terms must be estimated from knowledge of the vehicle dynamics accounting for tow ship motion, tow line motion, and vehicle dynamics. For this filter, the standard deviation of the linear accelerations is set at $0.2 \mathrm{~m} /$

$\mathrm{s}^{2}$, and that of the angular velocimeters at $2 \%$. These constants are incorporated into the KFSetup program.

\subsubsection{Measurement Uncertainty}

The measurement uncertainty is incorporated into the $17 \times 17$ error covariance matrix $R$. The portions of this matrix applicable to each sensor are addressed separately below.

\subsubsection{Linear Accelerometer Uncertainty}

The uncertainties in the linear accelerometer measurements have two sources. One is the noise and bias inherent in any instrument, which can be determined by test or by using the manufacturer's specifications. The other is caused by the displacement of the sensor from the vehicle's reference point for the body-referenced coordinate system.

As shown in Figure 3-3, the vehicle's center of rotation and therefore its most convenient body-coordinate reference point is the point of attachment to the tow line. Assuming the vehicle is not experiencing an actual linear acceleration to the left $(-X$ direction), the $X$-accelerometer would still sense acceleration if angular velocity in the yaw direction (change in heading) were non-zero. This phenomenon is governed by the following coupled equations (Catford, 1978): 


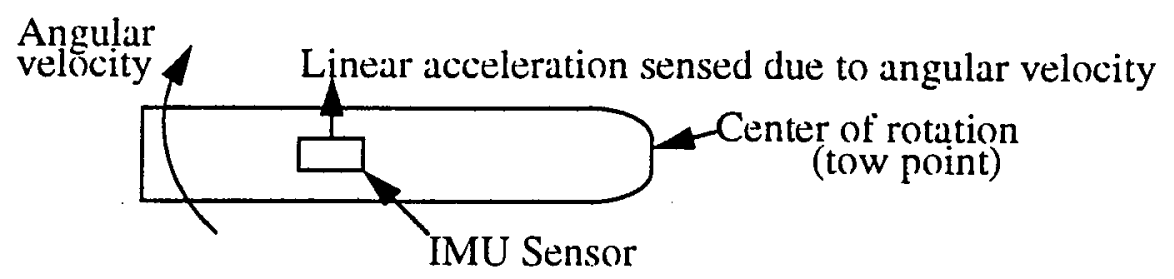

Fig. 3-3. Linear accelerometer output caused by angular velocity.

$$
\begin{gathered}
\alpha_{x}=u+q w-v r+a_{x}\left(-q^{2}-r^{2}\right)+a_{y}(-r+p q)+a_{z}(q+p r)+g \sin \theta \\
\alpha_{y}=v-p w+u r+a_{x}(r+q p)+a_{y}\left(-p^{2}-r^{2}\right)+a_{z}(-p+q r)-g \sin \phi \cos \theta \\
\alpha_{z}=w+p v-q u+a_{x}(-q+r p)+a_{y}(p+r q)+a_{z}\left(-p^{2}-q^{2}\right)-g \cos \theta \cos \phi
\end{gathered}
$$

where $\alpha_{x}, \alpha_{y}$, and $\alpha_{z}$ are the outputs of the accelerometers; $a_{x}, a_{y}$ and $a_{z}$ are actual linear accelerations; $u, v$, and $w$ are velocities; $\phi=$ roll; $\theta=$ pitch; $p, q, r$ are the angular velocities; $g$ is the gravity vector.

To simplify the filter algorithm, this addition to linear acceleration measurements due to angular velocities is ignored. The justification is twofold: first, for a towed vehicle the magnitude of the linear acceleration caused by the angular velocities is small compared to actual linear accelerations; second, over a relatively small time interval, the angular velocities have a mean of zero due to the damped sinusoidal motion of the vehicle. Therefore, any errors induced by this simplification are cancelled out when the angular velocity reverses direction. As a result, the standard deviation for the linear acceleration measurements are set at $0.1 \mathrm{~m} / \mathrm{s}^{2}$.

\subsubsection{Doppler Velocimeter Uncertainty}

As part of its data stream, the doppler velocimeter provides an error velocity. This error velocity is calculated by the velocimeter using a proprietary RDI Instruments program. Factored into this calculation are the depth cell size, the altitude of the yehicle above the bottom, the vehicle velocity, and the number of pings per ensemble $(R D I, 1993)$. 
This error velocity is used by the Kalman filter program as the standard deviation for velocity measurements. Therefore, these elements of the $R$ matrix vary with each filter update step.

\subsubsection{Position System Uncertainty}

The standard deviation for acoustic positioning systems is determined based on operator knowledge of the system and takes into account such parameters as system frequency and distance from the transponder array. It is established prior to filter operation as part of the KFSetup program but can be changed during operations if desired. For other

systems, such as terrain-relative position fixes, the standard deviation can be made part of the data stream hased on the estimated accuracy of the fix and can therefore be varied from fix to fix.

Of all the measurement uncertainties, this requires the greatest attention. If it is assigned to be too large, the Kalman filter does not weight position fixes enough, resulting in potentially significant errors in the estimated position. This can adversely affect the quality of any sidescan-sonar or video data obtained during vehicle operations.

Conversely, if it is set too small there is an increased danger of creating sufficient divergence between the estimated and actual positions to cause invalid position fix rejections.

\subsubsection{Depth Sensor Uncertainty}

When operating in deep water, the depth sensor has one important drawback: because of the limitations of its computer hit capacity, it can only resolve depth to the nearest $0.1 \mathrm{~m}$. Coupled with the normal fluctuations of the instrument, this tends to make the output vary frequently within a $(0.4-\mathrm{m}$ band. Therefore, the standard deviation for . deep-water operations is set at $0.1 \mathrm{~m}$, which places nearly all fluctuations within two standard deviations of the actual value. This standard deviation can be reduced appropriately for shallow-water operations when the resolution is higher. 


\subsubsection{Angular Velocimeter Uncertainty}

Estimation of the angular velocimeter uncertainty is fairly straightforward. Prior testing or manufacturer's specifications are used to determine the expected standard deviation. For the instruments in use, this is set at $0.5 \%$.

\subsubsection{Pitch and Roll Uncertainty}

Like the depth sensor, the pitch and roll sensors are digital and therefore have a noticeable minimum resolution. For these sensors this resolution is $0.1^{\circ}$, and normal fluctuations in a $0.2^{\circ}$ band are observed. To account for unmeasurable biases and to prevent the filter from weighting these measurements too heavily, a standard deviation of $0.5^{\circ}$ is assigned.

\subsubsection{Heading Uncertainty}

Heading is measured directly by the magnetic compass and the gyro. For each, the applicable formula is

True Heading = Indicated Heading - Sensor Bias .

Since the bias for each sensor is modeled within the Kalman filter as a state variable, additional measurements improve the estimate of hias and therehy make the estimate of true heading more accurate.

The standard deviation of the magnetic compass is constant and is set at $1.5^{\circ}$ to account for normal fluctuations. If frequent thruster operations are envisioned, this standard deviation may have to be increased due to the effects of the magnetic fields induced during thruster operations. Due to its greater stability, the gyro has a constant standard deviation set at ().1".

To provide an independent measurement of true heading, which is necessary to correct sensor biases, two different techniques are possible. For a highly maneuverable vehicle operating in a small area, an acoustic transponder can be mounted both at the front and at the back of the vehicle. By comparing the positions of each transponder, true heading can be derived. The standard deviation for this technique depends on the accuracy of the positioning system used. 


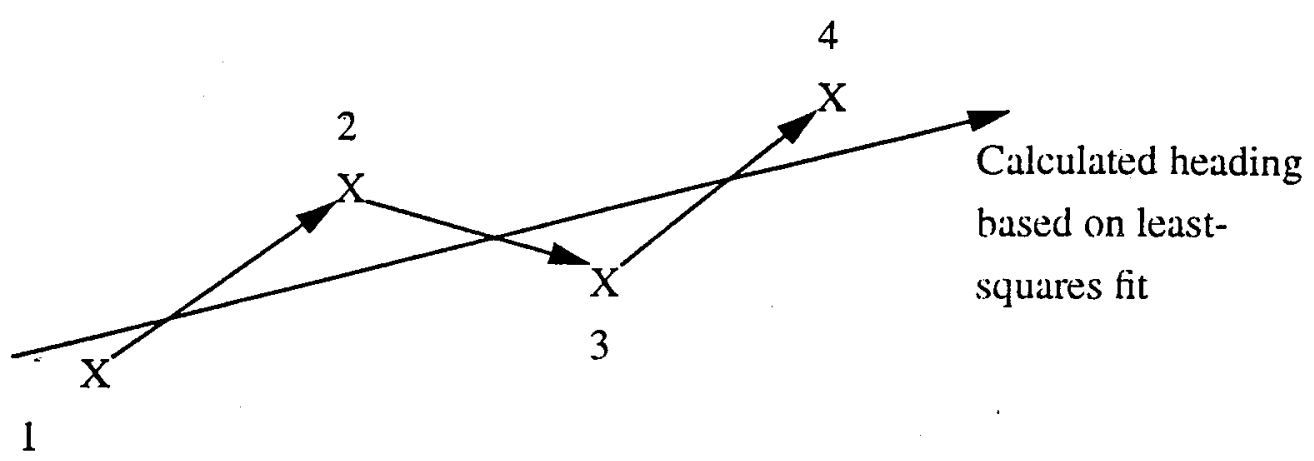

Fig. 3-4. Calculation of true heading and standard deviation.

For a towed vehicle, vehicle motion can be assumed to be nearly entirely in the direction of the vehicle's front. Therefore, a measurement of true heading is obtained by calculating a least-squares fit to the last four position fixes. Although the standard deviation of this heading measurement is difficult to calculate precisely, an approximation is derived as follows (see Fig. 3-4).

After obtaining the least-squares solution, a separate heading measurement is made between each adjacent pair of fixes ( 1 and 2, 2 and 3,3 and 4). The difference between each of these heading measurements and the overall least-squares measurement is averaged. This average difference in heading measurements is considered to be the standard deviation of the measurement. Although this is not precise, it has the desired property of providing less standard deviation as the fix measurements become less scattered from the best-fit track.

\subsection{Establishing Reference Points and Initial Conditions}

\subsubsection{Reference Points}

To ensure correct matrix manipulations while avoiding round-off errors, MATLAB works better with well-conditioned matrices, which means that the elements of the matrices are as close together as possible in terms of order of magnitude. To achieve this conditioning, reference points for position and time must be established. These reference values are subtracted from the actual values prior to use in the filter. The filter uses only 
the difference between the reference values and the current values, resulting in numbers smaller by several orders of magnitude.

For X-and Y-positions, the Universal Transverse Mercator (UTM) system is used. This system divides the oceans into rectangular grids with scales in meters. A suitable reference point is chosen in the area of vehicle operations and included in the KFSetup program:

The Z-position reference point is dependent on expected vehicle operating depth. To achieve a right-handed coordinate system, the filter actually uses vehicle altitude above or below the reference point rather than depth, but this coordinate system change is imbedded within the filter algorithm. A reference depth near the expected operating depth is chosen by the operator in the KFSetup program prior to commencing operations.

The final reference point is time. Each sensor package data stream contains a time stamp with units of Julian days. The reference time for each day is the beginning of the Julian day. Therefore, the time value used in the filter is always a fraction of a day.

\subsubsection{Initial State Vector and Error Covariance Matrix}

Prior to the first filter undate step, the initial state vector $x$ and error covariance matrix $P$ must be provided. Normally, the initial sensor data obtained are used for the state vector, and the predetermined $R$ matrix is augmented with the initial calculated variable elements of $R$ (for velocimeter and heading measurements) to provide $P$. However, if some other more accurate method is available for determining any of these variables, the better value can be substituted instead.

\subsubsection{Removing Known Biases}

Of crucial importance in optimizing Kalman filter performance is the removal of bias. Uncorrected bias provides a consistent source of error in the state estimate vector and must be eliminated as much as possible.

Because of its use of doppler to measure velocity, whereby zero frequency change equates to zero velocity in that direction, the doppler velocimeter is relatively immune to hias. Therefore, no attempt is made to compensate for it. 
The position information is also insensitive to bias, since the use of a reference point automatically removes hias from the system. Once again, no bias correction is needed.

The bias of the pitch and roll sensors is directly related to the precision of their placement on the vehicle. Normally, the final installation of the sensors on the vehicle is performed in the lab, which allows the sensors to be checked to ensure they are unbiased. Therefore, no bias adjustment is made in the filter. If the sensors must be replaced at sea, accurate measurement of pitch and roll on a moving ship is virtually impossible, so there is no way to measure bias accurately. In this case, the sensor is mounted as precisely as possible and the filter assumes unbiased measurements. Since pitch and roll are usually rather small, the effects of undetermined bias on the coordinate transformations is negligible.

Compensation for gyro hias and magnetic compass bias is discussed in Section 3.5.3.5. Additionally, any known initial bias is included as the initial condition of the state variable for each heading sensor hias. Short-term effects on magnetic compass bias caused by thruster operation cannot be calculated and are therefore ignored.

As discussed in Section 3.5.2, the biases inherent to the linear accelerometers and angular velocimeters must be determined by laboratory testing. With the vehicle motionless and with zero pitch and roll, the output voltage of each instrument can be measured. The voltage caused by the gravity vector is calculated so as not to be removed from the instrument measuring vertical acceleration, but the other measured voltages are subtracted from the data. These biases are also included in the KFSetup program.

\subsection{Changes Required to the Standard Kalman Filter}

In this application, there are three major changes that must be made to the standard Kalman filter described in Section 3.4. First, due to the variability of the data update rate, a constant filter update frequency is impractical. Second, the difference in coordinate systems among the various sensors must be addressed. Finally, there are gaps in certain data streams, especially for $\mathrm{X}$ - and Y-position fixes. The filter must be able to function despite these gaps to provide continuous state estimates. 


\subsubsection{Adjustments Required Due to Varying Filter Update Rate}

\subsubsection{Determining the Desired Time Step}

To determine the desired time step, competing objectives must be considered. In support of a longer time step, there are two major arguments. First, adequate time must be given to obtain the data necessary for the propagation of the filter. Second, the interval must be long enough to permit real-time processing of the data.

There is only one counterpoint in support of a shorter time step, but it is a vital one. If the time step is too long, the true vehicle dynamics will be lost, especially if significant maneuvering is occurring. Although a long time step may provide a reasonable approximation of average vehicle position and attitude, the lack of precise, continuous information will degrade any sidescan-sonar or video imaging accuracy. A compromise is necessary.

Of the four data packages described in Section 3.2, only two have update rates that are both consistent and rapid. The IMU package produces data at approximately $10 \mathrm{~Hz}$, while the attitude package operates at ahout $5 \mathrm{~Hz}$. Therefore, the Kalman filter is designed with a minimum requirement of having data from these two packages prior to performing a filter update step. As an overall compromise, a time step of $0.5 \mathrm{~s}$ is used. This is short enough to capture the system dynamics, but long enough to allow real-time processing of data and to ensure both IMU and attitude package information is normally available.

To prevent filter processing error in the event of an interruption in data flow, the filter algorithm checks to ensure that at least one sample of each data package is available within the $0.5 \mathrm{~s}$ of data to he processed. If either is missing, the filter continues to accept new data until both are present. As soon as this condition is met, the data obtained from each sensor during that time step are averaged and the next filter update step is performed.

\subsubsection{Predicting the Next State Vector and Error Covariance Matrix}

In the standard Kalman filter, the last step of filter propagation produces the predicted values of $\underline{x}(t+1 \mid t)$ and $P(t+1 \mid t)$. As described in Section 3.4.2.2, one of the inputs required for the conversion from a continuous to a discrete matrix is the magnitude of the time step. For this filter, that magnitude is unknown. First, it will probably not be exactly $0.5 \mathrm{~s}$, since the actual time step is the difference in time between the first and the 
last data received prior to $0.5 \mathrm{~s}$ elapsing. Second, if either IMU package or attitude package data is not received during that first (0.5-s interval, the filter algorithm waits until the missing data are provided. Thus, the time interval could be considerably longer.

To correct for these varying time steps, a small change in filter mechanics is required. Instead of computing the predicted values at the end of a given filter update step, the filter algorithm waits until the beginning of the next filter undate cycle. Therefore, the filter time step is known since the algorithm has already allowed $0.5 \mathrm{~s}$ to elapse and has then checked for the two data streams, waiting as necessary to obtain them. By allowing the predicted values to lag until the next time step is confirmed, the continuous-to-discrete conversion can be made accurately.

\subsubsection{Coordinate System Transformations}

Due to the difference in coordinate systems among the various sensors, two separate calculations are required. Both use the current estimates of roll, pitch, and heading as input. The first removes the effect of the gravity vector on the linear accelerometer measurements. The second computes the current $A$ matrix used in the prediction of the next state estimate and error covariance matrix.

\subsubsection{Removing the Effect of the Gravity Vector}

To provide an accurate reflection of vehicle linear acceleration, the output of the IMU package must be modified to remove the gravity vector bias. This vectorg is assumed constant with a value of $9.8 \mathrm{~m} / \mathrm{s}^{2}$ pointing in the $-z$ direction in earth-referenced coordinates. The filter algorithm uses the current state estimates of roll and pitch to calculate the component of the gravity vector included in the output of each of the three linear accelerometers. The effect of the gravity vector on $x$-acceleration is shown in Fig. 3-5 as an example. The calculations are as follows ( $\phi=$ roll, $\omega=$ pitch, $g=-9.8$ ):

Actual $x$-acceleration $=($ Measured $x$-acceleration $)+g \sin \phi$,

Actual y-acceleration $=($ Measured y-acceleration $)-g \sin \omega$,

Actual z-acceleration $=($ Measured z-acceleration $)-g \cos \phi \cos \omega$. 


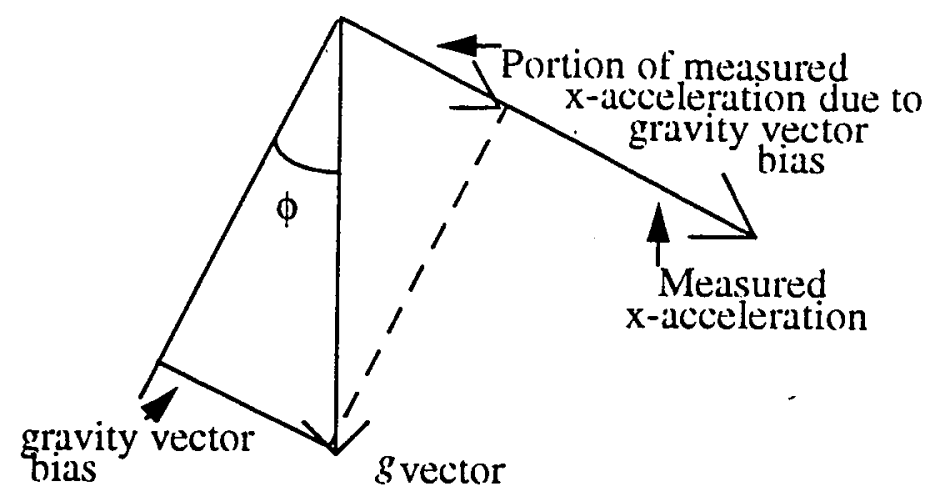

Fig. 3-5. Effect of gravity vector on measured $\mathrm{x}$-acceleration.

These calculations are actually approximations because the effect of one attitude on the measurement of another is ignored. For example, as pitch increases the sensitivity of the roll sensor is decreased due to the lessened gravity vector component on the inclinometer's sensitive axis. In the worst case, a pitch of 90 degrees results in no sensitivity to roll. For typical vehicle operations, during which pitch and roll are normally less than 10 degrees, the errors introduced by these approximations are negligible.

\subsubsection{Calculating the $A$ Matrix}

As explained in Section 3.4.2.2, part of the prediction step of the filter update cycle converts the continuous time $A$ and $G$ matrices into their discrete time counterparts. Unlike the linear filter case, in this nonlinear filter the $A$ matrix must be recalculated during each prediction step.

To be more specific, the detailed version of the system propagation equation is as follows. The $A$ matrix is constant with the exception of the $3 \times 3$ portion identified with " $\alpha$," because this portion of the $A$ matrix propagates the change in vehicle position due to the previously estimated vehicle velocities. Since vehicle velocities are body-referenced and positions are earth-referenced, a coordinate transformation is necessary.

The current heading estimate, which is maintained in the traditional clockwisepositive system used by the compass and gyro, is altered to a counterclockwise-positive yaw angle to maintain a right-handed coordinate system. With units of radians, this yaw angle is calculated by: 


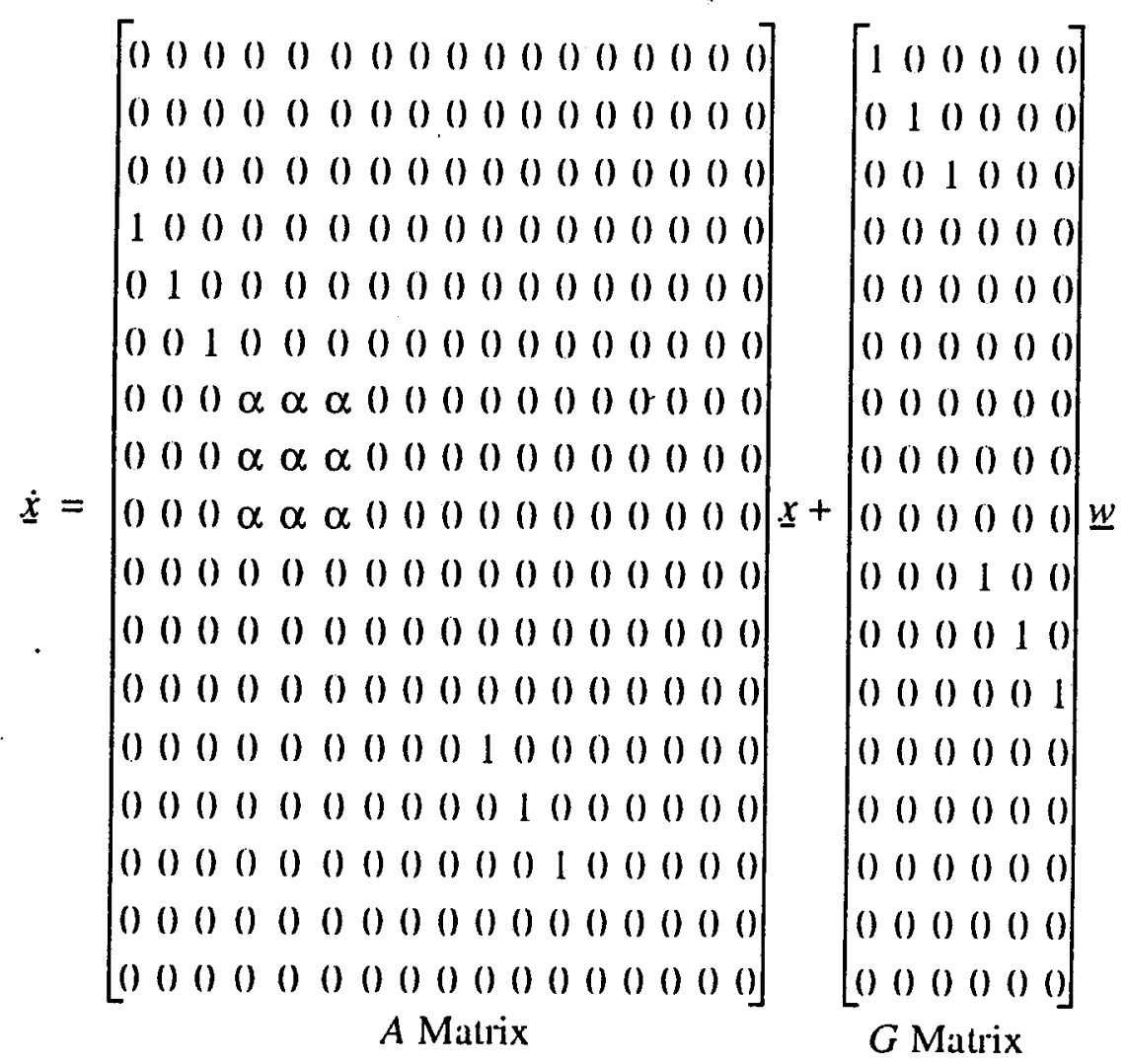

$$
\kappa=2 \pi-(\text { Current Heading Estimate }) .
$$

The $3 \times 3$ nonzero matrix within the $A$ matrix is computed as follows. Required inputs are the current state estimates of roll $(\phi)$, pitch $(\omega)$, and heading $(\kappa)$.

Element $(1,1)=\cos \phi \cos \kappa$

Element $(1,2)=\sin \omega \sin \phi \cos \kappa-\cos \omega \sin \kappa$

Element $(1,3)=\cos \omega \sin \phi \cos \kappa+\sin \omega \sin \kappa$

Element $(2,1)=\sin \kappa \cos \phi$

Element $(2,2)=\sin \omega \sin \phi \sin \kappa+\cos \omega \cos \kappa$

Element $(2,3)=\cos \omega \sin \phi \sin \kappa-\sin \omega \cos \kappa$

Element $(3,1)=-\sin \phi$

Element $(3,2)=\sin \omega \cos \phi$

Element $(3,3)=\cos \omega \cos \phi$ 


\subsubsection{Varying the $C$ Matrix with the Available Data}

Section 3.7.1.1 describes the process by which the filter ensures it has both IMU and attitude information available prior to performing an update step. During this period of accepting new data, doppler velocimeter and/or X-Y position information may also be obtained. To perform the update step correctly in light of the available data, the filter algorithm keeps track of which data are available prior to performing the update operation. After averaging all data from each sensor, the $C$ matrix must be computed for use by the filter. As part of this computation, the measurement vector $z$ is also determined based on which sets of data have been obtained.

After the first four position tixes have been obtained (and therefore true heading information is available with each subsequent fix), there are four possibilities for the $C$ matrix and measurement vector:

1. Only IMU package and attitude package data are available. The measurement vector is:

$$
\Xi=\left[\begin{array}{c}
\text { x-acceleration } \\
\text { y-acceleration } \\
\text { z-acceleration } \\
z \text {-position } \\
\text { roll rate } \\
\text { pitch rate } \\
\text { yaw rate } \\
\text { roll } \\
\text { pitch } \\
\text { heading by gyro } \\
\text { heading hy compass }
\end{array}\right] \text {, }
$$

and the corresponding $C$ matrix is: 


$$
C=\left[\begin{array}{ccccccccccccccccc}
1 & 0 & 0 & 0 & 0 & 0 & 0 & 0 & 0 & 0 & 0 & 0 & 0 & 0 & 0 & 0 & 0 \\
0 & 1 & 0 & 0 & 0 & 0 & 0 & 0 & 0 & 0 & 0 & 0 & 0 & 0 & 0 & 0 & 0 \\
0 & 0 & 1 & 0 & 0 & 0 & 0 & 0 & 0 & 0 & 0 & 0 & 0 & 0 & 0 & 0 & 0 \\
0 & 0 & 0 & 0 & 0 & 0 & 0 & 0 & 1 & 0 & 0 & 0 & 0 & 0 & 0 & 0 & 0 \\
0 & 0 & 0 & 0 & 0 & 0 & 0 & 0 & 0 & 1 & 0 & 0 & 0 & 0 & 0 & 0 & 0 \\
0 & 0 & 0 & 0 & 0 & 0 & 0 & 0 & 0 & 0 & 1 & 0 & 0 & 0 & 0 & 0 & 0 \\
0 & 0 & 0 & 0 & 0 & 0 & 0 & 0 & 0 & 0 & 0 & 1 & 0 & 0 & 0 & 0 & 0 \\
0 & 0 & 0 & 0 & 0 & 0 & 0 & 0 & 0 & 0 & 0 & 0 & 1 & 0 & 0 & 0 & 0 \\
0 & 0 & 0 & 0 & 0 & 0 & 0 & 0 & 0 & 0 & 0 & 0 & 0 & 1 & 0 & 0 & 0 \\
0 & 0 & 0 & 0 & 0 & 0 & 0 & 0 & 0 & 0 & 0 & 0 & 0 & 0 & 1 & -1 & 0 \\
0 & 0 & 0 & 0 & 0 & 0 & 0 & 0 & 0 & 0 & 0 & 0 & 0 & 0 & 1 & 0 & -1
\end{array}\right],
$$

reflecting the position in the state estimate vector $x$ of $\mathrm{x}$-acceleration $(\mathrm{x} 1)$, $\mathrm{y}$-acceleration (x2), z-acceleration (x3), z-position ( $x 9)$, roll rate $(x 11)$, pitch rate $(x 11)$, yaw rate (x12), roll (x13), pitch (x14), heading (x15), gyro bias (x16), and compass bias (x17).

2. Both attitude package and $X Y$ position information are available but no doppler velocimeter data. The measurement vector is:

$$
z=\left[\begin{array}{c}
x \text {-acceleration } \\
y \text {-acceleration } \\
z \text {-acceleration } \\
\text { x-position } \\
y \text {-position } \\
\text { z-position } \\
\text { roll rate } \\
\text { pitch rate } \\
\text { yaw rate } \\
\text { roll } \\
\text { pitch } \\
\text { heading by gyro } \\
\text { heading by compass } \\
\text { true heading }
\end{array}\right] \text {, }
$$

and the corresponding $C$ matrix is: 


$$
C=\left[\begin{array}{rrrrrrrrrrrrrrrrr}
1 & 0 & 0 & 0 & 0 & 0 & 0 & 0 & 0 & 0 & 0 & 0 & 0 & 0 & 0 & 0 & 0 \\
0 & 1 & 0 & 0 & 0 & 0 & 0 & 0 & 0 & 0 & 0 & 0 & 0 & 0 & 0 & 0 & 0 \\
0 & 0 & 1 & 0 & 0 & 0 & 0 & 0 & 0 & 0 & 0 & 0 & 0 & 0 & 0 & 0 & 0 \\
0 & 0 & 0 & 0 & 0 & 0 & 1 & 0 & 0 & 0 & 0 & 0 & 0 & 0 & 0 & 0 & 0 \\
0 & 0 & 0 & 0 & 0 & 0 & 0 & 1 & 0 & 0 & 0 & 0 & 0 & 0 & 0 & 0 & 0 \\
0 & 0 & 0 & 0 & 0 & 0 & 0 & 0 & 1 & 0 & 0 & 0 & 0 & 0 & 0 & 0 & 0 \\
0 & 0 & 0 & 0 & 0 & 0 & 0 & 0 & 0 & 1 & 0 & 0 & 0 & 0 & 0 & 0 & 0 \\
0 & 0 & 0 & 0 & 0 & 0 & 0 & 0 & 0 & 0 & 1 & 0 & 0 & 0 & 0 & 0 & 0 \\
0 & 0 & 0 & 0 & 0 & 0 & 0 & 0 & 0 & 0 & 0 & 1 & 0 & 0 & 0 & 0 & 0 \\
0 & 0 & 0 & 0 & 0 & 0 & 0 & 0 & 0 & 0 & 0 & 0 & 1 & 0 & 0 & 0 & 0 \\
0 & 0 & 0 & 0 & 0 & 0 & 0 & 0 & 0 & 0 & 0 & 0 & 0 & 1 & 0 & 0 & 0 \\
0 & 0 & 0 & 0 & 0 & 0 & 0 & 0 & 0 & 0 & 0 & 0 & 0 & 0 & 1 & -1 & 0 \\
0 & 0 & 0 & 0 & 0 & 0 & 0 & 0 & 0 & 0 & 0 & 0 & 0 & 0 & 1 & 0 & -1 \\
0 & 0 & 0 & 0 & 0 & 0 & 0 & 0 & 0 & 0 & 0 & 0 & 0 & 0 & 1 & 0 & 0
\end{array}\right] .
$$

3. Both attitude package and doppler velocimeter data are available but no XY position information. The measurement vector is:

$$
z=\left[\begin{array}{c}
x \text {-acceleration } \\
y \text {-acceleration } \\
z \text {-acceleration } \\
\text { x-velocity } \\
\text { y-velocity } \\
\text { z-velocity } \\
\text { z-position } \\
\text { roll rate } \\
\text { pitch rate } \\
\text { yaw rate } \\
\text { roll } \\
\text { pitch } \\
\text { heading by gyro } \\
\text { heading by compass }
\end{array}\right]
$$

and the corresponding $C$ matrix is: 


$$
C=\left[\begin{array}{lllllllllllllllll}
1 & 0 & 0 & 0 & 0 & 0 & 0 & 0 & 0 & 0 & 0 & 0 & 0 & 0 & 0 & 0 & 0 \\
0 & 1 & 0 & 0 & 0 & 0 & 0 & 0 & 0 & 0 & 0 & 0 & 0 & 0 & 0 & 0 & 0 \\
0 & 0 & 1 & 0 & 0 & 0 & 0 & 0 & 0 & 0 & 0 & 0 & 0 & 0 & 0 & 0 & 0 \\
0 & 0 & 0 & 1 & 0 & 0 & 0 & 0 & 0 & 0 & 0 & 0 & 0 & 0 & 0 & 0 & 0 \\
0 & 0 & 0 & 0 & 1 & 0 & 0 & 0 & 0 & 0 & 0 & 0 & 0 & 0 & 0 & 0 & 0 \\
0 & 0 & 0 & 0 & 0 & 1 & 0 & 0 & 0 & 0 & 0 & 0 & 0 & 0 & 0 & 0 & 0 \\
0 & 0 & 0 & 0 & 0 & 0 & 0 & 0 & 1 & 0 & 0 & 0 & 0 & 0 & 0 & 0 & 0 \\
0 & 0 & 0 & 0 & 0 & 0 & 0 & 0 & 0 & 1 & 0 & 0 & 0 & 0 & 0 & 0 & 0 \\
0 & 0 & 0 & 0 & 0 & 0 & 0 & 0 & 0 & 0 & 1 & 0 & 0 & 0 & 0 & 0 & 0 \\
0 & 0 & 0 & 0 & 0 & 0 & 0 & 0 & 0 & 0 & 0 & 1 & 0 & 0 & 0 & 0 & 0 \\
0 & 0 & 0 & 0 & 0 & 0 & 0 & 0 & 0 & 0 & 0 & 0 & 1 & 0 & 0 & 0 & 0 \\
0 & 0 & 0 & 0 & 0 & 0 & 0 & 0 & 0 & 0 & 0 & 0 & 0 & 1 & 0 & 0 & 0 \\
0 & 0 & 0 & 0 & 0 & 0 & 0 & 0 & 0 & 0 & 0 & 0 & 0 & 0 & 1 & -1 & 0 \\
0 & 0 & 0 & 0 & 0 & 0 & 0 & 0 & 0 & 0 & 0 & 0 & 0 & 0 & 1 & 0 & -1
\end{array}\right] .
$$

4. The final case is when data are available from all sensors. The measurement vector uses the same construction as the state vector.

$$
z=\left[\begin{array}{c}
\text { x-acceleration } \\
y \text {-acceleration } \\
\text { z-acceleration } \\
\text { x-velocity } \\
\text { y-velocity } \\
\text { z-velocity } \\
\text { x-position } \\
\text { y-position } \\
\text { z-position } \\
\text { roll rate } \\
\text { pitch rate } \\
\text { yaw rate } \\
\text { roll } \\
\text { pitch } \\
\text { heading by gyro } \\
\text { heading by compass } \\
\text { true heading }
\end{array}\right] \text {, }
$$

while the $C$ matrix is: 


$$
C=\left[\begin{array}{lllllllllllllllll}
1 & 0 & 0 & 0 & 0 & 0 & 0 & 0 & 0 & 0 & 0 & 0 & 0 & 0 & 0 & 0 & 0 \\
0 & 1 & 0 & 0 & 0 & 0 & 0 & 0 & 0 & 0 & 0 & 0 & 0 & 0 & 0 & 0 & 0 \\
0 & 0 & 1 & 0 & 0 & 0 & 0 & 0 & 0 & 0 & 0 & 0 & 0 & 0 & 0 & 0 & 0 \\
0 & 0 & 0 & 1 & 0 & 0 & 0 & 0 & 0 & 0 & 0 & 0 & 0 & 0 & 0 & 0 & 0 \\
0 & 0 & 0 & 0 & 1 & 0 & 0 & 0 & 0 & 0 & 0 & 0 & 0 & 0 & 0 & 0 & 0 \\
0 & 0 & 0 & 0 & 0 & 1 & 0 & 0 & 0 & 0 & 0 & 0 & 0 & 0 & 0 & 0 & 0 \\
0 & 0 & 0 & 0 & 0 & 0 & 1 & 0 & 0 & 0 & 0 & 0 & 0 & 0 & 0 & 0 & 0 \\
0 & 0 & 0 & 0 & 0 & 0 & 0 & 1 & 0 & 0 & 0 & 0 & 0 & 0 & 0 & 0 & 0 \\
0 & 0 & 0 & 0 & 0 & 0 & 0 & 0 & 1 & 0 & 0 & 0 & 0 & 0 & 0 & 0 & 0 \\
0 & 0 & 0 & 0 & 0 & 0 & 0 & 0 & 0 & 1 & 0 & 0 & 0 & 0 & 0 & 0 & 0 \\
0 & 0 & 0 & 0 & 0 & 0 & 0 & 0 & 0 & 0 & 1 & 0 & 0 & 0 & 0 & 0 & 0 \\
0 & 0 & 0 & 0 & 0 & 0 & 0 & 0 & 0 & 0 & 0 & 1 & 0 & 0 & 0 & 0 & 0 \\
0 & 0 & 0 & 0 & 0 & 0 & 0 & 0 & 0 & 0 & 0 & 0 & 1 & 0 & 0 & 0 & 0 \\
0 & 0 & 0 & 0 & 0 & 0 & 0 & 0 & 0 & 0 & 0 & 0 & 0 & 1 & 0 & 0 & 0 \\
0 & 0 & 0 & 0 & 0 & 0 & 0 & 0 & 0 & 0 & 0 & 0 & 0 & 0 & 1 & -1 & 0 \\
0 & 0 & 0 & 0 & 0 & 0 & 0 & 0 & 0 & 0 & 0 & 0 & 0 & 0 & 1 & 0 & -1 \\
0 & 0 & 0 & 0 & 0 & 0 & 0 & 0 & 0 & 0 & 0 & 0 & 0 & 0 & 1 & 0 & 0
\end{array}\right] .
$$

By using this varying $C$ matrix, the Kalman filter mechanics remain the same for each update step regardless of the available data. Additionally, the error covariance matrix automatically reflects the data available. For each filter update step, each state variable's error covariance is increased due to elapsed time, and then decreased if a measurement of that state variable is available. If a given measurement is not available, the corresponding state estimate has a greater error covariance than before. Similarly, available data reduces the error covariance appropriately based on the assigned standard deviation of that particular sensor output.

\subsubsection{Incorporating Fading Memory}

One problem with the standard Kalman implementation is filter "laziness". In a completely observable system, the Kalman gain approaches zero as time goes to infinity. Therefore, the filter ignores new data since it helieves that it has essentially perfect knowledge of all state variables. Changes in those variables caused by vehicle operations, such as a change in course or velocity, are not reflected in the filter. 
Gelb provides a simple solution to this problem using recursive fading memory filtering. To force the filter to continue using new data, the error covariance of the predicted state vector is artificially increased (Gelb, 1974). This is accomplished with a simple addition to the algorithm for calculating $P(t+1 \mid t)$. Now, the equation is:

$$
P(t+1 \mid t)=s \times \phi \times P(t \mid t) \times \phi^{\prime}+\Gamma \times Q \times \Gamma^{\prime},
$$

with the only change being the addition of the $s$ term, which is the fade factor. This is calculated by

$$
s=\exp (\Delta t / \tau)
$$

where

$\Delta t$ is the measurement interval ( $(0.5 \mathrm{~s})$,

$\tau$ is the age-weighting time constant.

For this filter, the time constant is chosen to be $30 \mathrm{~s}$ to ensure a filter responsive to rapid changes in vehicle parameters while still retaining sufficient memory. The resulting fade factor is 1.0168 .

As Sorenson and Sacks explain (Sorenson and Sacks, 1971), the use of this fade factor does not affect the stability of the Kalman filter. The only disadvantage is that by using the fade factor, the error covariance matrix is altered. Therefore, the error covariance matrix is no longer the best estimate of the error in the state estimate but only an approximation.

\subsection{Observability}

If a system is completely observable, the state vector can be entirely determined from the measurements. If the system is only partially observable, then any system errors involving unobservable quantities that propagate through the filter cannot be identified by measurements. For example, if position is not observed, then any consistent errors in velocity measurements will continue to increase the position error. In contrast, if velocity is not measured but position is, velocity can still be observed due to its known effect on 
position. In the context of vehicle navigation, a partially observable system means that there is no way to provide a reasonable estimate of the unobservable quantities. If that is the case, the filter will be useless.

To be observable, the matrix $\left[C^{T}\left|A^{T} C^{T}\right|\left(A^{T}\right)^{2} C^{T}\right.$ l. . $\left.\mid\left(A^{T}\right)^{n-1} C^{T}\right]$ must have rank $n$ (Gelb, 1974). When all data are available, the $C^{T}$ matrix has 17 linearly independent columns by inspection, and the system is trivially observable. However, when fewer data are available the observability changes. When only IMU package, attitude package, and doppler.velocimeter information is available, the observability matrix is no longer trivial. Powers of $A^{T}$ greater than two yield only zero matrices and do not contribute to observability. Eliminating zero columns and repeated columns,

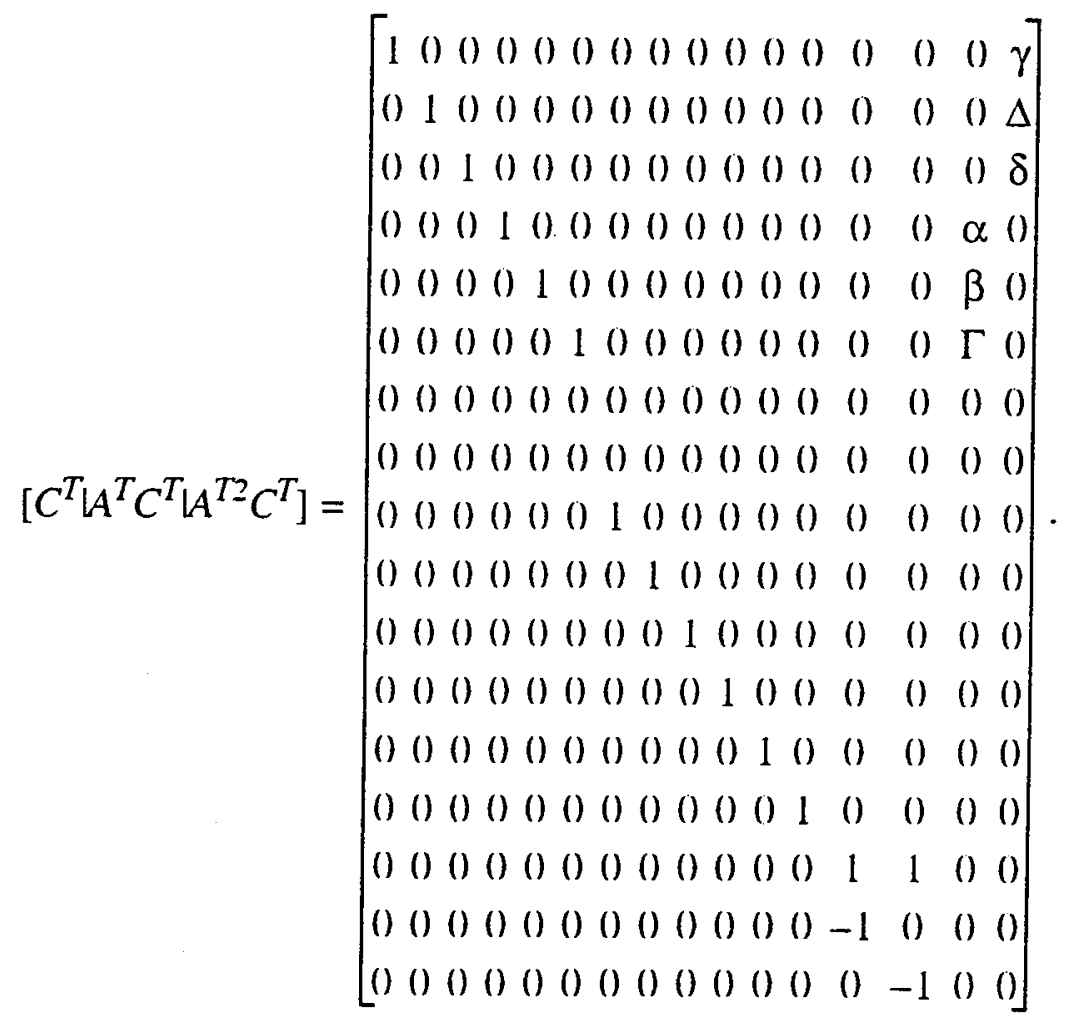

The Greek letters $\alpha, \beta, \Gamma, \gamma, \Delta$, and $\delta$ represent elements of the $3 \times 3$ coordinate transformation matrix. In this case, they do not add to the rank of the matrix since column 15 containing them is simply a linear combination of columns four through six, while column 16 is a linear combination of the first three columns. Therefore, the rank of the matrix is only 14 , leaving three state variahles as unobservahle. Not surprisingly, two of 
the unobservable states in this case are $\mathrm{X}$ - and Y-positions. Without observing position, there is no way to determine if the estimates of velocity and attitude contain errors causing divergence of the state estimates from the actual values. The third unobservable state concerns gyro and compass bias. Without position measurements, true heading measurements are impossible. While independent measurements of gyro and magnetic compass headings allow the filter to observe the difference between the two biases, without true heading measurements the actual value of either cannot be determined (since the difference can be calculated, knowing one of these biases would permit calculation of the other. Thus there is only one additional unobservable state due to the lack of true heading information).

Similar calculations can be performed for the other two possible data combinations. Lacking only velocity data, the system is observable since position measurements can verify the accuracy of velocity estimates. Without X-and Y-position information available at least occasionally, the filter cannot be expected to provide valid estimates. 


\section{Chapter 4}

\section{Kalman Filter Performance Using Simulated Data}

\subsection{Purpose of Simulation}

The initial tests of this Kalman filter are performed using simulated data. This allows simple modification of data parameters to evaluate filter performance under a variety of conditions. Several tests are performed using the same basic data set. The specific parameters for each test are explained in detail.

\subsection{Data Generation}

\subsubsection{Method of Data Generation}

The total time of the simulation is fixed at one hour. This provides ample time for filter evaluation and coincides with the period used for the actual data evaluation conducted in Chapter 5.

Generating a valid data set is a multistep process. First, the control inputs are determined using reasonable values for linear accelerations and angular velocities. Then the nominal standard deviations for the control input measurements and system measurements are computed, along with system initial conditions. These use the values determined in Section 3.5, unless otherwise noted. For all simulated data, initial conditions are found in Table 4-1.

To generate noise-free measurements in the appropriate coordinate system for each sensor, first the attitude measurements are computed for the entire hour using the MATLAB function "dlsim," with the angular velocity measurements as input. Using these noise-free attitude computations, the vehicle-referenced linear accelerations are transformed into earth-referenced coordinates using the coordinate transformation found in Section 3.7.2.2. Next, "dlsim" is used again to provide earth-referenced velocities and 
Table 4-1: Initial Conditions for Simulations

\begin{tabular}{|c|c|}
\hline Parameter & Initial Value \\
\hline X-Velocity & 0 \\
\hline Y-Velocity & $0.5 \mathrm{~m} / \mathrm{s}$ \\
\hline Z-Velocity & 0 \\
\hline X-Position & 0 \\
\hline Y-Position & 0 \\
\hline Z-Position & 0 \\
\hline Roll & 0 \\
\hline Pitch & 0 \\
\hline Heading & $300^{\circ}$ \\
\hline Gyro Bias & 0 \\
\hline Compass Bias & $-18^{\circ}$ \\
\hline
\end{tabular}

positions. Finally, attitude is used to transform the computed velocities into the vehiclereferenced coordinates used in the filter.

After these noise-free measurements are computed, noise is added using the MATLAB function "randn," which provides a Gaussian distribution with the desired variance. Additionally, the frequency of data output from each sensor is determined. The last step is to add desired gyro bias, compass bias, and any gyro drift.

\subsubsection{Noise-free Data Used for All Simulation Tests}

To provide a convenient basis for comparison among the tests, the same noise-free data set is used for each. Figures 4-1 through 4-4 provide a graphic display of a sample of these measurements. 


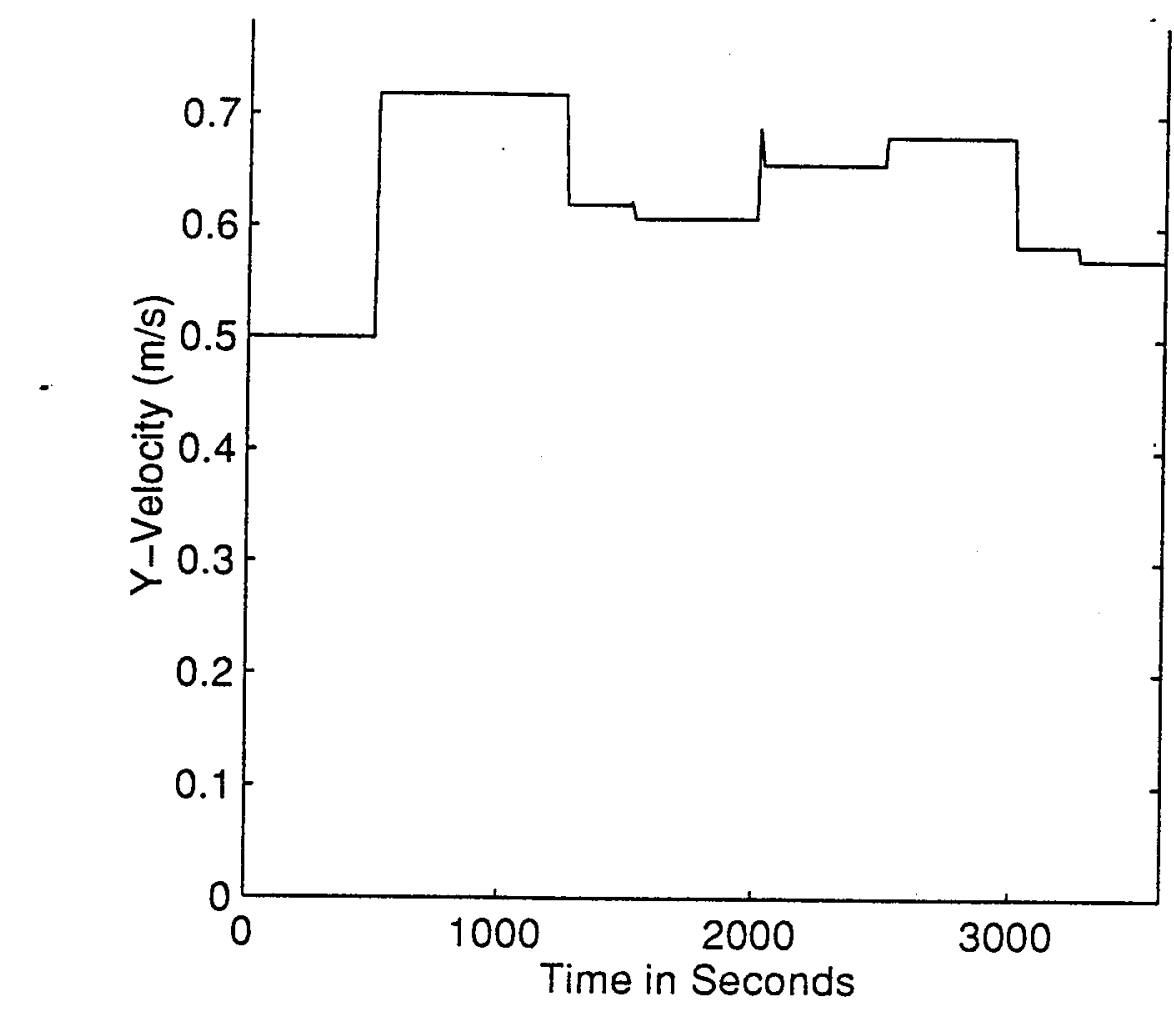

Fig. 4-1. Noise-free measurement of Y-velocity.

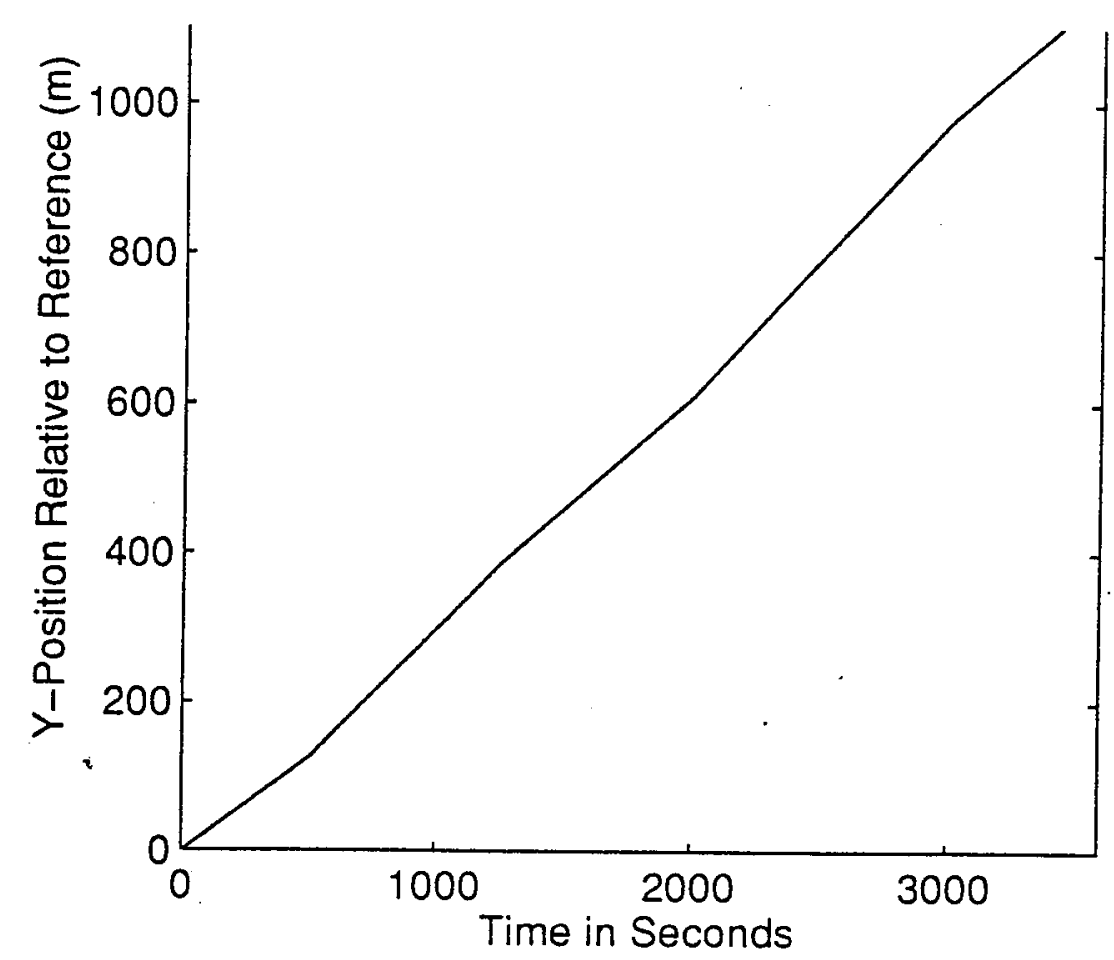

Fig. 4.2. Noise-free measurement of Y-position. 


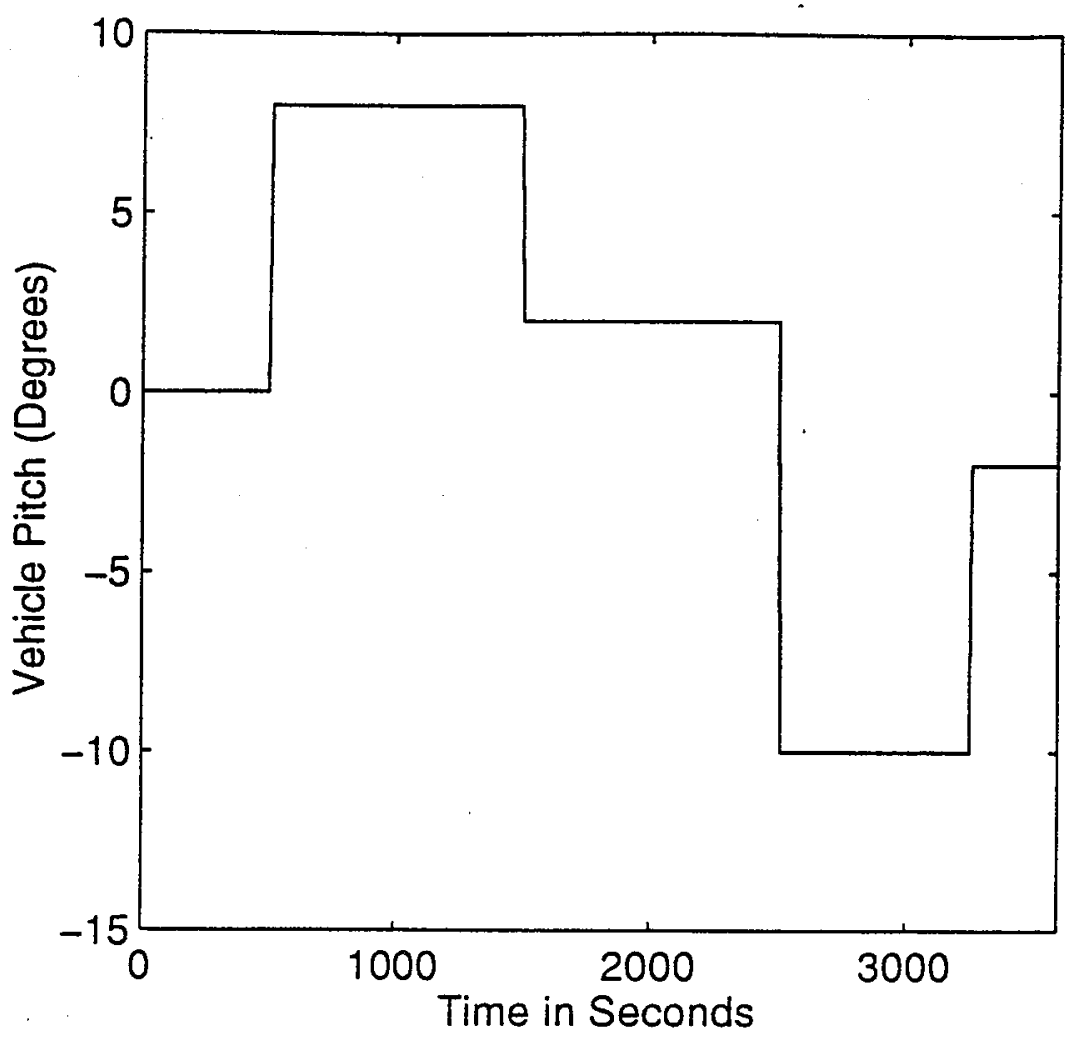

Fig. 4-3. Noise-free measurement of vehicle pitch.

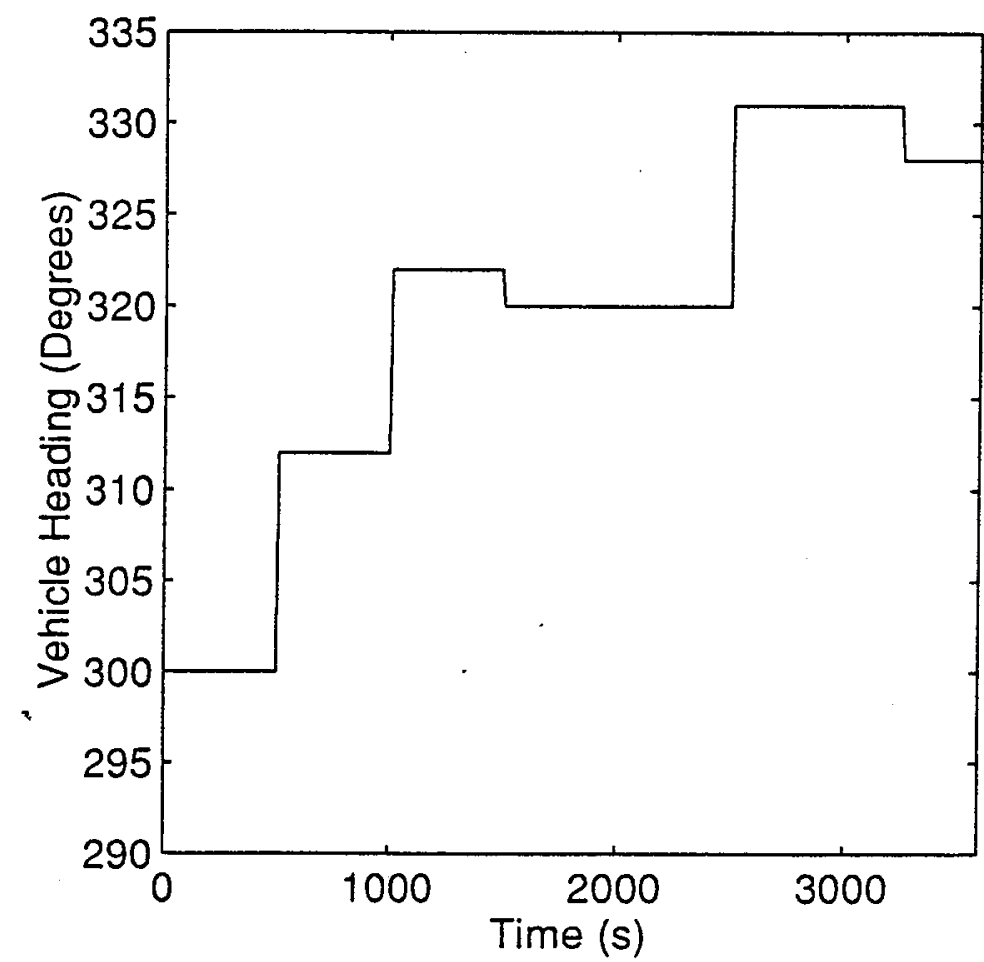

Fig. 4-4. Noise-free measurement of vehicle heading by gyro. 


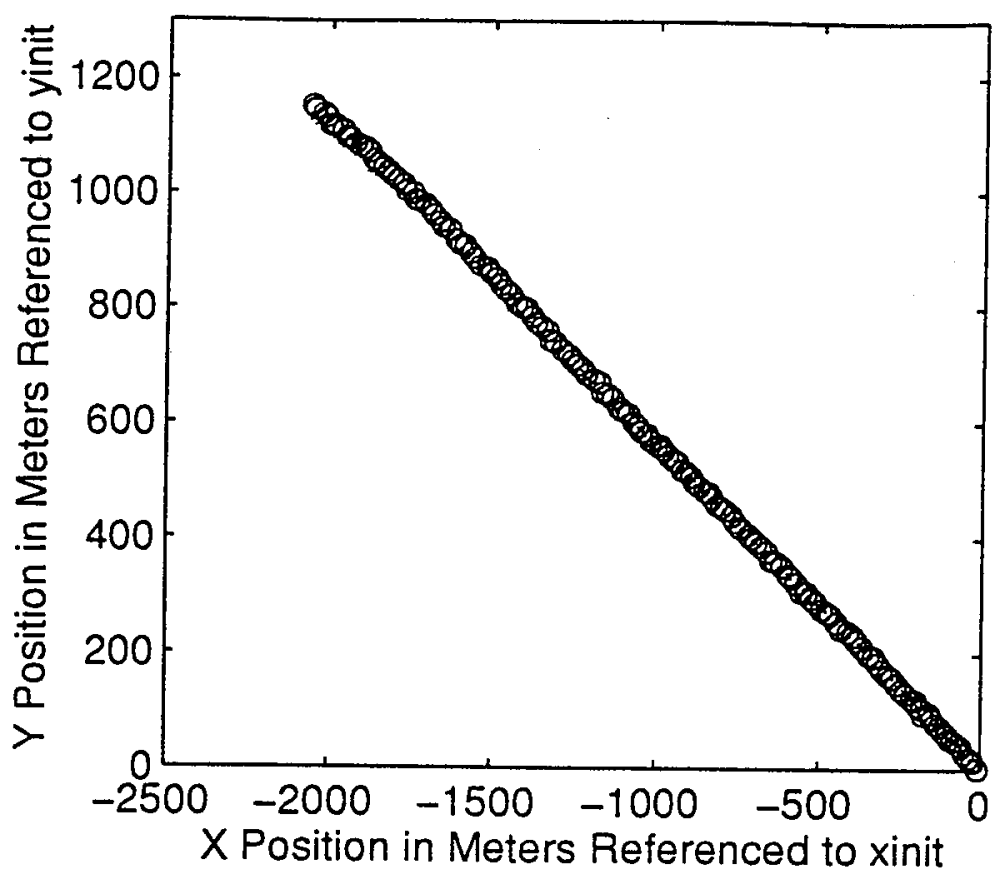

Fig. 4-5. Comparison of measured and estimated X-Y position.

\subsection{Simulation Tests}

\subsubsection{Test 1: Accurate Initial Conditions}

For the first test, doppler velocimeter readings are provided every $10 \mathrm{~s}$ and $\mathrm{X}-\mathrm{Y}$ position information every $100 \mathrm{~s}$. The initial conditions provided for the state variables match their actual values, including gyro and magnetic compass biases. Additionally, a gyro drift of $5^{\circ} / \mathrm{hr}$ is simulated.

Figure 4-5 shows a comparison of actual to estimated position. Position data are available frequently, allowing the filter to maintain a close match between estimated and actual positions. Figure 4-6 provides a close-up of a smaller part of the data to allow better resolution.

Estimating parameters with frequent data updates is relatively easy for the filter to accomplish. For example, Fig. $4-7$ shows a portion of the data set comparing actual and estimated Z-position; Fig. 4-8 shows the sume for roll. Heading is a more difficult state to estimate due to the inherent biases of the available instruments, the gyro and the compass. Figure 4-9 shows the estimate of heading throughout the data run; Fig. 4-10 shows a 


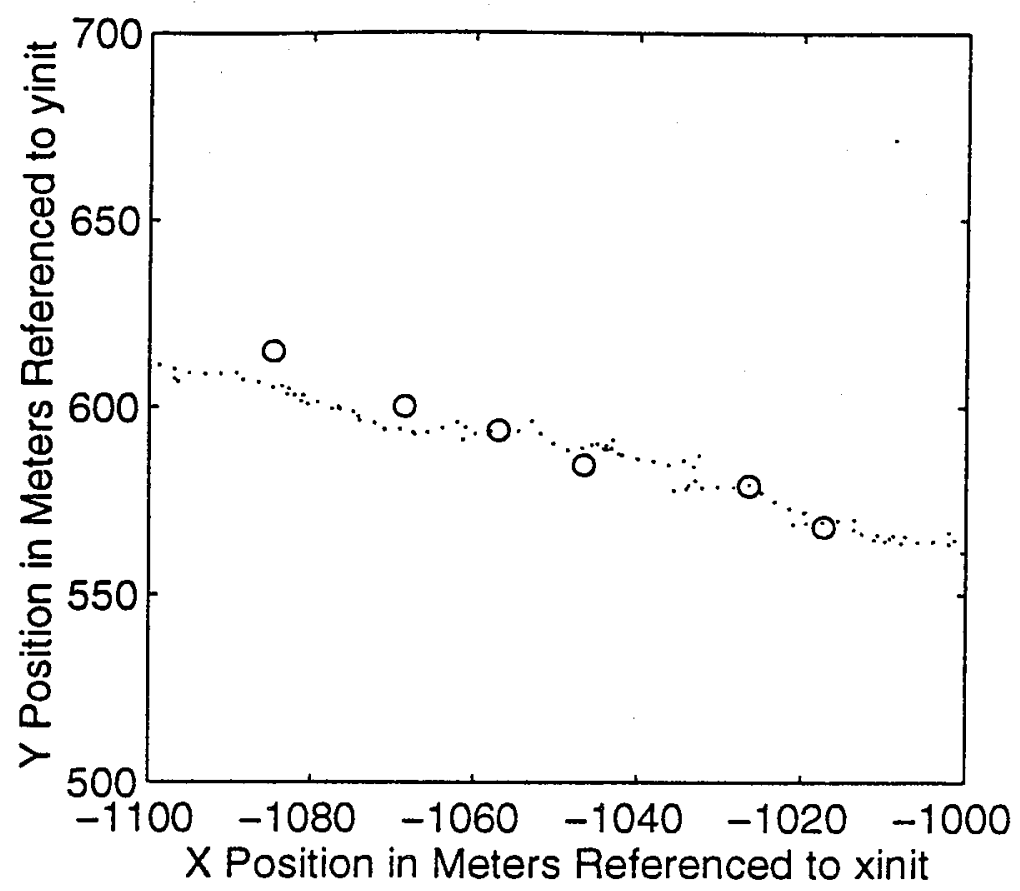

Fig. 4-6. Close-up of X-Y Position Comparison.

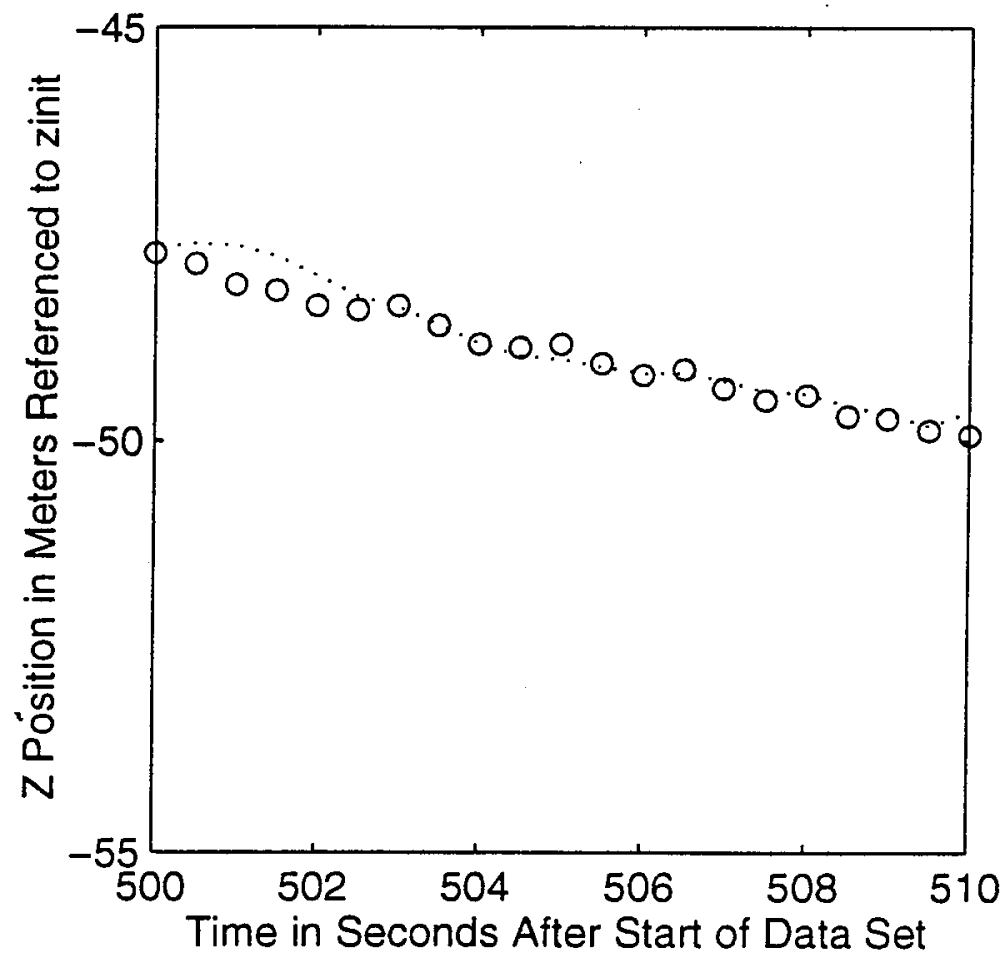

Fig. 4-7. Comparison of actual and estimated Z-position. 


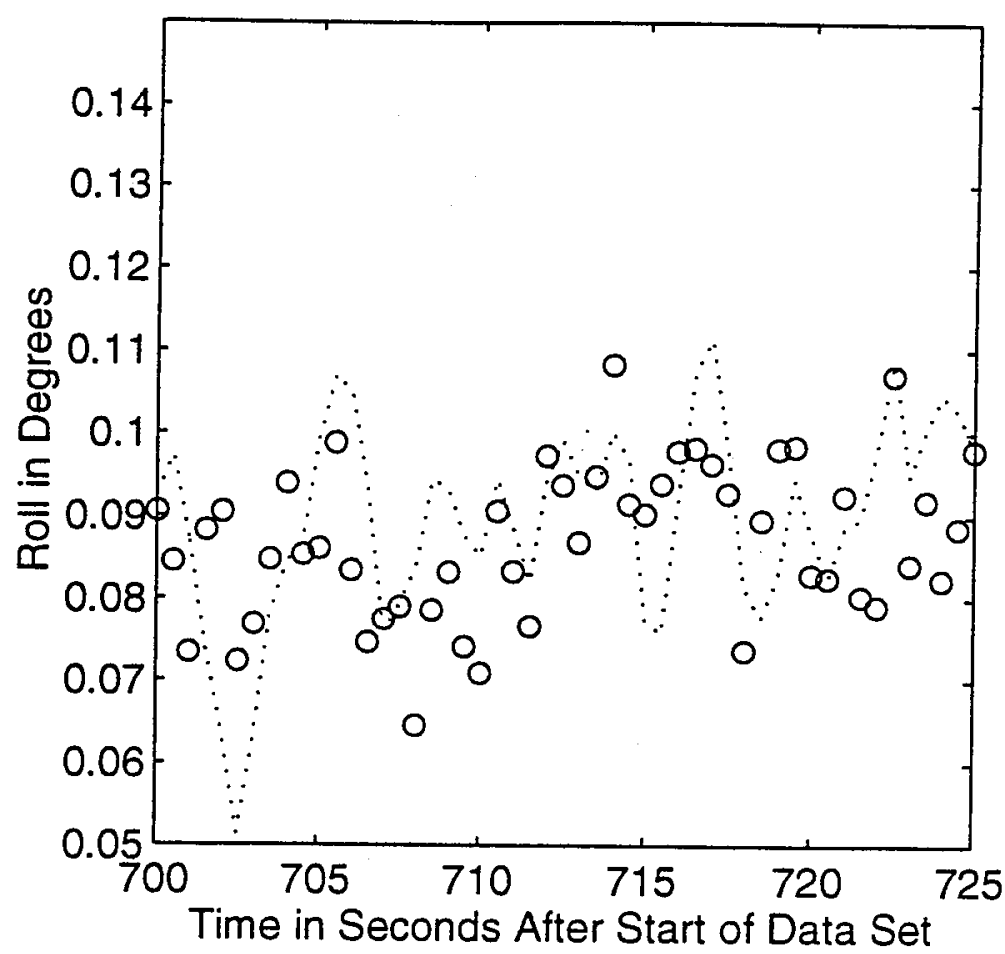

Fig. 4-8. Comparison of actual and estimated roll.

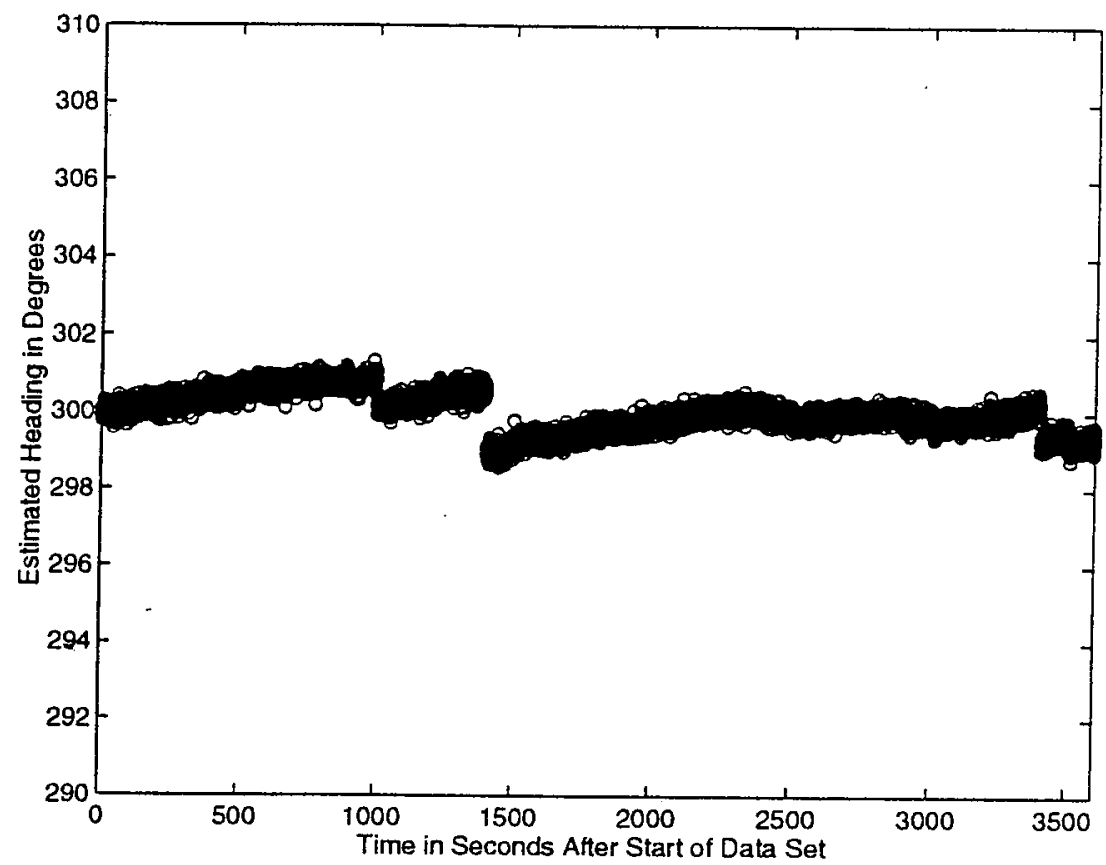

Fig. 4-9. Comparison of actual and estimated heading. 


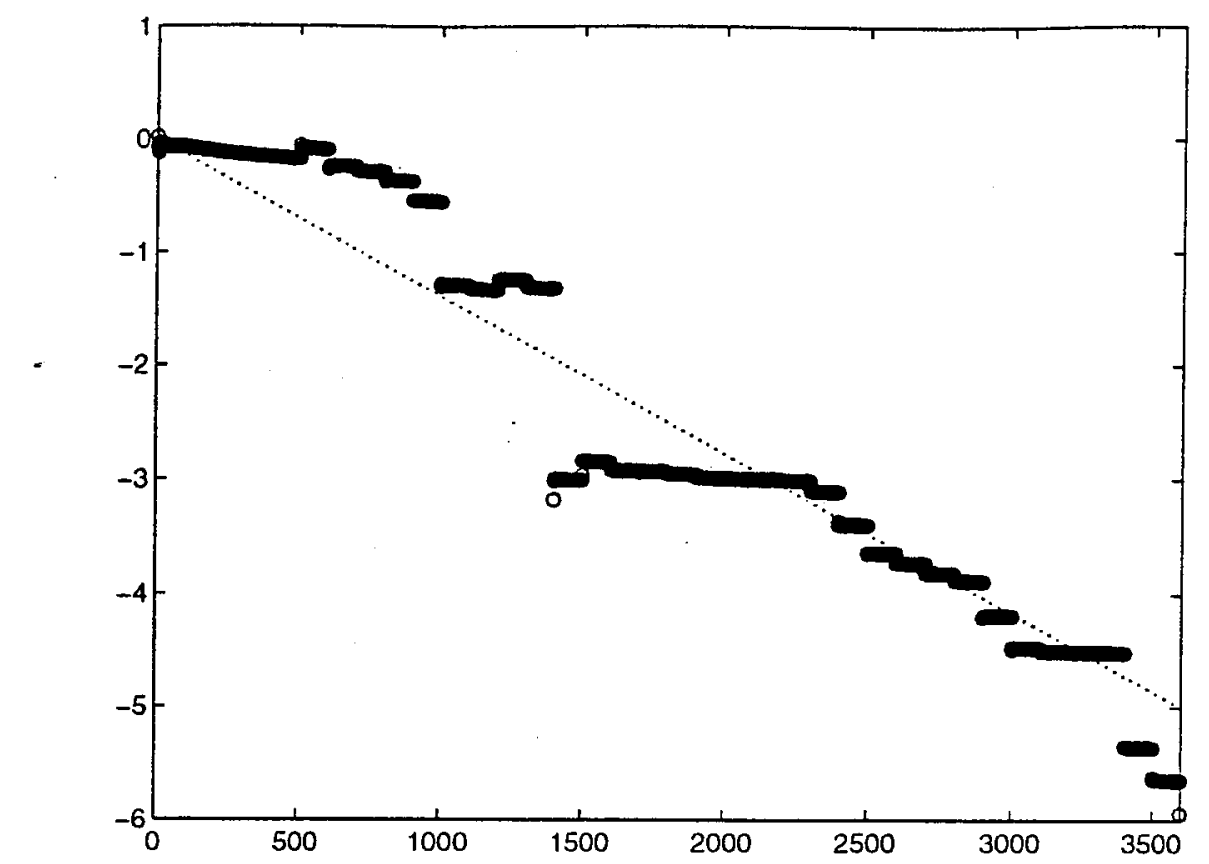

Fig. 4-10. Actual and estimated gyro bias.

comparison of actual and estimated biases for the gyro. With the frequent availability of data, the filter provides a good estimate of the heading and associated biases. Overall, with Gaussian noise and frequent data the filter performs well. Subsequent tests check its performance under more strenuous conditions.

\subsubsection{Test 2: Inaccurate Initial Conditions Combined with Position Data Gap}

For the second test, all parameters are the same except for those noted in Table 4-2. Additionally, no position information is available for a 50)-s interval towards the start of the run..

Table 4.2: Initial Conditions for Simulation Test 2.

\begin{tabular}{|c|c|c|}
\hline Paramieter & Initial Estimate & Actual Value \\
\hline \hline Compass Bias & $-24^{\circ}$ & $-18^{\circ}$ \\
\hline Gyro Bias & $-7^{\circ}$ & $00^{\circ}$ \\
\hline Heading & $307^{\circ}$ & $3000^{\circ}$ \\
\hline
\end{tabular}




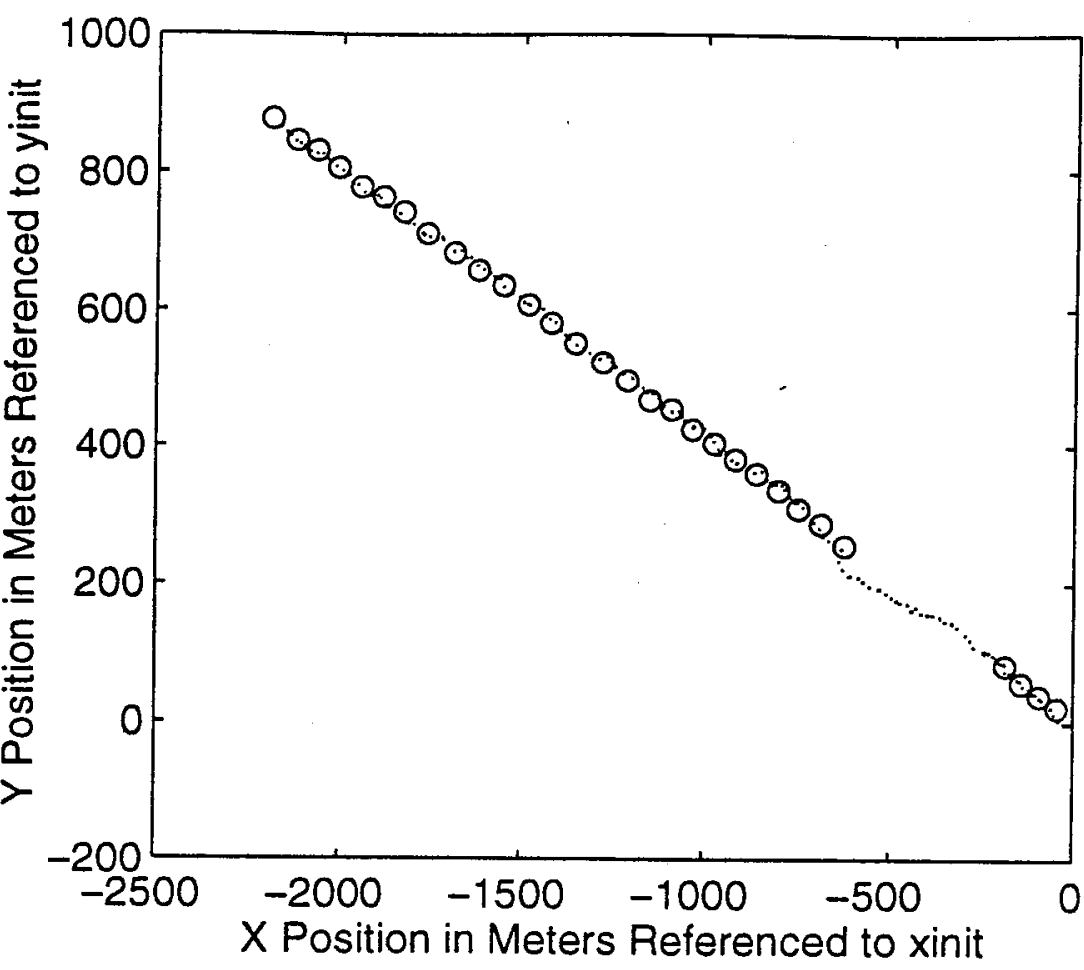

Fig. 4-11. Comparison of measured and estimated $\mathrm{X}-\mathrm{Y}$ positions.

As can be seen in Fig. 4-11, the lack of early position error combined with the incorrect initial heading and hiases causes some inaccuracies during the time without position data. However, after position information is again received the filter quickly adjusts to correct itself.

The large error in the initial heading estimate is somewhat artificial since in actual operation, the towed vehicle should normally be within a few degrees of the surface ship's average heading. However, even with a large initial error heading, gyro bias, and compass bias all approach their actual values by the end of the hour, as seen in Figs. 4-12 through 4-14. If position information had been available throughout the data run, the final estimates would have been even closer.

\subsubsection{Test 3: Estimation Without Doppler Velocimeter}

The last test uses exactly the same parameters as test 2, except that no doppler velocimeter information is included. Under these conditions, an estimate of the importance of the velocimeter can be made. The same graphs are produced for 


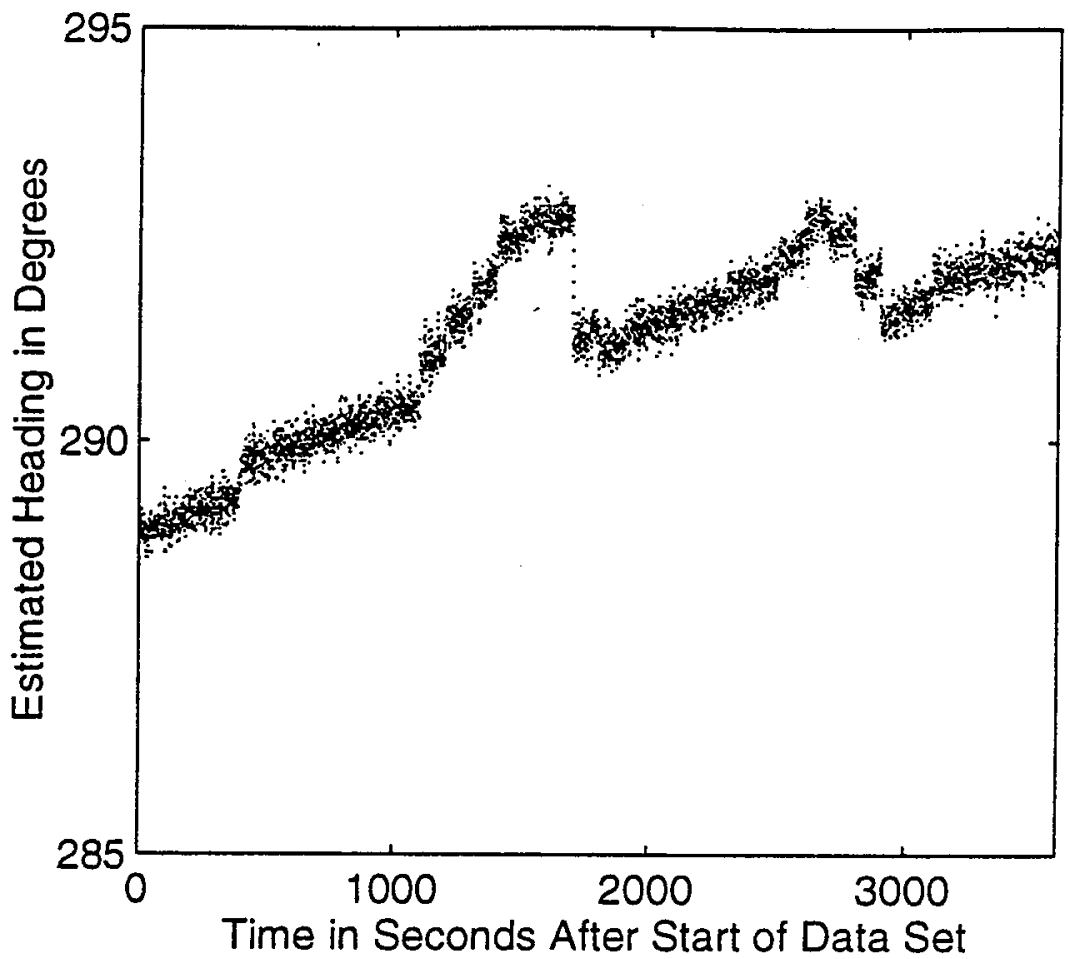

Fig. 4-12. Heading estimate.

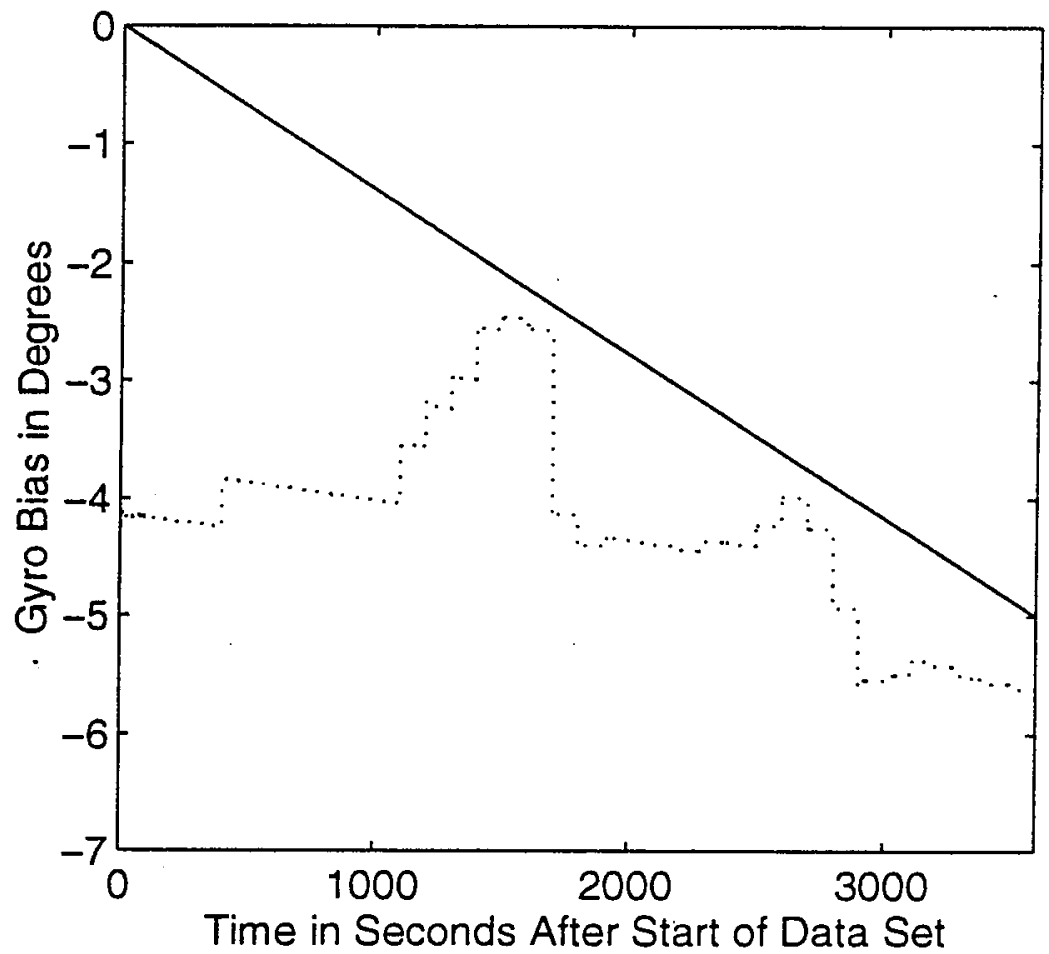

Fig. 4-13. Estimated and actual gyro bias. 


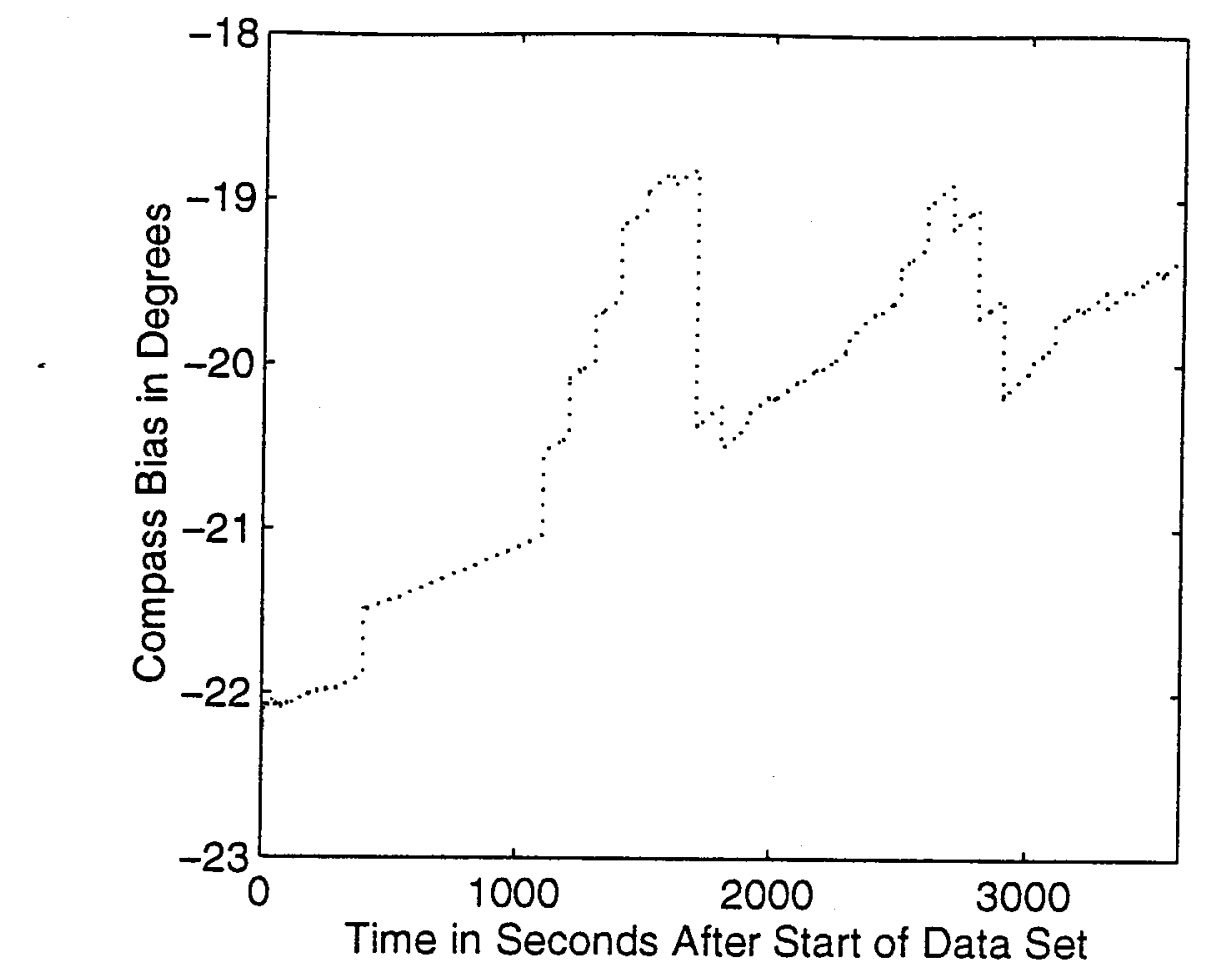

Fig. 4-14. Estimate of magnetic compass bias.

comparison. As can be seen in Figure 4-15, the gup in position data combined with the lack of accurate velocimeter information creates such a large error in position due to velocity errors that all subsequent fixes are rejected by the filter algorithm. This renders position unobservable, and filter divergence results. However, since the compass and gyro are still producing independent sources of heading information, the filter is able to make some corrections to bias. Without fixes to provide true heading measurements, the heading does not converge to its actual value hut uses a weighted average of the gyro and compass measurements to minimize the error. 


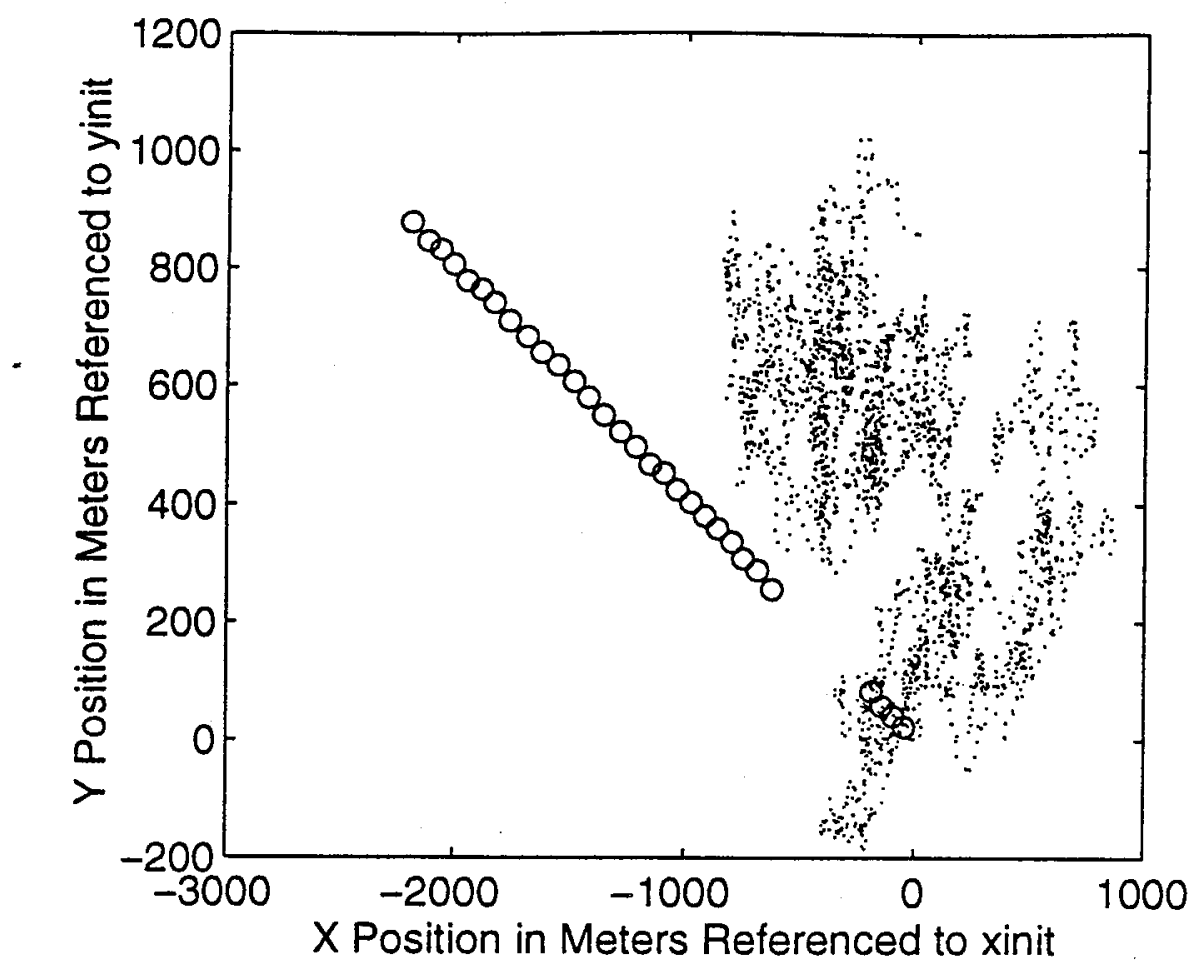

Fig. 4-15. Comparison of measured and estimated X-Y position.

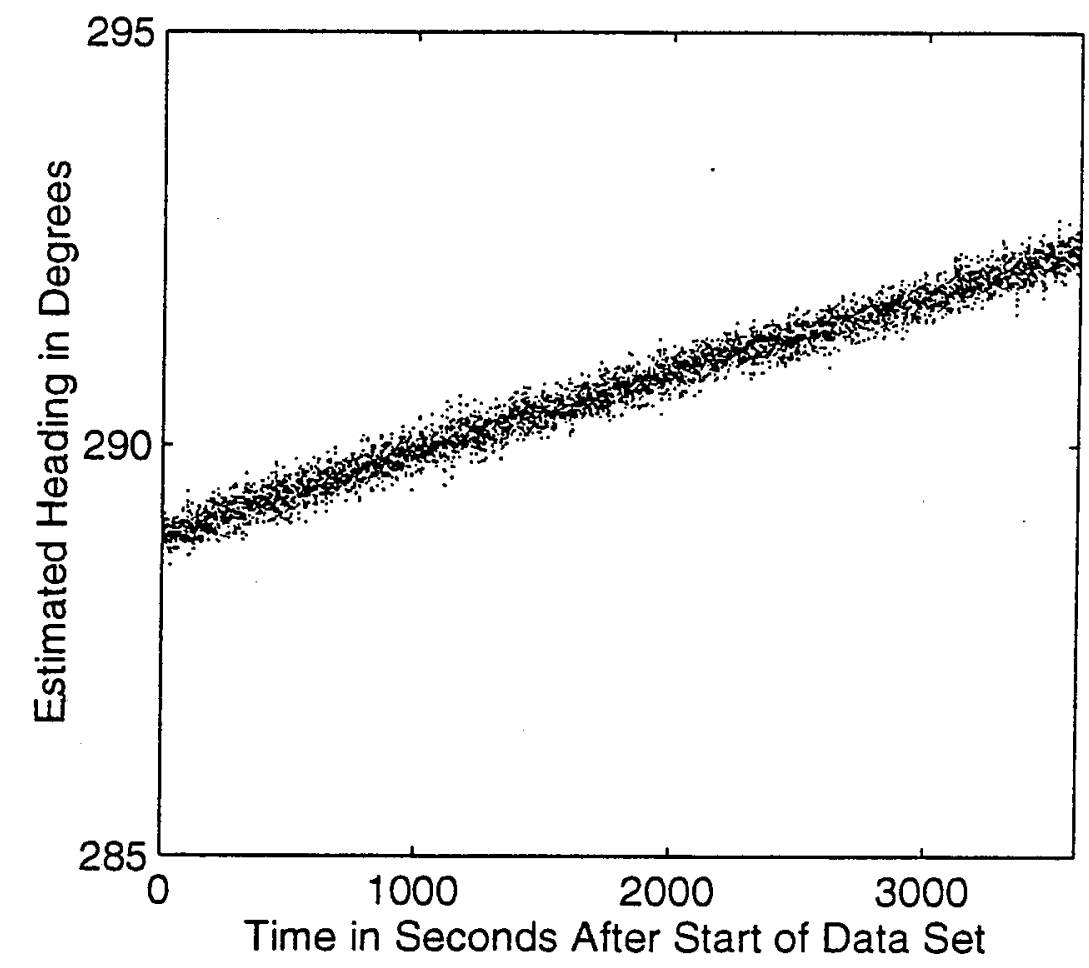

Fig. 4-16. Estimated heading. 


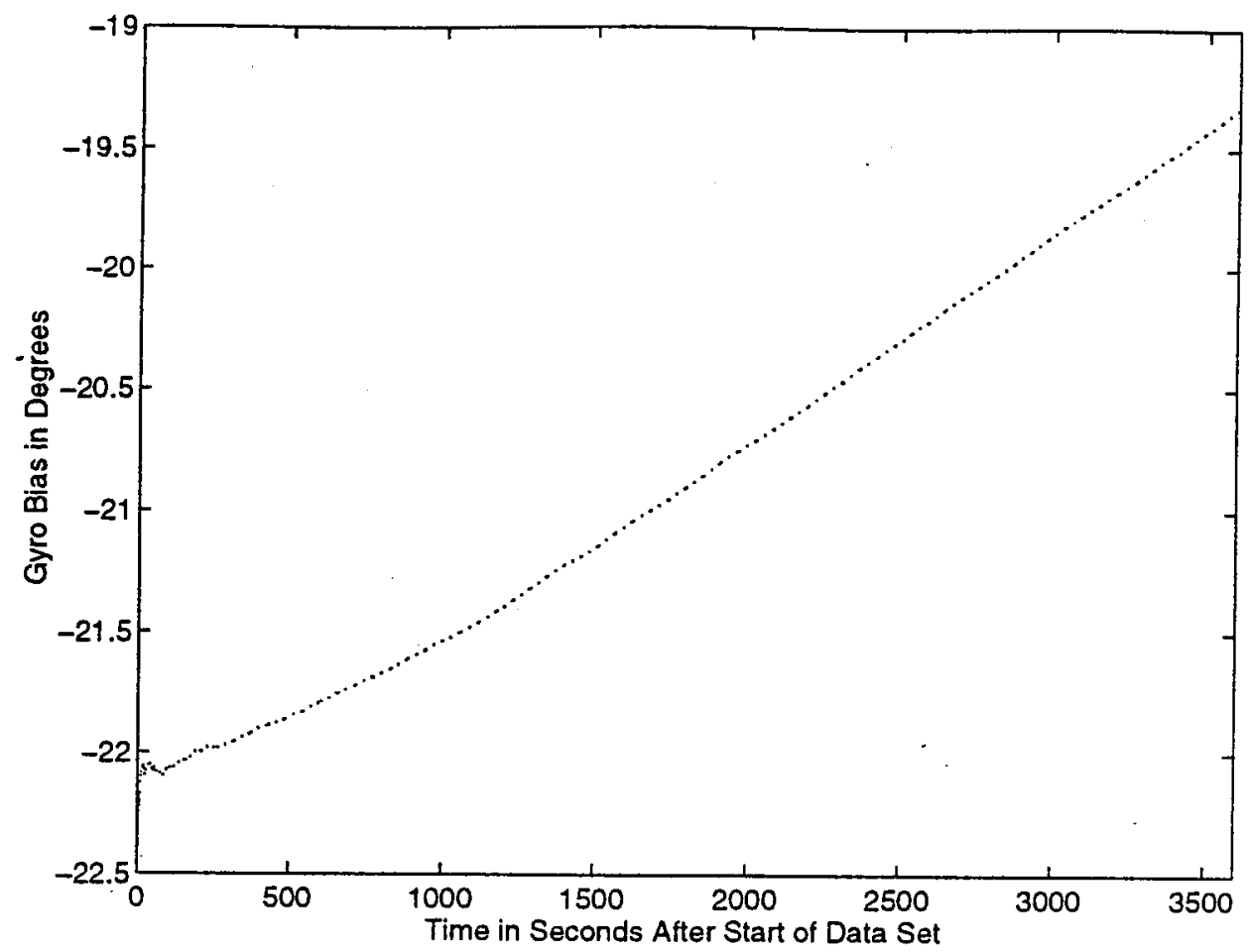

- Fig. 4-17. Estimated magnetic compass bias.

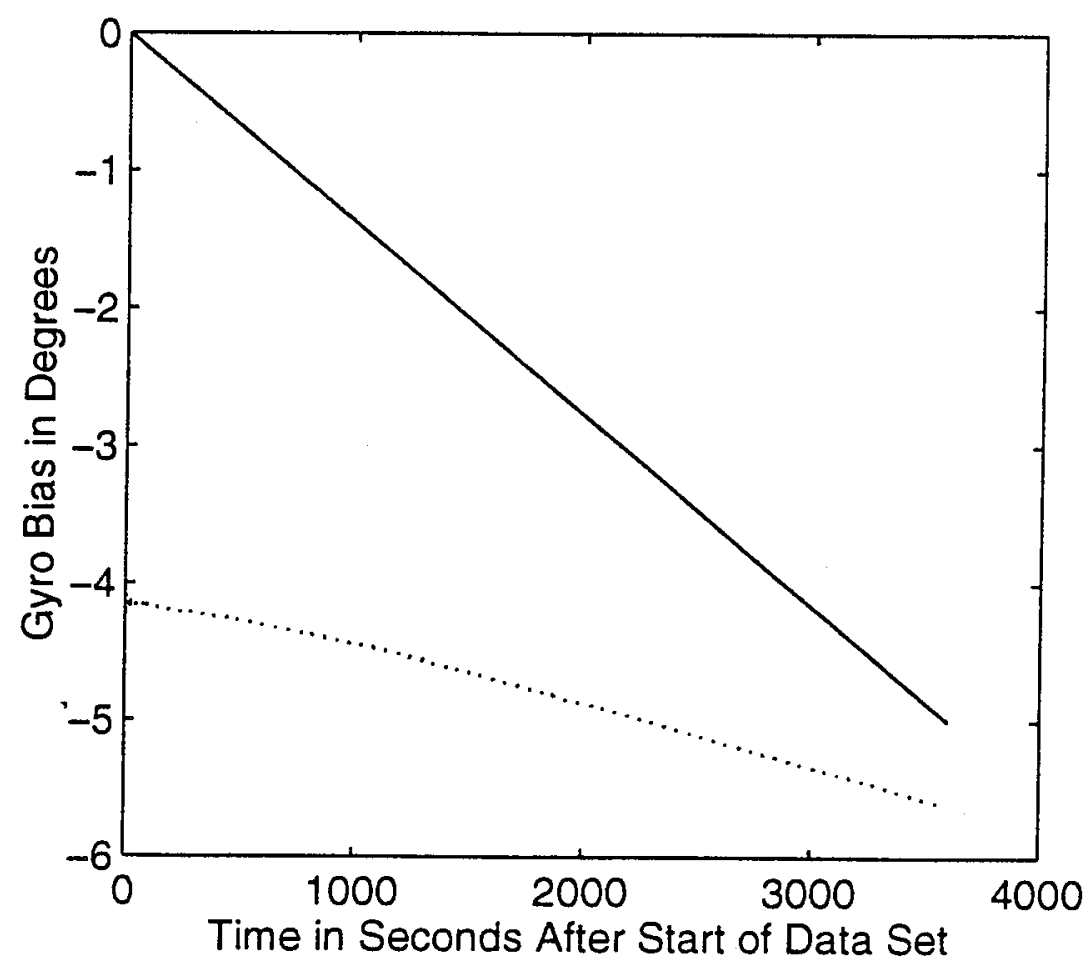

Fig. 4-18. Comparison of actual and estimated gyro bias. 


\section{Chapter 5}

\section{Kalman Filter Performance Using Actual Data}

\subsection{Description of Data}

The data used for this evaluation were obtained in July, 1994, during operation of the DSL-120, a towed vehicle mounting a sidescan sonar. Both data sets used for filter testing comprise approximately one hour of navigational sensor information. Due to the specific characteristics of the data package, two modifications were made to the Kalman filter algorithm to allow proper processing.

First, gyro data were not obtained. Therefore, only the magnetic compass is used as the heading sensor for this data. As a consequence, the order of the filter is reduced by one due to the removal of gyro bias as a state variable.

Second, analysis of the data reveals some anomalies. During the first data run, numerous spurious velocities of approximately $30 \mathrm{~m} / \mathrm{s}$ are observed. In the second data run, regular spurious compass readings of exactly $40^{\circ}$ are found. These anomalies are removed before using the data in the filter.

\subsection{First Data Set}

On initial inspection, the first data set appears to be excellent for Kalman filter use due to the frequency of obtained data, especially from the long-baseline positioning system. However, closer analysis reveals that the behavior of the linear accelerometers is erratic. Figure 5-1 shows an example of this hehavior. The difference between the two concentrations of outputs is $0.08 \mathrm{~g}$, which corresponds to a linear acceleration of approximately $0.8 \mathrm{~m} / \mathrm{s}^{2}$. If the filter uses this erratic accelerometer data, it rapidly diverges due to the large estimated accelerations translating into large, nonexistent velocities. Therefore, to run the filter on this data set the accelerometer measurements are set to zero 


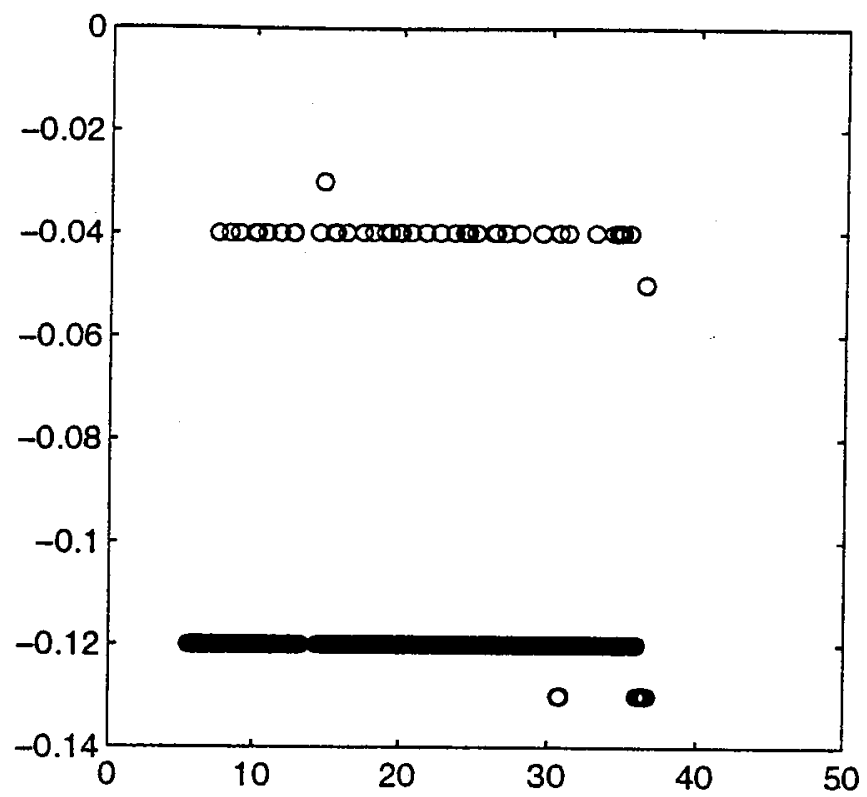

Fig. 5.1. Output of Linear Accelerometer.

for the entire run. The Kalman filter uses the remaining measurements of velocity, position, roll, pitch, and heading to estimate all parameters.

Figures 5-2 and 5-3 show the difference in measured and estimated X-Y positions. The doppler velocimeter provides accurate measurements of velocities, which compensates for the lack of accelerometer measurements. Therefore, the filter is able to track position well.

As an example of a parameter which has the advantage of continuous measurements, a comparison of measured and estimated roll is shown in Fig. 5-4. The discrete character of the sensor measurements compared to the continuous Kalman filter estimate is obvious.

The last estimate meriting special attention is heading. With the frequent fixes, true heading measurements are readily available. As can be seen from Figs. 5-5 and 5-6, occasionally an inaccurate true heading measurement will temporarily affect the estimated heading and bias. 


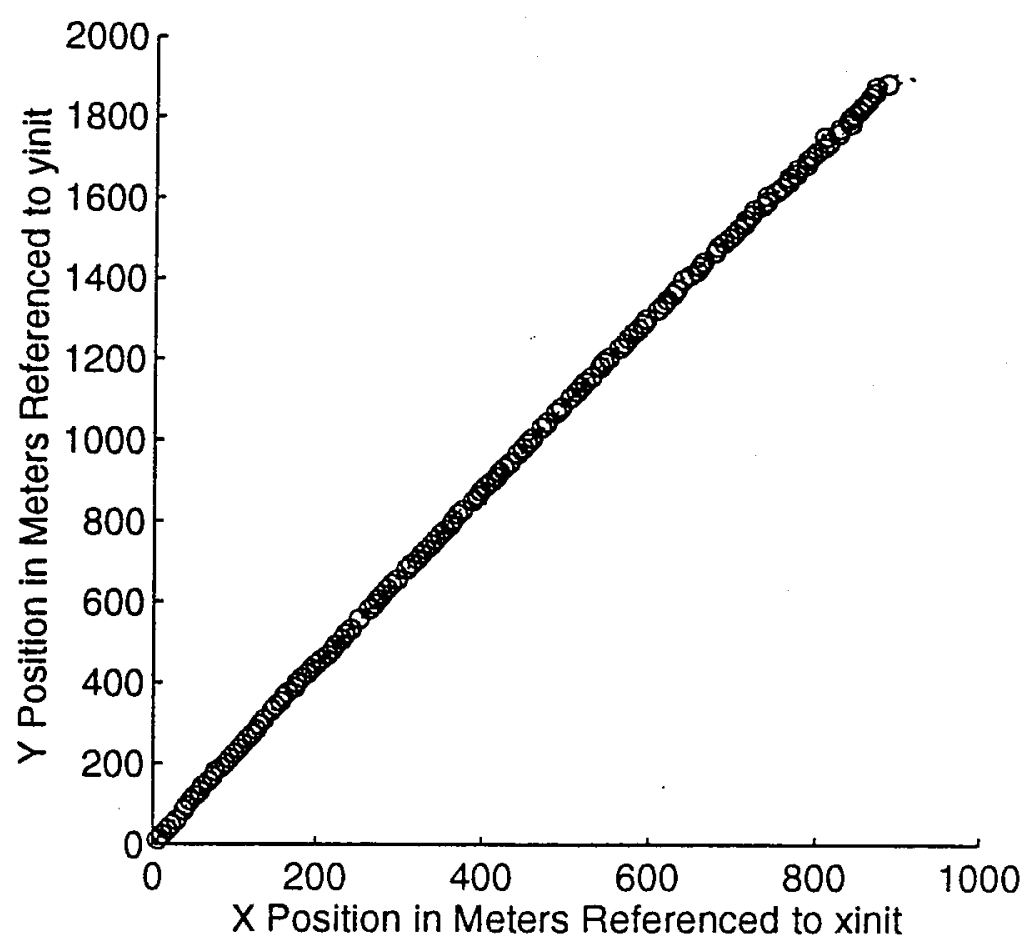

Fig. 5.2. Comparison of estimated and actual X-Y positions.

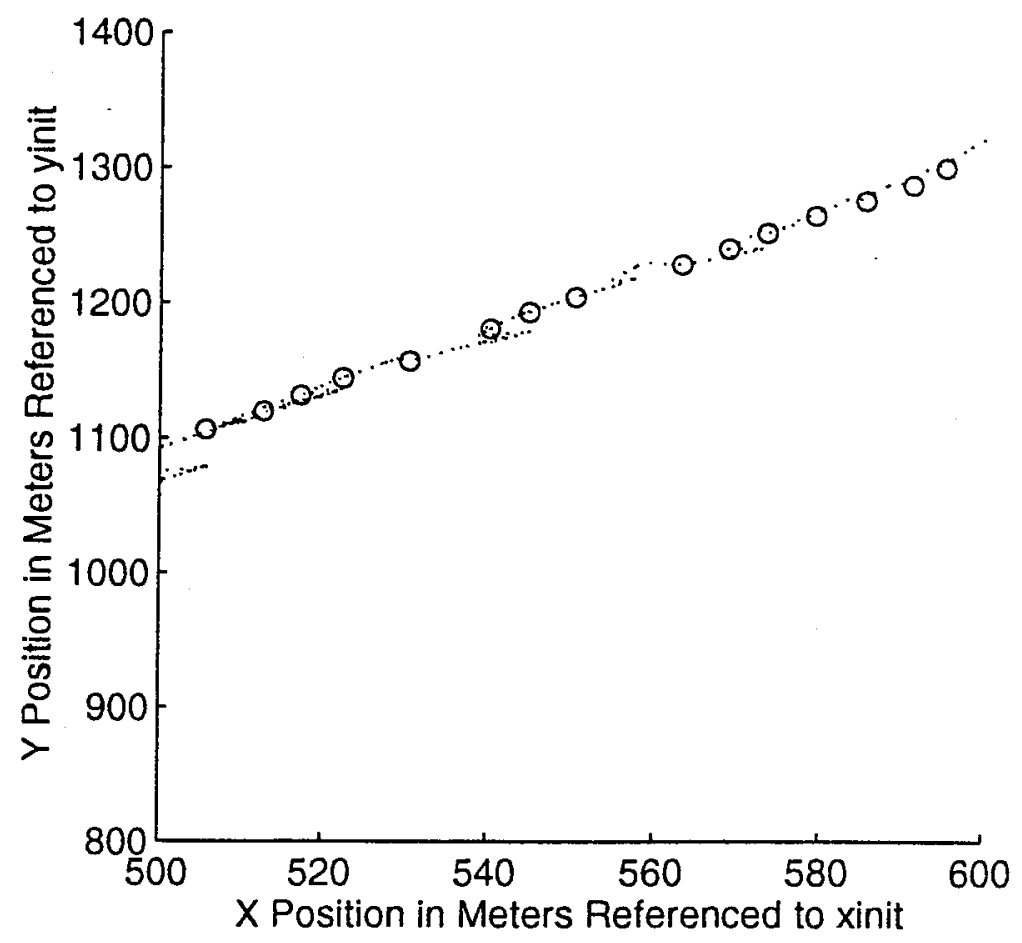

Fig. 5.3. Close-up of $X-Y$ position comparison. 


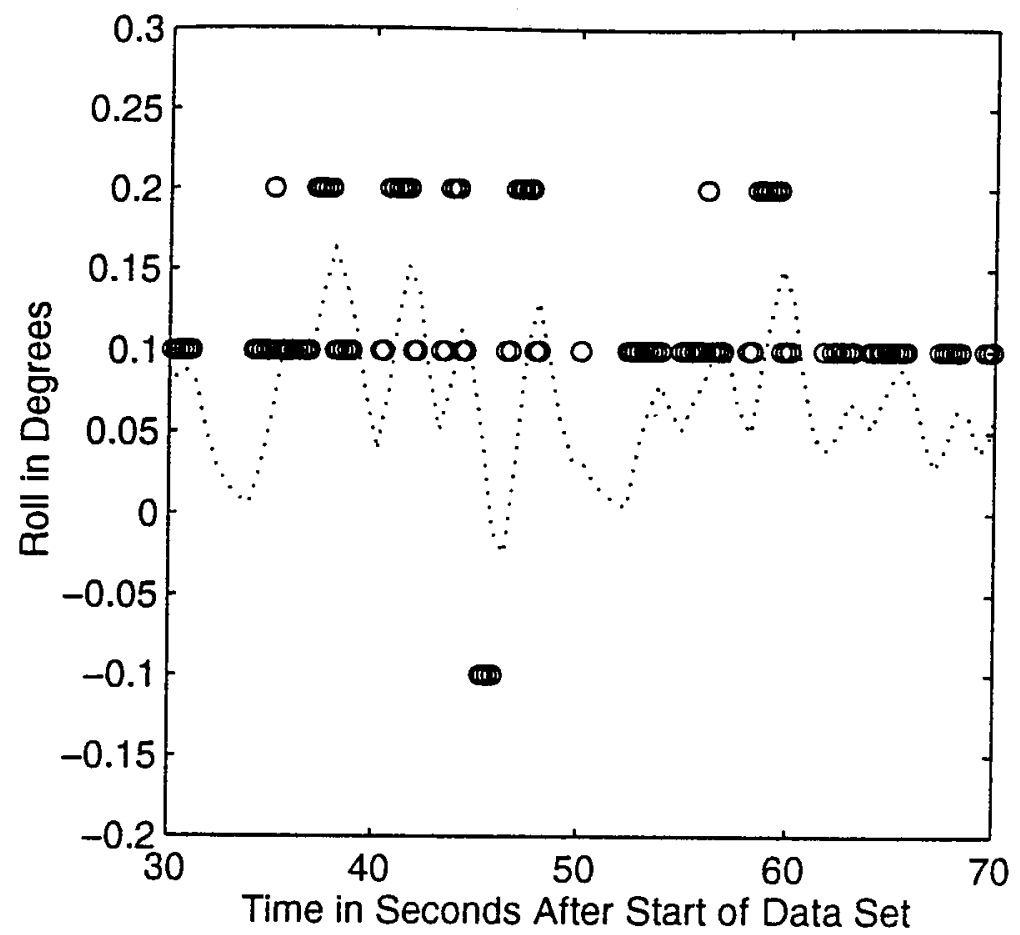

Fig. 5.4. Comparison of measured and estimated roll.

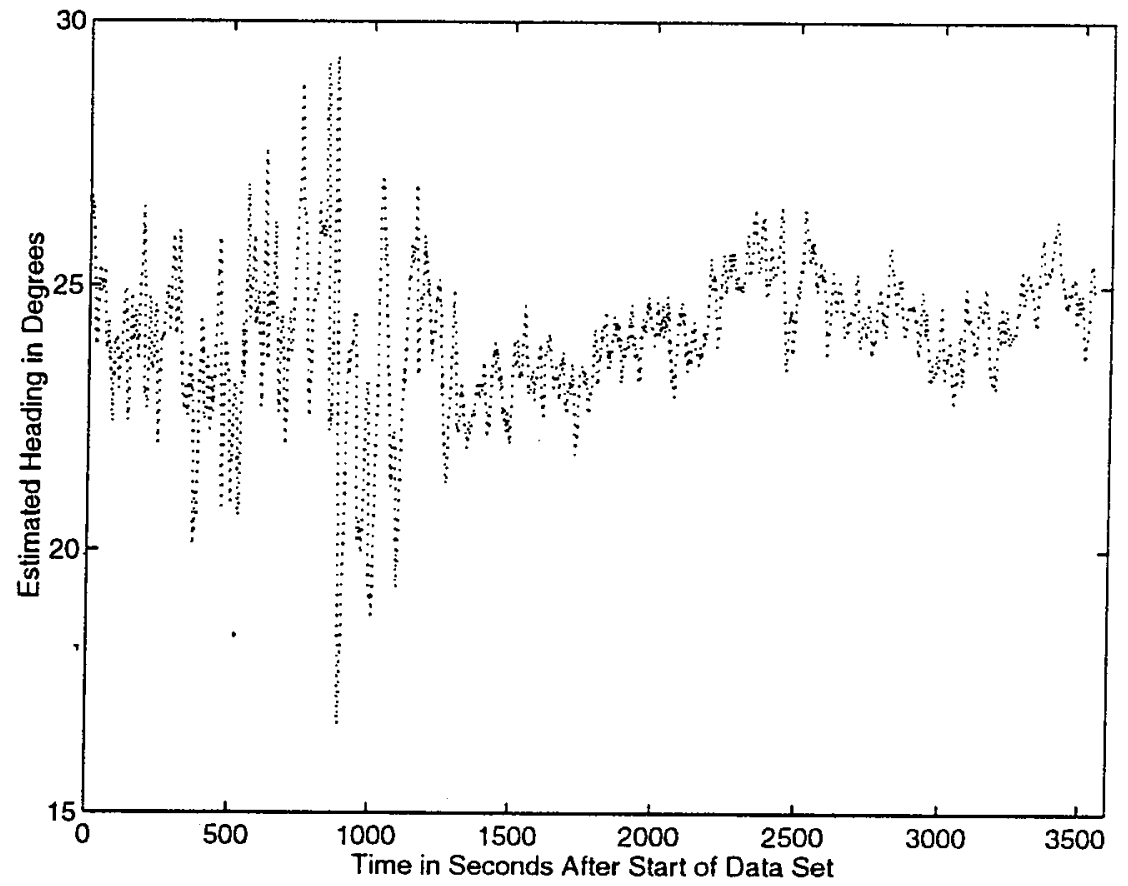

Fig. 5.5. Estimated heading. 


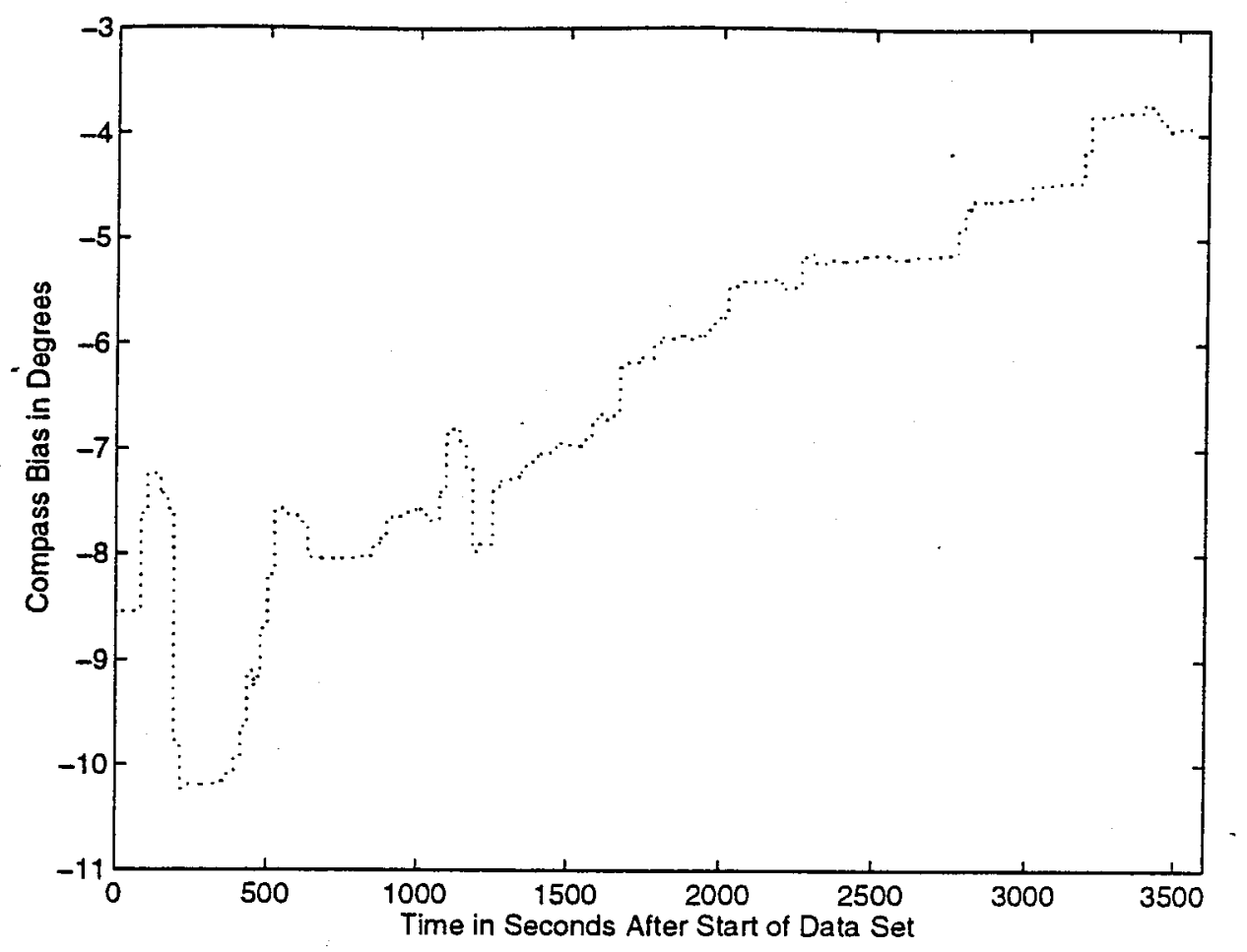

Fig. 5.6. Estimated compass bias.

\subsection{Second Data Set}

The second data set poses more of a challenge for the filter, since after four initial fixes are obtained there is a gap of nearly one-half hour without further XY-position information. However, the linear accelerometers do not show the same erratic behavior during this data set and are therefore used as part of the measurement vector. Figures 5-7 through 5-12 show the performance of the filter in estimating position in light of this data gap. When position information is restored, there is a discontinuity in the filtered estimate of position due to the error developed by the filter estimate during the gap. After frequent fix information is again received, filter position estimates track with actual positions obtained from the long-baseline navigation system.

Besides position, the most important vehicle parameter to estimate accurately is heading. Without an accurate heading, sidescan sonar information will be inaccurate as well. Since both the gyro and the compass can be biased, a vital characteristic of the filter 


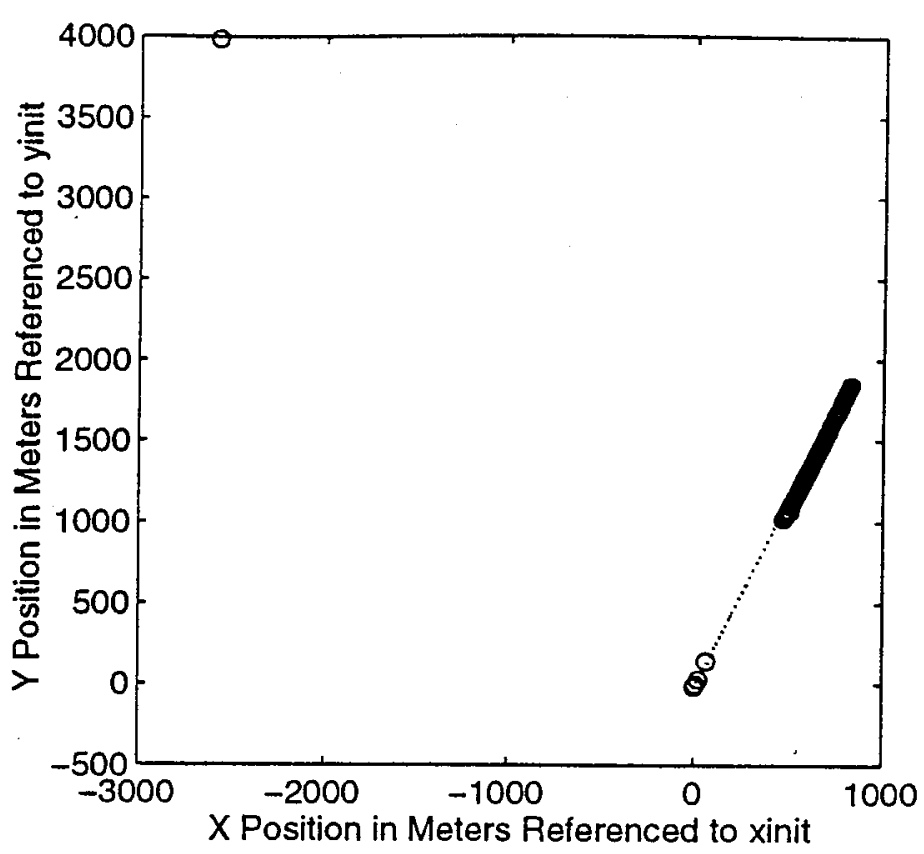

Fig. 5.7. XY-position estimates (:) compared to position fixes (o). Note the presence of a flyer in the upper left corner, which was properly rejected by the filter.

is its ability to estimate heading. Figure $5-112$ shows the estimated heading during the hour. To understand filter behavior in the vicinity of this discontinuity, X-position behavior is examined individually in Fig. 5-10. After a fix is received following the long data gap, the first fix is weighted heavily and the estimated X-position jumps to the location of the fix. As can be seen in the figure, however, that first fix was rather inaccurate, although still within the specification of the fix rejection filter. Subsequent fixes are weighted less heavily since the error covariance matrix is adjusted after receipt of the first fix. Therefore, estimated position adjusts to actual position over several fixes.

The jump in estimated heading at $t=200(0)$ seconds is a result of the gap in position information. However, despite this lengthy gap, the estimated heading remained consistent within three degrees.

Parameters with more frequent data update rates are easier for the filter to estimate since measurements are continuously matched against the estimates. As one example, the comparison of estimated Z-position for a small portion of the hour is shown in Figure 512. 


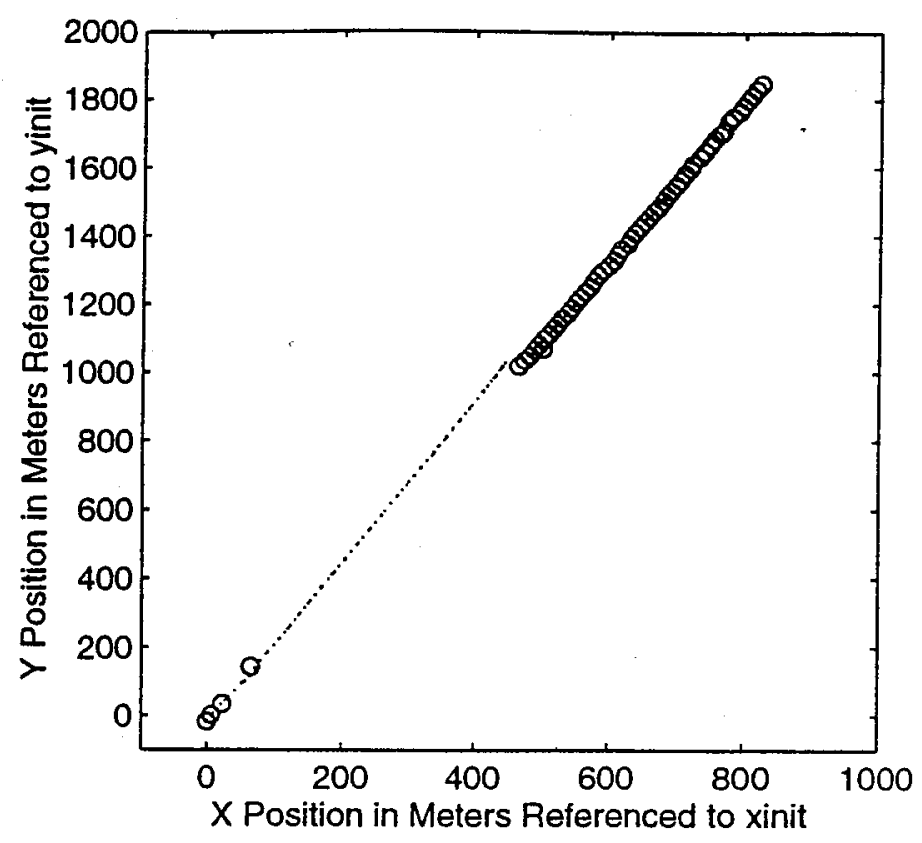

Fig. 5.8. Comparison of estimated and actual vehicle track (blowup of Figure 5-7 with the region of the flyer excluded).

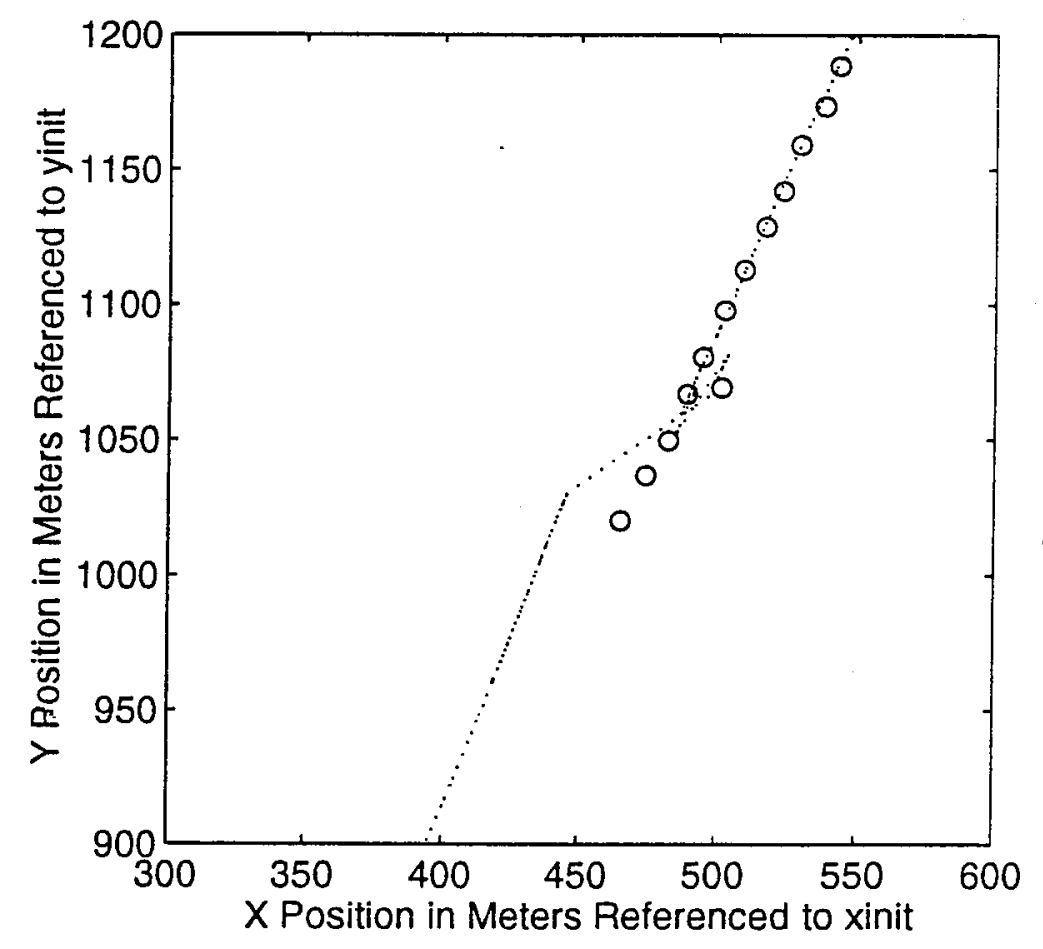

Fig. 5.9. Close-up of region of discontinuity following gap in position information. 


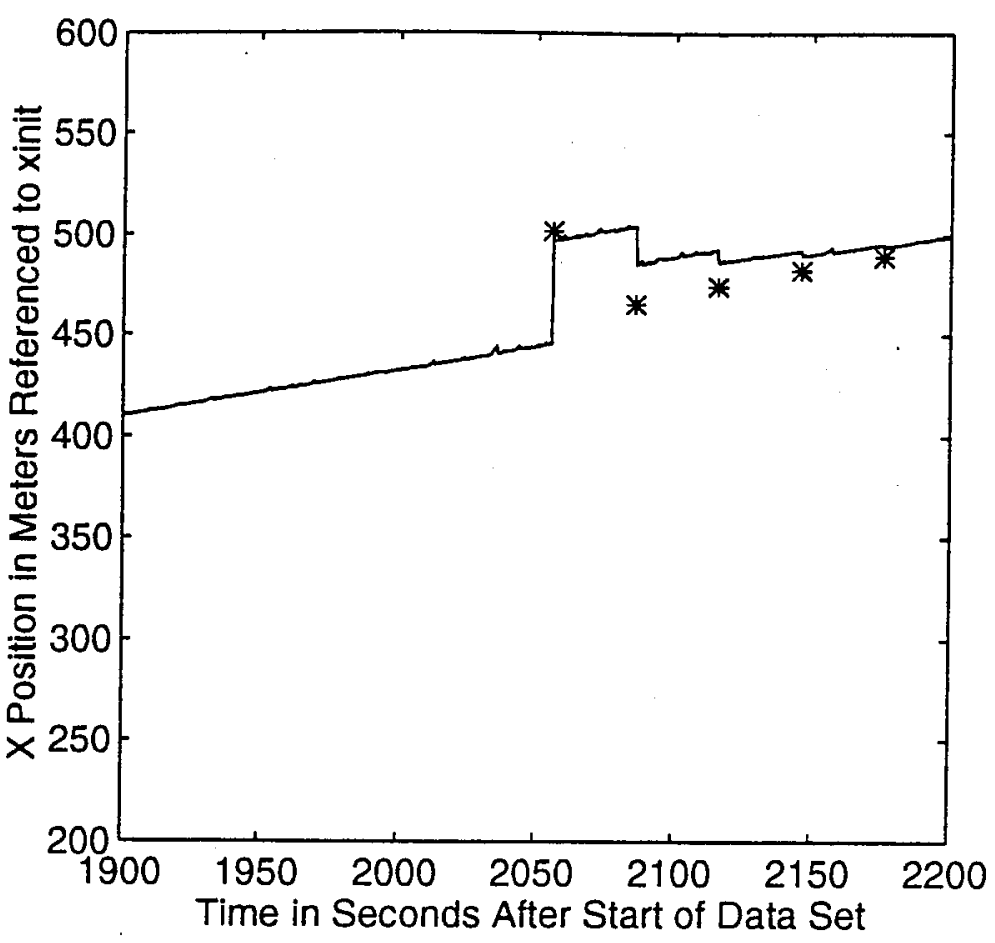

Fig. 5.10. X-position behavior in the vicinity of the discontinuity.

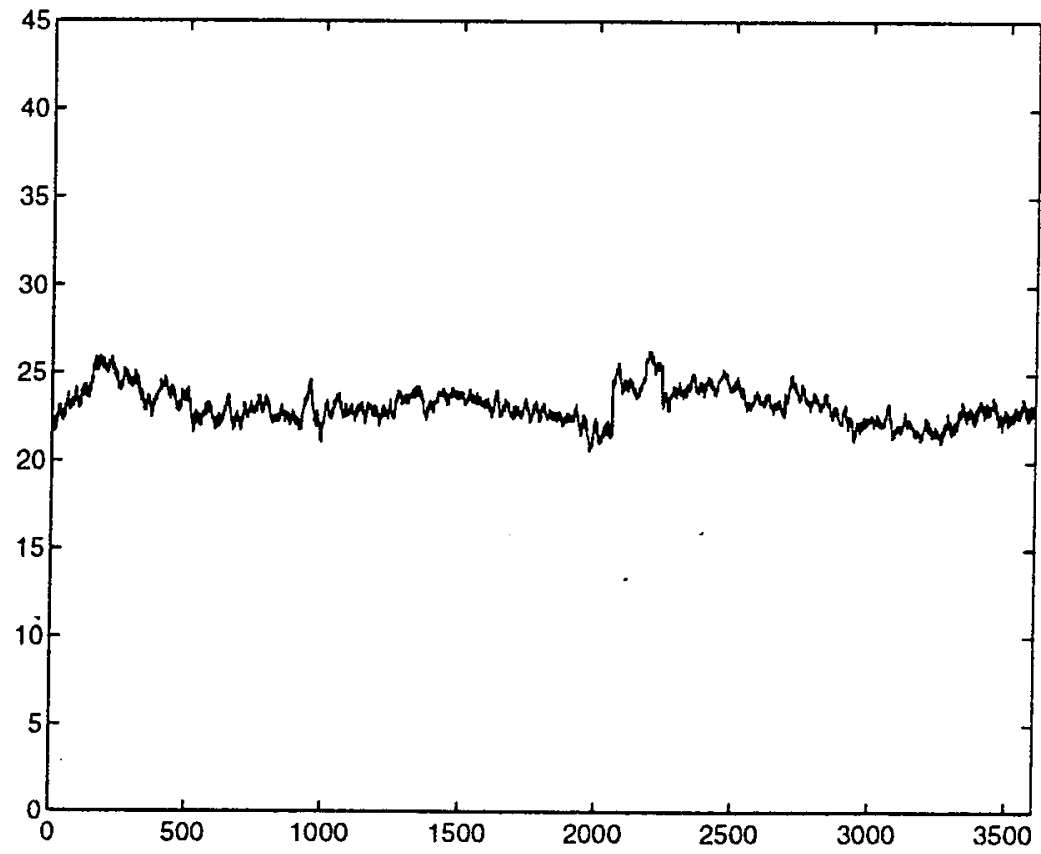

Fig. 5.11. Estimated Heading. 


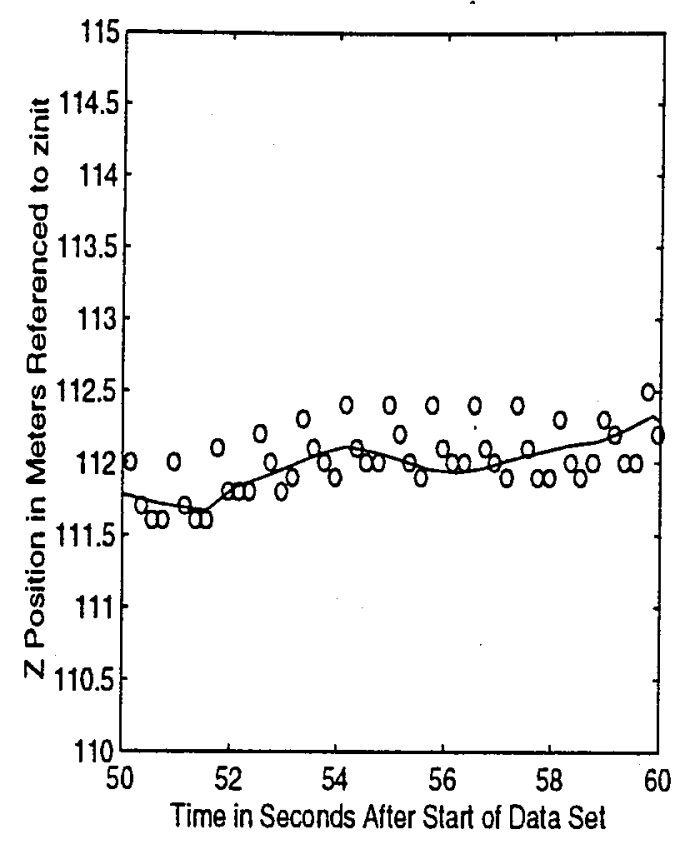

Fig. 5.12. Comparison of measured and estimated Z-position.

With the aid of the doppler velocimeter, the Kalman filter is able to maintain a valid estimate of vehicle parameters during this lengthy gap in position information. The key requirements in achieving this are:

1. The doppler velocimeter is available to provide accurate velocity information throughout the data gap.

2. Prior to losing position information, heading accuracy was sufficient to allow the Kalman filter to bridge the gap while still remaining within the window for fix acceptance once position data is regained. 


\section{Chapter 6}

\section{Conclusions}

\subsection{Fulfillment of Objectives}

The objective of this thesis was to develop an extended Kalman filter that could provide accurate estimates of underwater vehicle position and attitude in real-time. Based on the tests of the filter using both simulated and actual data, I believe that the result is a qualified success. On the positive side, the Kalman filter was able to process the data fast enough to permit real-time processing onboard ship, which would allow major reductions in post-cruise processing costs. Additionally, the real-time processing allows those conducting sidescan surveys to verify that the desired areas have been mapped by checking the navigational estimates.

A second success is that when provided with sufficiently accurate and frequent data, the Kalman filter can provide accurate estimates of all vehicle motion and attitude changes. Even when gaps occur in the positioning system, the accuracy of the doppler velocimeter combined with a good prior estimate of heading allows the filter to maintain sufficient accuracy to arrive close to measured position when position data is regained. Also, the Kalman filter has the ability to estimate gyro bias and compass bias to compensate for inaccurate initial heading information and the effects of gyro drift. Therefore, the heading estimate becomes increasingly accurate as fix information is received and the filter is allowed to update over longer periods of time.

In contrast to the successes, there were also some failures. Many of these can be attributed to sensor limitations, especially those of the linear accelerometers contained within the IMU package. A constant bias can be compensated for, but in the first datid run there was an example of erratic accelerometer behavior that threatened the viability of the entire estimation process. Only by recognizing this problem and relying on the doppler velocimeter entirely could accurate estimates of vehicle position be maintained. While in principle the Kalman filter is designed to permit any measurement to be used despite its noise characteristics, this application tests that theory due to the varying data rates of the 
sensors. For a "standard" Kalman filter, all measurements are obtained at every filter update step. Therefore, noisy measurements can be made nearly meaningless by assigning greater inherent accuracy to less noisy ones. In this case, that is not possible. When the only available measurement in the series of integrations leading to position is acceleration, the filter is forced to use it. Even though its weight is initially low, over time these measurements have a significant effect on the estimates of velocity and position until other sensor data are received.

A second concern is whether the heading estimates provided by the filter are accurate enough to be truly useful for sidescan sonar mapping operation. When scanning at long range, even a few tenths of a degree of heading error can be significant. While heading accuracy should continue to improve as the filter operates for longer periods, a compromise is always necessary when deciding how to weight old measurements. If old measurements are weighted too heavily, the filter does not respond to actual changes in state variables, such as a change in course. On the other hand, weighting old measurements less results in greater filter movement toward new measurements with subsequent instability and potentially erroneous changes in bias estimates.

Finally, the filter as presently constructed is of limited utility in estimating vehicle motion for a maneuvering vehicle. While, theoretically, control inputs could be added fairly easily, the modeling necessary for accurate results is not yet a reality. While most current operations involving a thruster-controlled vehicle are limited to a small area and can therefore take advantage of a high update rate for position information, future advances in vehicle technology promise a long-range, independently-operating autonomous vehicle. As constructed here, it is doubtful that the Kalman filter can provide sufficient accuracy given the sensors available.

\subsection{Future Work}

There is much work that could be done to improve the performance of this Kalman filter estimation process. A few possibilities are as follows.

First and most obviously, improved sensors would translate directly into improved measurements. The primary candidates for such improvements are the linear 
accelerometers and the gyro. Without gyro stabilization, it is difficult to improve accelerometer stability. However, improvements continue to be made, and it is hoped that cost, weight, and power requirements will continue to decline to allow better acceleration measurements. As an adjunct to this effort, more testing needs to be performed on the accelerometers in use to understand their characteristics and limitations more fully. Hopefully, the ring-laser gyro will soon become inexpensive enough for practical use in this application to improve heading measurements. Also, the method of obtaining true heading used here is only one possibility. Any improvements in true heading accuracy would improve the estimates of both heading and heading sensor bias.

To allow this filter to be useful for such highly maneuverable vehicles as Jason, further work needs to be carried out on modeling control inputs to the filter. Additionally, if a strapdown IMU package is still in use, the addition of the effects of angular velocities on linear acceleration and velocities may have to be considered due to the greater angular velocities involved.

Finally, there are always refinements which can be made to improve filter performance. One possibility is to add to the state vector estimates for other sensor biases. This has the potential for improving all measurements, especially those of the accelerometers, which appear to have biases that are always present and frequently changing. 


\section{Bibliography}

Britting, K., Inertial Navigation Systems Analysis, Wiley-Interscience, New York, 1971.

Brown, R. and P. Hwang, Introduction to Random Signals and Applied Kalman Filtering. 2nd Edn., John Wiley \& Sons, New York, 1992.

Cady, W., Q-Flex® Accelerometer Construction and Principle of Operation, Technical Note TN-103, Sundstrand Data Control, Inc., Redmond, WA, 1984.

Catford, J., "Application of Strapdown Inertial Systems With Particular Reference to Underwater Vehicles," in NATO Advisory Group for Aerospace Research \& Development (AGARD) Lecture Series No. 95: Strapdown Inertial Systems, Technical Editing and Reproduction, Ltd., London, 1978.

D'Appolito, J., The Evaluation of Kalman Filter Designs for Multisensor Integrated Navigation Systems, Analytic Sciences Corp. Technical Report AFAL-TR-70-27 1, 1971.

Di Massa, D., Operation of an Autonomous Underwater Vehicle Using Hyperbolic Navigation, M.E. Thesis, MIT/WHOI, 1992.

Gelb, A., ed., Applied Optimal Estimation, M.I.T. Press, Cambridge, MA, 1974.

KVH Industries, Inc., C-10) Compass Engine Technical Manual, Rev. D, Middletown, RI, 1992.

Morgan, M., Dynamic Positioning of Offshore Vessels, The Petroleum Publishing Company, Tulsa, 1978.

Paroscientific, Inc., Standard Operating Procedure, Doc. No. 8099, Redmond, WA, 1987.

RD Instrument, Direct-Reading Broadhand Acoustic Doppler Current Profiler Technical Manual, Change 4, San Diego, 1993.

Rowe, F. and B. Brumley, Integrated Navigation System Development, Phase I Final Report, RD Instruments, San Diego, 1992.

Somers, T., "Precise Tracking of Underwater Sensors," in Proceedings of the IEEE Oceans '91 Conference, vol.1 pp. 274-279, 1991.

Sorenson, H. and J. Sacks, "Recursive Fading Memory Filtering," in Kalman Filtering: Theory and Application, H. Sorenson, ed., pp. 185-194 IEEE, New York, 1971.

Strang, G., Introduction to Applied Mathematics, Wellesley-Cambridge Press, Cambridge, MA, 1986.

Systron Donner Inertial Division, GyroChip ${ }^{\mathrm{TM}}$ Theory of Operation, Concord, CA, 1993. 
Tracey, B., 1992. Design and Testing of an Acoustic Ultra-short Baseline Navigation System, S.M Thesis, MIT/WHOI, 1992.

VanBronkhorst, A., "Strapdown System Algorithms," in NATO Advisory Group for Aerospace Research \& Development (AGARD) Lecture Series No. 95: Strapdown Inertial Systems, Technical Editing and Reproduction, Ltd., London, 1978.

Vaughn, G., Hybrid State Estimators for the Control of Remotely Operated Underwater Vehicles, O.E./S.M. Thesis, MIT/WHOI, 1988.

Watson Industries, Inc., Watson Inclinometer With Angular Rate Owner's Manual, Eau Claire, WI, 199().

Yoerger, D. and D. Mindell, "Precise Navigation and Control of an ROV at 2200 Meters Depth," in Proceedings of the MTS ROV/ntervention '92 Conference, 1992. 


\section{Appendix}

\section{KFSetup Program}

\% This program provides the initial conditions required to run the Kalman

\% filter algorithm.

DTOR $=$ pi $180 ; \%$ Conversion of degrees to radians

\% The A matrix propagates the state variables

$A=$ zeros $(17)$;

$\mathrm{A}(4: 6,1: 3)=$ eye $(3)$;

$A(13: 15,10: 12)=\operatorname{eye}(3)$;

\% The $\mathrm{G}$ matrix provides the effects of inputs (accelerations and angular

$\%$ velocities) on the state variables

$\mathrm{G}=\operatorname{zeros}(17,6)$

$\mathrm{G}(1: 3,1: 3)=\operatorname{eye}(3)$

$\mathrm{G}(10: 12,4: 6)=\operatorname{eye}(3)$;

\% The $\mathrm{C}$ matrix is the measurement matrix

$\mathrm{CHDG}=[1-10 ; 10-1 ; 100] ; \% 3 \times 3$ matrix used to form heading portions of $\mathrm{C}$ matrices

$\mathrm{CF}=$ eye(17);\% Full $\mathrm{C}$ matrix when all into available

$\mathrm{CF}(15: 17,15: 17)=\mathrm{CHDG}$;

$\mathrm{CNV}=\operatorname{zeros}(14,17) ; \% \mathrm{C}$ matrix when no velocity info available

$\operatorname{CNV}(1: 3,1: 3)=\operatorname{eye}(3)$

$\operatorname{CNV}(4: 11,7: 14)=\mathrm{eye}(8)$;

CNV $(12: 14,15: 17)=$ CHDG;

$\mathrm{CNP}=$ zeros $(14,17) ; \% \mathrm{C}$ matrix when no position info available

$\operatorname{CNP}(1: 6,1: 6)=\operatorname{eye}(6)$

CNP(7:12,9:14) = eye $(6)$;

$\operatorname{CNP}(13: 14,15: 17)=\operatorname{CHDG}(1: 2,:)$;

CNPV $=$ zeros $(11,17) ; \%$ C matrix when neither position nor velocity available

$\operatorname{CNPV}(1: 3,1: 3)=$ eye $(3)$;

CNPV $(4: 9,9: 14)=\operatorname{eye}(6)$;

CNPV(10:11,15:17) = CHDG $(1: 2,:)$;

$\%$ The $\mathrm{D}$ matrix is necessary only for generating simulated data

$\mathrm{D}=\mathrm{zeros}(17,6)$;

$\mathrm{DNV}=\operatorname{zeros}(14,6)$;

DNP = zeros $(14,6)$;

DNPV = zeros $(11,6)$;

\% Error Covariance Matrix for random forcing function 
$\mathrm{Q}=\operatorname{zeros}(6)$

$\mathrm{QXYZ}=(0.2 *$ eye $(3)) . \wedge 2 ; \%$ Set variance for accelerometers

$\mathrm{Q}(1: 3,1: 3)=\mathrm{QXYZ}$;

$\mathrm{QRPH}=(2 * \mathrm{DTOR} * \mathrm{eye}(3)) . \wedge 2 ; \%$ Set variance for angular velocimeters

$\mathrm{Q}(4: 6,4: 6)=\mathrm{QRPH}$;

\% Error Covariance Matrix for measurements

$\operatorname{RACC}=\left(0.1^{*}\right.$ eye $\left.(3)\right) .^{\wedge} 2$;

$\mathrm{RAR}=\left(\left(0.5^{*} \mathrm{DTOR}{ }^{*}\right.\right.$ eye $\left.(3)\right) .^{\wedge} 2$;

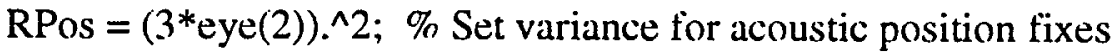

RDepth $=0.1^{\wedge} 2 ; \%$ Set variance for depth

$\mathrm{RPR}=\left(0.5^{*} \mathrm{DTOR}{ }^{*}\right.$ eye $\left.(2)\right) .^{\wedge} 2 ; \%$ Set variance for pitch and roll

$\mathrm{RHG}=\left(0.1^{*} \mathrm{DTOR}\right)^{\wedge} 2 ; \%$ Set variance for heading by gyro

$\mathrm{RHM}=\left(.5^{*} \mathrm{DTOR}\right)^{\wedge} 2 ; \quad \%$ Set variance for heading by compass

\% Reference Points

xinit $=0$;

yinit $=0$;

tinit $=0$;

zinit $=0$;

magvar $=-24 ; \%$ Set initial magnetic compass bias

gyrovar $=-7 ; \%$ Set gyro bias

$\%$ Initial conditions

$x \_O A C C=[(0 ;() ;()] ;$

$\mathrm{x}_{-}(\mathrm{)AR}=[0 ;() ;()]$

$\mathrm{x} \_0 \mathrm{~V}=[0 ; 0.5 ;()] ; \%$ Velocities $(\mathrm{x}, \mathrm{y}, \mathrm{z})$

$\mathrm{x}_{-}(0 \mathrm{XY}=[0$-xinit; $)$-yinit]; \% Position $(\mathrm{x}, \mathrm{y})$

$x_{-}(0 Z=$ zinit -()$; \%$ Position $(z)$

$x_{-}(0 \mathrm{R}=0 * \mathrm{DTOR} ; \%$ Roll

$\mathrm{x}_{-}(\mathrm{PP}=0 * \mathrm{DTOR} ; \%$ Pitch

$\mathrm{x} \_(\mathrm{H}=(30)+\text { gyrovar }) * \mathrm{DTOR} ; \%$ Heading

$\mathrm{x} \_$()

[x_OACC;x_(IV;x_(IXY;x_(OZ;x_()AR;x_0R;x_(OP;x_0H;gyrovar*DTOR;magvar*DTOR] ;

\% Initial Error covariance matrix

P_O = zeros (17); \% Initial P matrix for states

P_o(1:3,1:3) $=(0.1 *$ eye $(3)) .{ }^{\wedge} 2$;

P_o(4:6,4:6) $=(.(1) *$ eye $(3)) .^{\wedge} 2 ; \%$ Initial uncertainty in velocity $(\mathrm{m} / \mathrm{s})$

P_O(7:8,7:8) $=\left(3^{*}\right.$ eye $\left.(2)\right) .^{\wedge} 2 ; \%$ Initial uncertainty in position $(\mathrm{m})$

$P_{-}\left(0(9,9)=0.3^{\wedge} 2 ; \quad \%\right.$ Initial uncertainty in depth $(\mathrm{m})$

P_()(10:12,10:12) $=\left(0.5^{*}\right.$ DTOR *eye $\left.(3)\right) . \wedge 2$;

P_( $(13: 14,13: 14)=\left(1^{*} \mathrm{DTOR} * \text { eye }(2)\right)^{\wedge} 2 ; \%$ Initial uncertainty in roll and pitch

$\mathrm{P}_{-}\left(0(15,15)=(2 * \mathrm{DTOR})^{\wedge} 2 ; \%\right.$ Initial uncertainty in heading

P_o $(16,16)=(2 * D T O R)^{\wedge} 2 ; \%$ Set initial gyro bias variance

$\mathrm{P}_{-}(17,17)=(2 * \mathrm{DTOR})^{\wedge} 2 ; \%$ Set initial magnetic compass hias variance 
$\mathrm{w}=$ zeros $(6,1) ; \%$ random forcing function

$\mathrm{g}=-9.8 ; \%$ gravity vector

\% Set IMU biases to be subtracted from raw data (units of $\mathrm{g}$ for acc, degrees/s for $\%$ angular rates)

xaccbias $=0$;

yacchias $=0$

zaccbias $=0$;

rrbias $=0$;

prbias $=0$

yrbias $=0$; 


\section{KFRun}

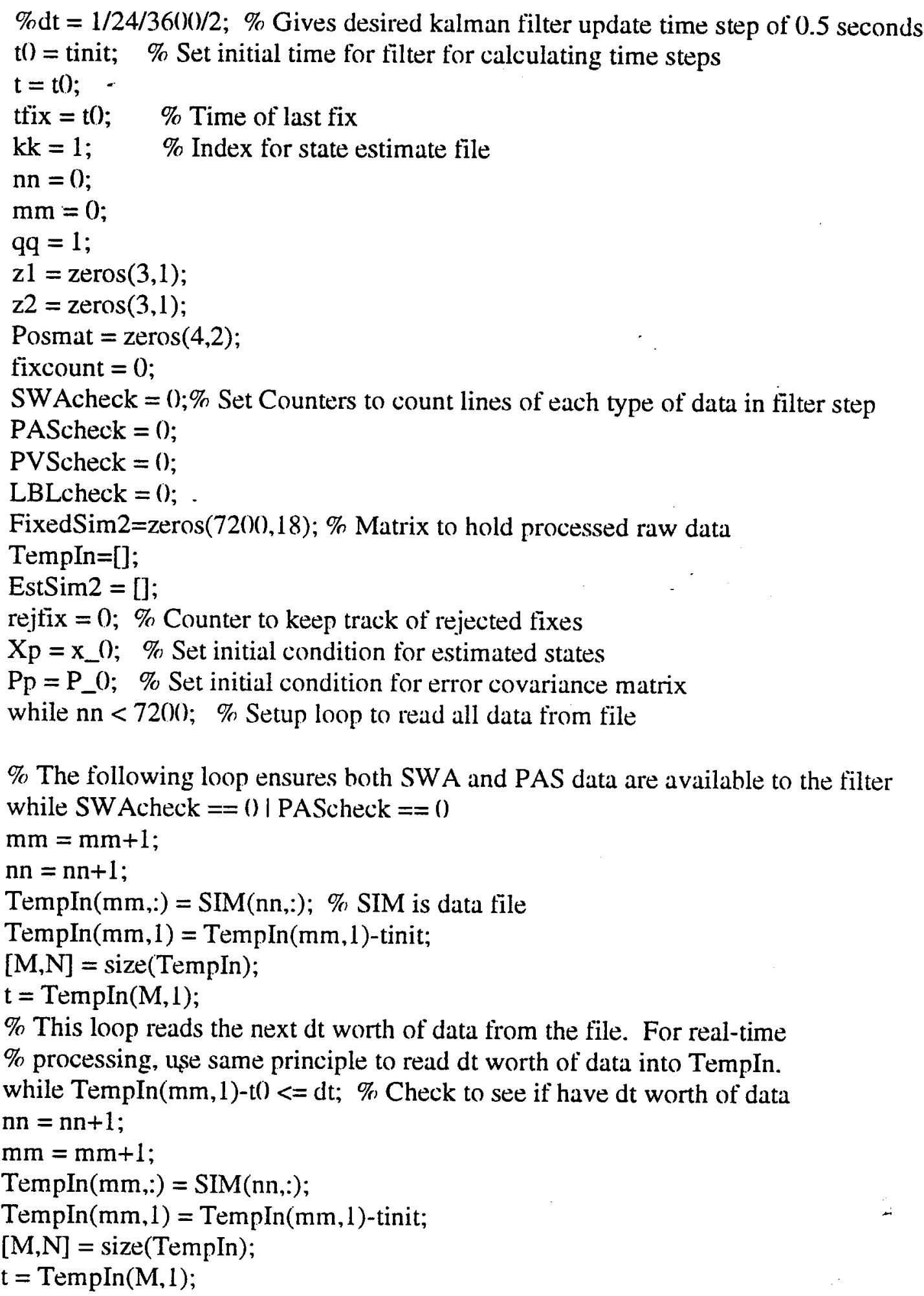


end

\% This loop checks if both SWA and PAS data is present. If the data does not $\%$ contain at least one sample of both SWA and PAS data, another dt worth will $\%$ be added prior to proceeding.

for $\mathrm{i}=\mathrm{qq}: \mathrm{M}$

if TempIn(i,2) <10631 | TempIn $(i, 2)>10633$

SWAcheck = SWAcheck +1 ;

else

TempIn(i,2:7) $=$ zeros $(1,6) ; \%$ set bogus data to zero

end

if TempIn(i,13) <10631 | TempIn $(i, 13)>10633$

PAScheck $=$ PAScheck +1 ;

$\operatorname{TempIn}(\mathrm{i}, 13)=$ zinit-TempIn(i,13);

TempIn(i,14:15) = TempIn(i,14:15). *DTOR;

if $(360-$ TempIn $(1,16))<75$ । TempIn $(1,16)<75$

if TempIn $(\mathrm{i}, 16)>270$

TempIn $(i, 16)=$ TempIn $(i, 16)-36()$;

else

end

else

end

TempIn(i,16) = TempIn $(i, 16) *$ DTOR;

if $(360$-TempIn $(1,17))<75$ । TempIn $(1,17)<75$

if $\operatorname{TempIn}(\mathrm{i}, 17)>270$

TempIn(i,17)=TempIn(i,17)-360;

else

end

else

end

TempIn $(i, 17)=$ TempIn $(i, 17) *$ DTOR;

else

TempIn(i,13:17) = zeros $(1,5) ; \%$ set bogus data to zero

end

if TempIn(i,8) <10631 | TempIn(i,8) > 10633

PVScheck = PVScheck +1 ;

else

TempIn(i,8:10) $=$ zeros $(1,3) ; \%$ set bogus data to zero

$\operatorname{TempIn}(\mathrm{i}, 18)=(0$;

end

if TempIn(i,11) <106.31 | TempIn(i,11) $>106.33$

LBLcheck $=$ LBLcheck +1 ;

TempIn(i,11) = TempIn(i,11)-xinit;

TempIn $(\mathrm{i}, 12)=$ TempIn(i,12)-yinit;

else

TempIn(i,11:12) = zeros $(1,2) ; \%$ set bogus data to zero 
end

end

$\mathrm{qq}=\mathrm{M}+\mathrm{l}$

end

FixedSim2((nn+1-M):nn+1-1,:)=TempIn;

\% Now we will provide the estimates of $x^{\wedge}(t+1 / t)$ and $P(t+1 / t)$. These are done

$\%$ now rather as the last step in the filter propagation due to the variable

$\%$ time step. Now that we know the time step, we can more accurately project

$\%$ the step estimates into the future.

$\left.\% \mathrm{DT}=(\mathrm{t}-\mathrm{t}())^{*} 24 * 36()\right) ; \%$ This is the length of the current filter time step.

$[X m, P m]=$ fullkfpred $(A, G, w, Q, X p, P p, D T)$;

$\%$ We now have enough data to run the filter. For data with more than one time $\%$ sample's worth present, the data for the whole time step is averaged.

$\%$ Also, any suspected bias is removed. The inputs are converted into the proper $\%$ units for filter use. Also, the data is checked to see if LBL and/or doppler

$\%$ data is available, which will determine which version of the filter to use.

$\%$ Therefore, the input to the kalman filter will have only one line of

\% averaged data, which will always include SWA and PAS data, and may also

\% have LBL and/or doppler data.

$\mathrm{zl}(1)=(((\operatorname{sum}($ TempIn(:,2))/SWAcheck $))-$ xacchias $) *(-\mathrm{g})+((\sin (\mathrm{Xp}(13))) * \mathrm{~g}) ;$

$\mathrm{zl}(2)=(((\operatorname{sum}($ TempIn $(:, 3)) /$ SW Acheck $))$-yaccbias $) *(-\mathrm{g})-((\sin (\mathrm{Xp}(14))) * \mathrm{~g})$;

zl(3) $\quad=\quad(((\operatorname{sum}(T e m p I n(:, 4)) / S W A c h e c k)) \text {-zacchias })^{*}(-g)$ -

$((\cos (\mathrm{Xp}(13)) * \cos (\mathrm{Xp}(14))) * \mathrm{~g})$;

$\mathrm{z} 2(1)=(((\operatorname{sum}($ TempIn $(:, 5)) / \mathrm{SWAcheck}))$-rtrias $) * \mathrm{DTOR}$;

$\mathrm{z} 2(2)=(((\operatorname{sum}($ TempIn $(:, 6)) / \mathrm{SW}$ Acheck $))$-prbias $) * \mathrm{DTOR}$;

$\mathrm{z} 2(3)=(((\operatorname{sum}($ TempIn $(:, 7)) /$ SWAcheck $))$-yrbias $) *$ DTOR;

$\% \mathrm{zl}=\mathrm{zeros}(3,1) ; \%$ Used only if not using actual accelerometer measurements

$\% \mathrm{z} 2=\mathrm{zeros}(3,1)$;

\% One other requirement is to check for position flyers. If a position update

$\%$ is bogus, it will be ignored and the filter run without it. A position update

$\%$ will be compared with the current filter estimate of position. If they differ

$\%$ by more than five times the std deviation of the position uncertainty, the

$\%$ position update will be ignored.

if LBLcheck $\sim=0$

tcheck $=(\mathrm{t}$-tfix $)$;

Pcheckx = sum(TempIn $(:, 11)) /$ LBLcheck;

Pchecky = sum(TempIn(:,12))/LBLcheck;

errest $=\operatorname{sqrt}\left(\left((\operatorname{Pcheckx}-\mathrm{Xp}(7))^{\wedge} 2\right)+(\text { Pchecky-X } \mathrm{p}(8))^{\wedge} 2\right)$;

if errest $>(10 * \operatorname{RPos}(1,1))+(0.05 *$ tcheck $)$

rejtix $=$ rejfix +1

LBLcheck $=()$;

else

tfix = ; 
end

end

$\%$ The first case is when all data is available. True heading will only be $\%$ available after five fixes have heen obtained.

\% Angular measurements have already been converted to radians using DTOR.

if LBLcheck $\sim=0$ \& PVScheck $\sim=0$

fixcount $=$ fixcount +1 ;

$\mathrm{Z}=\operatorname{zeros}(17,1)$;

$\mathrm{Z}(\mathrm{l}: 3)=\mathrm{z} 1$

$Z(4)=\operatorname{sum}($ TempIn $(:, 8)) /$ PVScheck;

$Z(5)=\operatorname{sum}($ TempIn $(:, 9)) /$ PVScheck;

$Z(6)=\operatorname{sum}($ TempIn $(:, 10)) /$ PVScheck;

$x L B L=\operatorname{sum}($ TempIn $(:, 11)) /$ LBLcheck;

$Z(7)=x L B L$;

$y L B L=\operatorname{sum}($ TempIn(:,12))/LBLcheck;

$Z(8)=y L B L ;$

$Z(9)=\operatorname{sum}($ TempIn(:,13))/PAScheck;

$Z(10: 12)=z 2$;

$Z(13)=\operatorname{sum}($ TempIn(:,14))/PAScheck;

$Z(14)=\operatorname{sum}($ TempIn $(:, 15)) /$ PAScheck;

ghdg = sum(TempIn(:,16))/PAScheck;

if ghdg<()

ghdg $=\operatorname{ghd} g+(2 * \mathrm{pi})$;

else

end

$Z(15)=$ ghdg;

mhdg = sum(TempIn(:,17))/PAScheck;

if $\mathrm{mhdg}<()$

$\mathrm{mhdg}=\mathrm{mhdg}+(2 * \mathrm{pi})$;

else

end

$\mathrm{Z}(16)=$ mhdg;

$\mathrm{R}=$ zeros $(17)$;

$\mathrm{R}(1: 3,1: 3)=\mathrm{RACC}$

$\mathrm{R}(4: 6,4: 6)=\operatorname{eye}(3){ }^{*}\left((\operatorname{sum}(\text { TempIn }(:, 18)) / \mathrm{PVScheck})^{\wedge} 2\right) ;$

$\mathrm{R}(7: 8,7: 8)=\mathrm{RPos}$;

$\mathrm{R}(9,9)=\mathrm{RDepth}$;

$R(10: 12,10: 12)=R A R$;

$R(13: 14,13: 14)=R P R$;

$\mathrm{R}(15,15)=\mathrm{RHG}$;

$\mathrm{R}(16,16)=\mathrm{RHM}$

$\mathrm{C}=\mathrm{CF}$;

\% Update the matrix containing the last four position fixes

$\operatorname{Posmat}(1,:)=\operatorname{Posmat}(2,:)$; 


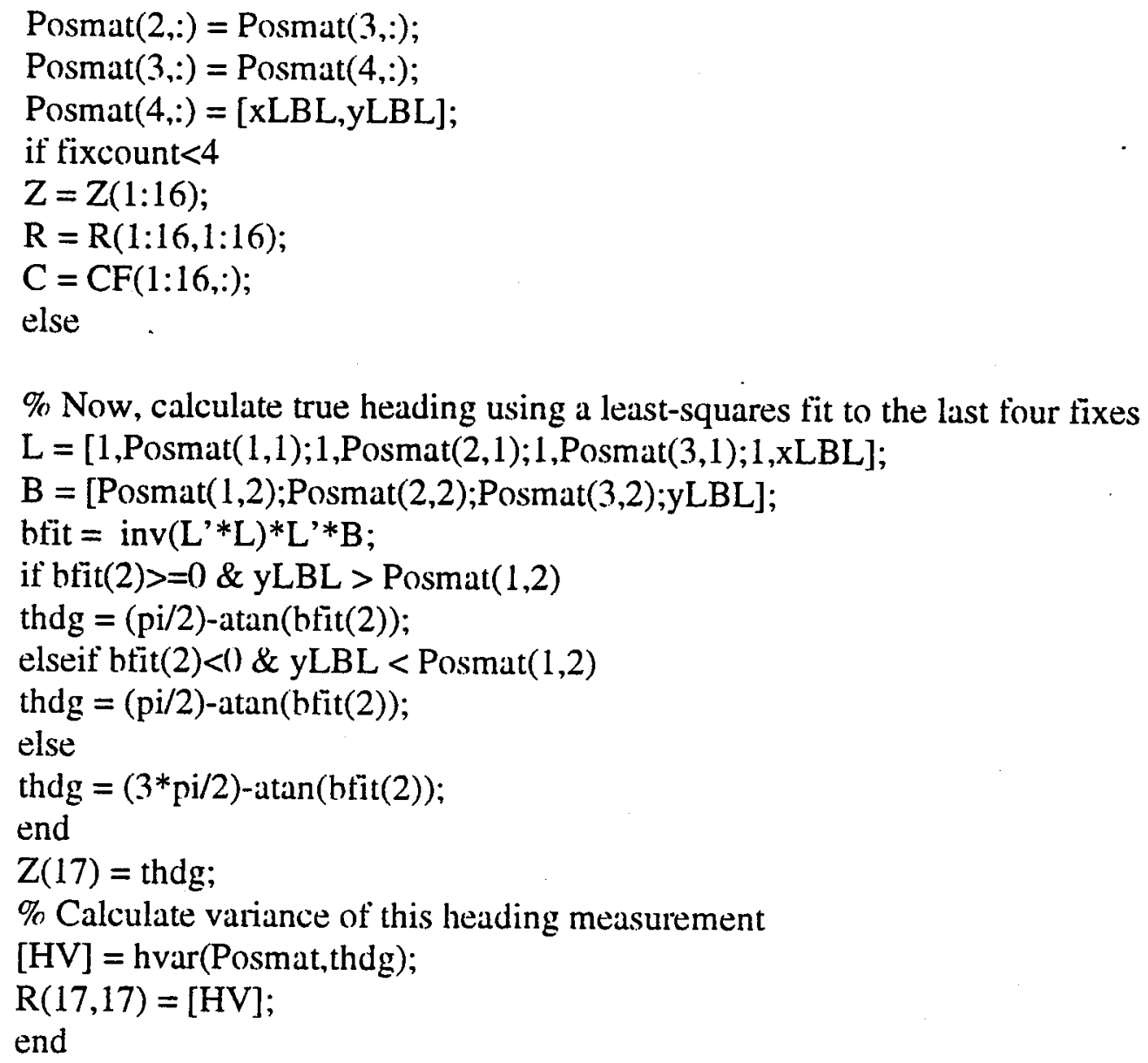


mhdg $=\operatorname{sum}($ TempIn $(:, 17)) /$ PAScheck;

if $\mathrm{mhdg}<0$

mhdg $=$ mhdg+(2*pi);

else

end

$\mathrm{Z}(13)=\mathrm{mhdg}$;

$\mathrm{R}=\operatorname{zeros}(14,14)$;

$\mathrm{R}(1: 3,1: 3)=\mathrm{RACC}$;

$\mathrm{R}(4: 5,4: 5)=\mathrm{RPos}$;

$\mathrm{R}(6,6)=\mathrm{RDepth}$;

$\mathrm{R}(7: 9,7: 9)=\mathrm{RAR}$;

$\mathrm{R}(10: 11,10: 11)=\mathrm{RPR}$;

$\mathrm{R}(12,12)=\mathrm{RHG}$;

$\mathrm{R}(13,13)=\mathrm{RHM}$;

$\mathrm{C}=\mathrm{CNV} ; \%$ Update the matrix containing the last four position tixes

$\operatorname{Posmat}(1,:)=\operatorname{Posmat}(2,:)$;

$\operatorname{Posmat}(2,:)=\operatorname{Posmat}(3,:)$;

$\operatorname{Posmat}(3,:)=\operatorname{Posmat}(4,:)$;

$\operatorname{Posmat}(4,:)=[x L B L, y L B L]$;

if fixcount $<4$

$\mathrm{Z}=\mathrm{Z}(1: 13)$

$\mathrm{R}=\mathrm{R}(1: 13,1: 13)$;

$\mathrm{C}=\mathrm{CNV}(1: 13,:)$;

else

$\%$ Calculate true heading

$\mathrm{L}=[1, \operatorname{Posmat}(1,1) ; 1, \operatorname{Posmat}(2,1) ; 1, \operatorname{Posmat}(3,1) ; 1, \mathrm{xLBL}]$;

$\mathrm{B}=[\operatorname{Posmat}(1,2) ; \operatorname{Posmat}(2,2) ; \operatorname{Posmat}(3,2) ; \mathrm{yLBL}]$;

bfit $=\operatorname{inv}\left(\mathrm{L}^{\prime} * \mathrm{~L}\right) * \mathrm{~L}{ }^{*} \mathrm{~B}$;

if bfit $(2)>=0$ \& yLBL $>$ Posmat $(1,2)$

thdg $=(\mathrm{pi} / 2)-\operatorname{atan}($ bfit (2));

elseif bfit (2)<() \& yLBL < Posmat $(1,2)$

thdg $=($ pi/2)-atan(hfit $(2))$;

else

thdg $=(3 * \mathrm{pi} / 2)-\operatorname{atan}($ bfit $(2))$;

end

$Z(14)=$ thdg;

$\%$ Calculate variance of this heading measurement

[HV] = hvar(Posmat,thdg);

$\mathrm{R}(14,14)=[\mathrm{HV}]$

end

\% The third case is when SWA, PAS, and doppler data is available, but no LBL.

elseif LBLcheck $==() \&$ PVScheck $\sim=0$

$Z=\operatorname{zeros}(14,1)$

$\mathrm{Z}(1: 3)=\mathrm{z} 1$ 


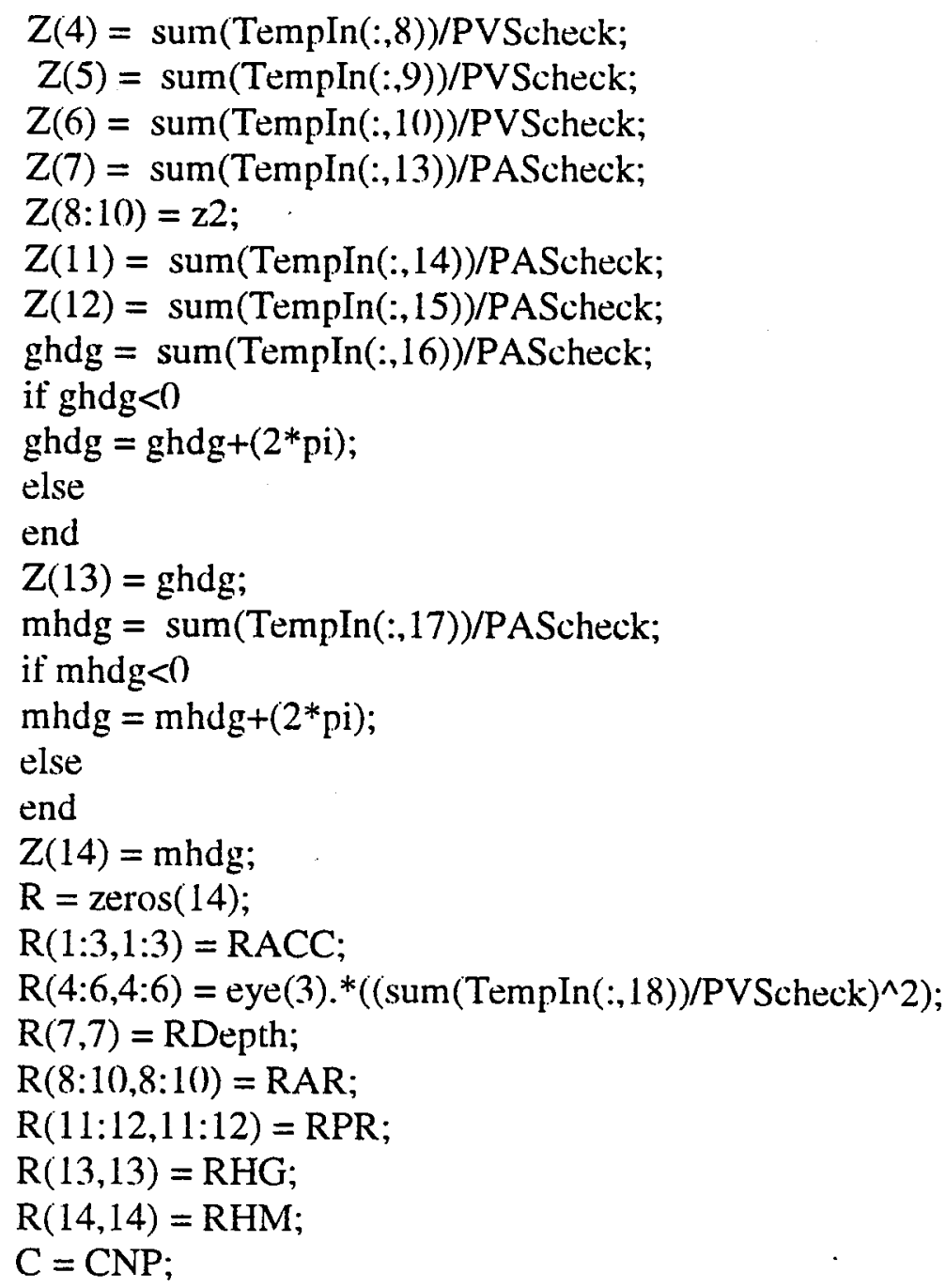

\% The last case is when only SWA and PAS data is available.

else

$\mathrm{Z}=\operatorname{zeros}(11,1)$

$\mathrm{Z}(\mathrm{l}: 3)=\mathrm{zl}$

$\mathrm{Z}(4)=\operatorname{sum}($ TempIn $(:, 13)) /$ PAScheck;

$\mathrm{Z}(5: 7)=\mathrm{z} 2$;

$Z(8)=\operatorname{sum}($ TempIn $(:, 14)) /$ PAScheck;

$\mathrm{Z}(9)=\operatorname{sum}($ TempIn $(:, 15)) /$ PAScheck;

ghdg $=\operatorname{sum}($ TempIn $(:, 16)) /$ PAScheck;

if $\operatorname{ghd} \mathrm{g}<0$

ghdg $=$ ghdg+(2*pi);

else

end

$Z(10)=$ ghdg;

mhdg = sum(TempIn $(:, 17)) /$ PAScheck;

if mhdg $<0$ 


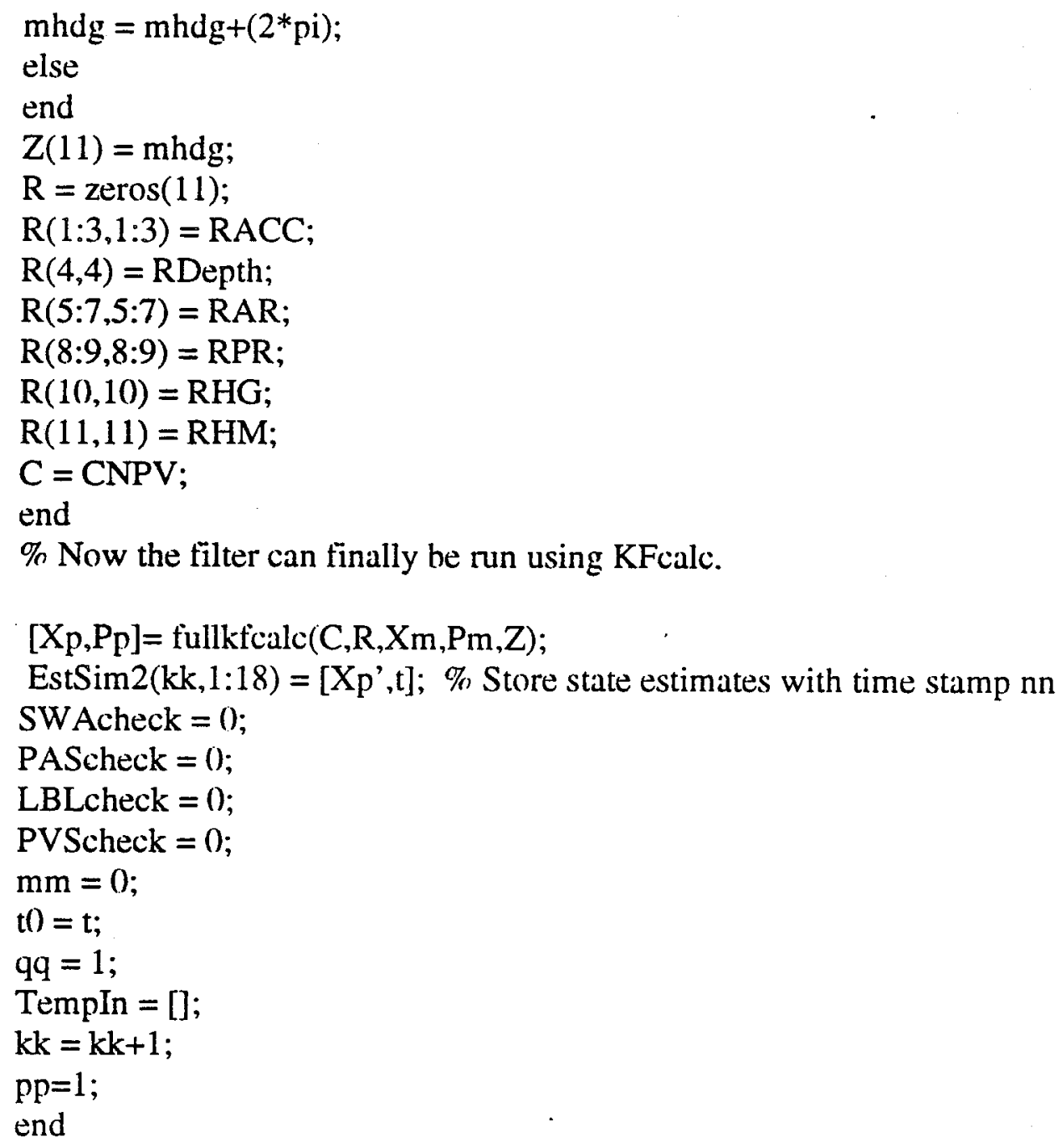




\section{KFpred}

$\%$ The function kfpred provides the estimates of $x^{\wedge}(t+1 \mid t)$ and $P(t+1 \mid t)$.

$\%$ It must be done out of the normal order for a Kalman filter since the time

$\%$ step is unknown until the next block of data is processed.

\% The format is: $[\mathrm{Xm}, \mathrm{Pm}]=\mathrm{kfpred}(\mathrm{A}, \mathrm{G}, \mathrm{U}, \mathrm{Q}, \mathrm{xcurr}, \mathrm{P}, \mathrm{t})$

$\% t$ is the time stamp for the current update step.

function $[\mathrm{Xm}, \mathrm{Pm}]=\mathrm{kfp}$ red(A,G,U,Q,xcurr,P,t)

headg $=\operatorname{xcurr}(15)$;

pitch $=x \operatorname{curr}(14)$;

roll $=\operatorname{xcurr}(13)$;

dheadg $=(2 *$ pi - headg $) ; \%$ Change axes

pcos $=\cos$ (pitch);

$\mathrm{psin}=\sin ($ pitch $)$;

$\operatorname{rcos}=\cos ($ roll $)$

rsin $=\sin ($ roll $)$;

hoos $=\cos$ (dheadg);

hsin $=\sin$ (dheadg);

$\mathrm{A}(7,4)=\operatorname{rcos} * \mathrm{hcos} ;$

$A(7,5)=p \sin * r \sin * h c o s-p c o s * h s i n ;$

$A(7,6)=p \cos *$ rsin*hcos $+p \sin * h \sin ;$

$\mathrm{A}(8,4)=\mathrm{hsin} * \mathrm{rcos} ;$

$\mathrm{A}(8,5)=p \sin *$ rsin*hsin + pcos*hcos;

$\mathrm{A}(8,6)=p \cos *$ rsin $*$ hsin - psin*hcos;

$A(9,4)=-r \sin ; A(9,5)=p \sin * \operatorname{rcos} ;$

$\mathrm{A}(9,6)=$ pcos*ros;

$[\mathrm{Phi}, \mathrm{Gamma}]=\mathrm{c} 2 \mathrm{~d}(\mathrm{~A}, \mathrm{G}, \mathrm{t})$;

$\mathrm{Xm}=\mathrm{Phi}^{*} \mathrm{xcurr}+\mathrm{Gamma}{ }^{*} \mathrm{U} ; \% \mathrm{Xm}=\mathrm{x}^{\wedge}(\mathrm{t}+\mathrm{IIt})$

$\% \mathrm{Pm}=1.0168 * \mathrm{Phi}^{*} \mathrm{P} * \mathrm{Phi}{ }^{\prime}+\mathrm{Gamma} \mathrm{Q}^{*} \mathrm{Gamma} ; \%$ Use for maneuvering vehicle

$\mathrm{Pm}=1.0\left(1{ }^{*} \mathrm{Phi}{ }^{*} \mathrm{P} * \mathrm{Phi}{ }^{*}+\mathrm{Gamma}{ }^{*} \mathrm{Q}^{*}\right.$ Gamma'; \% Use when have position data gaps or no maneuvering 


\section{FullKFcalc}

\% The function FullKFCalc implements the kalman filter for full data sets

\% The format is: $[\mathrm{Xp}, \mathrm{Pp}]=$ fullkficalc $(\mathrm{C}, \mathrm{R}, \mathrm{Xm}, \mathrm{P}, \mathrm{Z})$

$\% t$ is the time stamp for the current update step.

function $[X p, P p]=$ fullkfialc $(C, R, X m, P, Z)$

while $\mathrm{Xm}(15)<0$

$\mathrm{Xm}(15)=\mathrm{Xm}(15)+(2 * \mathrm{pi})$;

end

while $\mathrm{Xm}(15)>2 *$ pi

$\mathrm{Xm}(15)=\mathrm{Xm}(15)-(2 *$ pi $)$;

end

while $\mathrm{Xm}(16)>\mathrm{pi}$

$\mathrm{Xm}(16)=\mathrm{Xm}(16)-(2 * \mathrm{pi})$;

end

while $X m(16)<(-p i)$

$\mathrm{Xm}(16)=\mathrm{Xm}(16)+(2 * \mathrm{pi})$;

end

while $\mathrm{Xm}(17)>\mathrm{pi}$

$\mathrm{Xm}(17)=\mathrm{Xm}(17)-(2 * \mathrm{pi})$;

end

while $\mathrm{Xm}(17)<(-\mathrm{pi})$

$\mathrm{Xm}(17)=\mathrm{Xm}(17)+(2 * \mathrm{pi})$;

end

$\mathrm{Ze}=\mathrm{C}^{*} \mathrm{Xm} ; \quad$ \% $\mathrm{Ze}=\mathrm{y}^{\wedge}(\mathrm{tt}-1)$

$\mathrm{I}=\mathrm{Z}-\mathrm{Ze} ; \quad \% \mathrm{I}=\mathrm{y}(\mathrm{t})-\mathrm{y}^{\wedge}(\mathrm{tt}-\mathrm{t}-\mathrm{l})$

$\mathrm{v}=\mathrm{C}^{*} \mathrm{P}^{*} \mathrm{C}^{\prime}+\mathrm{R} ; \quad \% \mathrm{v}=$ covariance matrix of innovations

$\mathrm{k}=\mathrm{P} * \mathrm{C}^{*} * \operatorname{inv}(\mathrm{v})$;

$\mathrm{Xp}=\mathrm{Xm}+\mathrm{k}^{*} \mathrm{I} ; \quad$ \% $\mathrm{xp}=\mathrm{x}^{\wedge}(\mathrm{tlt})$

while $\mathrm{Xp}(15)<0$

$\mathrm{Xp}(15)=\mathrm{Xp}(15)+(2 * \mathrm{pi})$

end

while $\mathrm{Xp}(15)>2 * \mathrm{pi}$

$\mathrm{Xp}(15)=\mathrm{Xp}(15)-\left(2^{*} \mathrm{pi}\right)$;

end

while $\mathrm{Xp}(16)>\mathrm{pi}$

$X p(16)=X p(16)-(2 * p i)$;

end

while $\mathrm{Xp}(16)<(-\mathrm{pi})$

$\mathrm{Xp}(16)=\mathrm{Xp}(16)+\left(2^{*} \mathrm{pi}\right)$;

end

while $X p(17)>p i$

$X p(17)=X p(17)-(2 * p i)$; 
end

while $\mathrm{Xp}(17)<(-\mathrm{pi})$

$\mathrm{Xp}(17)=\mathrm{Xp}(17)+(2 * \mathrm{pi})$;

end

temp $=$ eye $(17)-\left(k^{*} \mathrm{C}\right)$;

$\mathrm{Pp}=$ temp*P*temp'+k*R*k';
$\% \mathrm{p}=\mathrm{P}(\mathrm{t} \mid \mathrm{t})$ 


\section{HVar}

$\%$ The function hvar provides the variance of the true heading $\%$ measurement.

\% The format is $[\mathrm{HV}]=$ hvar(Posmat,thdg)

function $[\mathrm{HV}]=$ hvar(Posmat, thdg)

tempvar $=0$;

Htemp $=$ zeros $(3,1)$;

for $\mathrm{i}=1: 3$;

$\mathrm{LL}=[1, \operatorname{Posmat}(\mathrm{i}, 1) ; 1, \operatorname{Posmat}(\mathrm{i}+1,1)]$;

$\mathrm{BB}=[\operatorname{Posmat}(\mathrm{i}, 2) ; \operatorname{Posmat}(\mathrm{i}+1,2)]$;

bbfï $=$ inv $\left(\mathrm{LL}^{\prime} * \mathrm{LL}\right) * \mathrm{LL}{ }^{*} * \mathrm{BB}$;

if bbfit(2)>=() \& $\mathrm{BB}(2)>\mathrm{BB}(1)$

temphdg = (pi/2)-atan(bbfit(2));

elseif bbfit $(2)<() \& B B(2)<B B(1)$

temphdg $=($ pi/2)-atan(bbiit(2));

else temphdg $=\left(3^{*}\right.$ pi/2)-atan(bbtit(2));

end

Htemp(i) = temphdg;

end

for $\mathrm{i}=1: 3$;

if Htemp(i)-thdg $>$ pi

HVar(i) $=\left((\operatorname{Htemp}(\mathrm{i})-(2 * p i))-\operatorname{thd}^{\prime}\right)^{\wedge} 2$;

elseif thdg-Htemp(i)>pi

$\mathrm{HVar}(\mathrm{i})=\left(\left(\operatorname{thdg}-\left(2^{*} \mathrm{pi}\right)\right)-\mathrm{Htemp}(\mathrm{i})\right)^{\wedge} 2$;

else

HVar(i) $=(\text { Htemp }(\mathrm{i})-\text { thdg })^{\wedge} 2$;

end

end

$\mathrm{HV}=(\operatorname{sum}(\mathrm{HVar})) / 3$; 